\title{
Berezinskii-Kosterlitz-Thouless transition and BCS-Bose crossover in the two-dimensional attractive Hubbard model
}

\author{
N. Dupuis \\ Laboratoire de Physique des Solides, CNRS UMR 8502, \\ Université Paris-Sud, 91405 Orsay, France
}

(Dated: June 7, 2004)

\begin{abstract}
We study the two-dimensional attractive Hubbard model using the mapping onto the half-filled repulsive Hubbard model in a uniform magnetic field coupled to the fermion spins. The low-energy effective action for charge and pairing fluctuations is obtained in the hydrodynamic regime. We recover the action of a Bose superfluid where half the fermion density is identified as the conjugate variable of the phase of the superconducting order parameter. By integrating out charge fluctuations, we obtain a phase-only action. In the zero-temperature superconducting state, this action describes a collective phase mode smoothly evolving from the Anderson-Bogoliubov mode at weak coupling to the Bogoliubov mode of a Bose superfluid at strong coupling. At finite temperature, the phase-only action can be used to extract an effective XY model and thus obtain the Berezinskii-KosterlitzThouless (BKT) phase transition temperature. We also identify a renormalized classical regime of superconducting fluctuations above the BKT phase transition, and a regime of incoherent pairs at higher temperature. Special care is devoted to the nearly half-filled case where the symmetry of the order parameter is enlarged to $\mathrm{SO}(3)$ due to strong $\mathbf{q}=(\pi, \pi)$ charge fluctuations. The low-energy effective action is then an $\mathrm{SO}(3)$ non-linear sigma model with a (symmetry breaking) magnetic field proportional to the doping. In the strong-coupling limit, the attractive Hubbard model can be mapped onto the Heisenberg model in a magnetic field, which reduces to the quantum XY model (except for a weak magnetic field, i.e. in the low-density limit of the attractive model). In the low-density limit, the Heisenberg model allows to recover the action of a Bose superfluid, including the $(\nabla \rho)^{2}$ term (with $\rho$ the density), and in turn the Gross-Pitaevskii equation.

PACS numbers: 71.10.Fd, 74.20.Fg, 05.30.Jp
\end{abstract}

\section{INTRODUCTION}

Many superconducting systems such as high- $T_{c}$ superconductors, organic conductors, heavy fermions systems, as well as ultracold atomic Fermi gases cannot be understood within the BCS theory. For instance, in high- $T_{c}$ superconductors, the low dimensionality reinforces the role of phase fluctuations. ${ }^{1.2}$ The short coherence length in these systems also suggests that they might be in an intermediate regime between the weak-coupling BCS limit of superfluid fermions and the strong-coupling limit of condensed composite bosons ${ }^{3.4}$ In ultracold atomic Fermi gases that are now experimentally available, it appears possible to monitor the evolution from the BCS to the Bose limit $\underline{\underline{5}}$

A superconducting system at low temperature is conveniently described by a low-energy effective action written in terms of a few relevant (bosonic) variables. The minimum description requires to consider the phase of the superconducting order parameter, but other variables such as the amplitude of the order parameter or the charge density may also be included. The effective action (or the corresponding equations of motion) is sufficient to describe macroscopic quantum phenomena such as the Meissner effect, the flux quantization, the Josephson effect, or the vortex dynamics. ${ }^{6.7}$ In a Bose superfluid, the low-energy effective action leads to the Gross-Pitaevskii equation, ${ }^{8.9}$ i.e. a non-linear Schrödinger equation for the complex (superfluid) order parameter $\Psi$ where $|\Psi|^{2}=\rho$ is the condensate density. In a Fermi system, there is in general no simple relation between the amplitude of the superconducting order parameter and the density. In the strong-coupling limit, where fermions form tightly bound pairs which behave as bosons, we expect the Gross-Pitaevskii equation to hold. Moreover, since Fermi and Bose superfluids should behave similarly in many respects, a Fermi superfluid should be described by a non-linear Schrödinger equation similar to the GrossPitaevskii equation even in the weak-coupling limit. ${ }^{6}$ This is the conclusion reached by previous works, 10.11 .12 although the "wavefunction" in this case is not the superfluid order parameter (except in the strong-coupling limit).

The low-energy effective action provides a convenient framework to discuss the BCS-Bose crossover between the weak-coupling BCS limit and the Bose limit of preformed pairs 12.13 .14 .15 .16 .17 .18 In two dimensions (2D), there is an additional motivation to introduce an effective action written in terms of the phase of the order parameter. The Berezinskii-Kosterlitz-Thouless (BKT) phase transition ${ }^{19.20}$ which takes place in a 2D Fermi superfluid is clearly out of reach of fermionic approximations based on diagram resummations like the $T$-matrix approximation ${ }^{3}$

While the 2D attractive Hubbard model is not an appropriate microscopic model for most superconducting systems of interest, it can be used to understand a number of general issues relevant to many cases. The main characteristics of this model are well-known.21.22 Away from half-filling, there is a BKT phase transition to a 
low-temperature superconducting phase. Long-range order sets in at zero temperature and breaks $\mathrm{SO}(2)$ symmetry. At half-filling, $\mathbf{q}=0$ pairing and $\mathbf{q}=(\pi, \pi)$ charge fluctuations combine to form an order parameter with $\mathrm{SO}(3)$ symmetry. The superconducting transition then occurs at zero temperature and breaks $\mathrm{SO}(3)$ symmetry. In the weak-coupling limit, superconductivity is due to strongly overlapping Cooper pairs, and Bogoliubov quasiparticle excitations dominate the low-energy physics. In the strong-coupling limit, fermions form tightly bound pairs that Bose condense at low temperature thus giving rise to superfluidity. Pair-breaking excitations are not possible at low energy and the thermodynamics is controlled by (collective) phase fluctuations.

In this paper, we derive the low-energy effective action for charge and pairing fluctuations in the 2D attractive Hubbard model on a square lattice. We discuss the BCSBose crossover and the phase diagram. We use the mapping of the attractive model onto the repulsive half-filled model in a magnetic field which couples to the fermion spins. In this mapping, the charge and pairing fields transform into the three components of the spin-density field. At half-filling, the magnetic field vanishes in the repulsive model. This case has been studied in detail in Refs. 2324 . At zero temperature, the system evolves from a Slater to a Mott-Heisenberg antiferromagnet as the interaction strength (i.e. the on-site Coulomb repulsion) increases. Because of the $\mathrm{SO}(3)$ symmetry of the order parameter, the Néel temperature vanishes in agreement with the Mermin-Wagner ${ }^{25}$ theorem. At finite temperature, the system is in a renormalized classical ( $\mathrm{RC}$ ) regime with an exponentially large antiferromagnetic correlation length. Away from half-filling, the magnetic field reduces the symmetry to $\mathrm{SO}(2)$ so that a BKT phase transition occurs at finite temperature. In the attractive model, this corresponds to the suppression of $\mathbf{q}=(\pi, \pi)$ charge fluctuations at low energy. The main advantage of studying the repulsive model is that the $\mathrm{SO}(3)$ symmetry of the order parameter at or near half-filling can be easily handled.

In Sec. II we map the attractive model onto the repulsive one by a canonical particle-hole transformation ${ }^{26}$ and then obtain the effective action for the spin fluctuations in the presence of a finite magnetic field (i.e. away from half-filling in the attractive model). Collective bosonic fields are introduced by means of a Hubbard-Stratonovich decoupling of the Hubbard interaction. This crucial step in our approach differs from the usual decoupling in two respects. First, we write the interaction in an explicit $\mathrm{SO}(3)$ spin-rotation form by introducing a unit vector $\Omega_{\mathbf{r}}$ at each site and time. ${ }^{23,24,27,28}$ This allows to recover the Hartree-Fock (HF) theory at the saddle-point level, while maintaining $\mathrm{SO}(3)$ spinrotation symmetry (in the absence of the magnetic field). Second, we introduce two auxiliary real fields. One is simply the Hubbard-Stratonovich field $m_{\mathbf{r}}^{\mathrm{HS}}$ for the amplitude of the spin density at site $\mathbf{r}$. The other one, $m_{\mathbf{r}}$, is directly connected to the actual amplitude of the spin density. ${ }^{29}$ While these two fields are proportional at the saddle-point (i.e. Hartree-Fock) level, they differ when fluctuations are taken into account. In the weak- and strong-coupling limits, $\frac{m_{\mathrm{r}}}{2} \boldsymbol{\Omega}_{\mathrm{r}}$ can be identified with the spin density, which allows a direct interpretation of the low-energy action $S[m, \boldsymbol{\Omega}]$ in terms of physical quantities. Because of the magnetic field, the spin component along the field, $S^{z}$, takes a finite value and its fluctuations are small at low energy. Since spin amplitude fluctuations are also small, the important fluctuations correspond to rotations around the magnetic field axis. We derive the effective action $S[m, \boldsymbol{\Omega}]$ in this case. $S[m, \boldsymbol{\Omega}]$ takes a simple form in the weak-coupling (Slater) and strong-coupling (Mott-Heisenberg) limits. These two limits differ in the role of the Berry phase term.

In Sec. IIC we deduce the effective action $S[\rho, \Delta]$ of the charge $(\rho)$ and pairing $\left(\Delta=|\Delta| e^{i \Theta}\right)$ fluctuations in the attractive model. By integrating out amplitude fluctuations $(|\Delta|)$, we recover the action $S[\rho, \Theta]$ of a Bose superfluid where half the fermion density is identified as the conjugate variable of the phase $\Theta$ of the superconducting order parameter. This action is parametrized by the mass $m_{b}$ of the "bosons" and the amplitude $g$ of the repulsive interaction between "bosons". $m_{b}$ and $g$ are computed as a function of particle density and interaction strength. We then analyze in more detail the weak (BCS) and strong (Bose) coupling limits. In the BCS limit, we find that the action is a function of charge and phase fluctuations only, since amplitude fluctuations of the superconducting order parameter decouple. In the strong-coupling limit, the amplitude of the superconducting order parameter and the fermion density $\rho$ satisfy the relation $\left|\Delta_{\mathbf{r}}\right|=\frac{1}{2} \sqrt{\rho_{\mathbf{r}}\left(2-\rho_{\mathbf{r}}\right)}$. The Bose superfluid action $S[\rho, \Theta] \equiv S[|\Delta|, \Theta]$ then entirely describes the dynamics of the superconducting order parameter $\Delta$. In Sec. IID we derive the phase-only action $S[\Theta]$ by integrating out charge fluctuations. $S[\Theta]$ corresponds to an $\mathrm{O}(2)$ sigma model with an additional term proportional to the first-order time derivative of $\Theta$. The phase stiffness and the velocity of the $\mathrm{O}(2)$ sigma model are obtained as a function of particle density and interaction strength. At zero temperature, superconducting long-range order gives rise to a gapless (Goldstone) phase mode smoothly evolving from the Anderson-Bogoliubov mode $e^{30}$ at weak coupling to the Bogoliubov mode ${ }^{31}$ of a Bose superfluid at strong coupling. In Sec. IIE we show how we can extract from the phase-only action an effective (classical) $\mathrm{XY}$ model whose phase stiffness is a function of density, interaction strength and temperature. This allows us to determine the value of the BKT phase transition temperature as a function of density and interaction strength. The XY model also yields an estimate of the crossover temperature $T_{X}$ below which the system enters a RC regime of phase fluctuations. At higher temperature, for $T_{X} \leq T \leq T_{\text {pair }}$, there is a regime of incoherent pairs [Cooper (local) pairs at weak (strong) coupling] with no superconducting short-range order. $T_{\text {pair }}$ is estimated from the $\mathrm{HF}$ transition temperature. 
In the vicinity of half-filling, the analysis of Sec. II is not sufficient since $\mathbf{q}=(\pi, \pi)$ charge fluctuations (in the attractive model) are not considered. For a weak magnetic field (in the repulsive model), there are strong fluctuations of $S^{z}$, and the analysis of Sec. III breaks down. This case is dealt with in Sec. III We show that the dynamics of spin fluctuations in the repulsive model is governed by an $\mathrm{SO}(3)$ non-linear-sigma model with a (symmetry-breaking) magnetic field proportional to the doping. The magnetic field defines a characteristic temperature $T_{\mathrm{SO}(3 \rightarrow 2)}$ above which the $\mathrm{SO}(3)$ spin-rotation symmetry is restored. Below $T_{\mathrm{SO}(3 \rightarrow 2)}$, the system enters a $\mathrm{RC}$ regime of spin fluctuations with $\mathrm{SO}(2)$ symmetry. The global phase diagram, as a function of density, interaction strength and temperature, is discussed in Sec. IIF] [For clarity, we discuss the phase diagram at the end of Sec. II and postpone the technical analysis of the $\mathrm{SO}(3) \rightarrow \mathrm{SO}(2)$ crossover near half-filling to Sec. III]

In Sec. IV we consider the strong-coupling limit in more detail. First we show that the repulsive Hubbard model reduces to the Heisenberg model to leading order in $1 / U$. This allows to obtain the collective modes beyond the long-wavelength approximation. We then show that except for a strong magnetic field (i.e. in the low density limit of the attractive model), the Heisenberg model in a magnetic field reduces to the quantum XY model. In the low-density limit, we recover from the Heisenberg model the usual action of a Bose superfluid (for bosons of mass $1 / J=U / 4 t^{2}$ and density $\rho / 2$ ), including the terms proportional to $\left(\nabla \rho_{\mathbf{r}}\right)^{2}$ that were omitted in Sec. II The classical equation of motion derived from this action is nothing but the Gross-Pitaevskii equation. We therefore obtain a correspondence between the Gross-Pitaevskii equation in the attractive model and the semiclassical spin dynamics in the repulsive model.

To our knowledge, there is no systematic study of the $2 \mathrm{D}$ repulsive Hubbard model in a magnetic field. When translated in the language of the attractive model, our results reproduce, in a unique framework, a number of previously known results. We also obtain new results, in particular regarding the BCS-Bose crossover and the phase diagram.

\section{AWAY FROM HALF-FILLING}

The attractive Hubbard model on a square lattice is defined by the Hamiltonian

$$
H=-\sum_{\mathbf{r}} c_{\mathbf{r}}^{\dagger}(\hat{t}+\mu) c_{\mathbf{r}}-U \sum_{\mathbf{r}} n_{\mathbf{r} \uparrow} n_{\mathbf{r} \downarrow}
$$

where $\hat{t}$ is the nearest-neighbor hopping operator:

$$
\hat{t} c_{\mathbf{r}}=t\left(c_{\mathbf{r}+\hat{\mathbf{x}}}+c_{\mathbf{r}-\hat{\mathbf{x}}}+c_{\mathbf{r}+\hat{\mathbf{y}}}+c_{\mathbf{r}-\hat{\mathbf{y}}}\right) .
$$

$\hat{\mathbf{x}}$ and $\hat{\mathbf{y}}$ denote unit vectors along the $x$ and $y$ axis. With the notation of Eq. (1), $-U$ is the on-site interaction with
$U \geq 0$ in the attractive case. The operator $c_{\mathbf{r} \sigma}^{\dagger}\left(c_{\mathbf{r} \sigma}\right)$ creates (annihilates) a fermion of spin $\sigma$ at the lattice site $\mathbf{r}, c_{\mathbf{r}}=\left(c_{\mathbf{r} \uparrow}, c_{\mathbf{r} \downarrow}\right)^{T}$, and $n_{\mathbf{r} \sigma}=c_{\mathbf{r} \sigma}^{\dagger} c_{\mathbf{r} \sigma}$. At half-filling, particle-hole symmetry implies that the chemical potential $\mu$ equals $-U / 2$. In the following, we will consider only hole doping so that $\mu \leq-U / 2$. We take the lattice spacing equal to unity and $\hbar=k_{B}=1$.

Under the canonical particle-hole transformation ${ }^{26}$

$$
c_{\mathbf{r} \downarrow} \rightarrow(-1)^{\mathbf{r}} c_{\mathbf{r} \downarrow}^{\dagger}, \quad c_{\mathbf{r} \downarrow}^{\dagger} \rightarrow(-1)^{\mathbf{r}} c_{\mathbf{r} \downarrow}
$$

the Hamiltonian becomes (omitting a constant term)

$$
H=-\sum_{\mathbf{r}} c_{\mathbf{r}}^{\dagger}\left[\hat{t}+\left(\mu+\frac{U}{2}\right) \sigma^{z}+\frac{U}{2}\right] c_{\mathbf{r}}+U \sum_{\mathbf{r}} n_{\mathbf{r} \uparrow} n_{\mathbf{r} \downarrow},
$$

where $\left(\sigma^{x}, \sigma^{y}, \sigma^{z}\right)$ denotes the Pauli matrices. The transformed Hamiltonian (4) corresponds to the repulsive halffilled Hubbard model in a magnetic field

$$
h_{0} \equiv \mu+\frac{U}{2}
$$

along the $z$ axis coupled to the fermion spins. In the attractive model, the chemical potential $\mu$ is fixed by the condition $\left\langle c_{\mathbf{r}}^{\dagger} c_{\mathbf{r}}\right\rangle=\rho_{0}=1-x$ where $\rho_{0}$ is the mean density and $x$ the doping. Under the particle-hole transformation (3), $c_{\mathbf{r}}^{\dagger} c_{\mathbf{r}} \rightarrow c_{\mathbf{r}}^{\dagger} \sigma^{z} c_{\mathbf{r}}+1$. In the repulsive model, the magnetic field $h_{0}$ is then determined by

$$
\left\langle c_{\mathbf{r}}^{\dagger} \sigma^{z} c_{\mathbf{r}}\right\rangle=-x
$$

The charge-density and pairing operators transform as

$$
\begin{gathered}
\rho_{\mathbf{r}}=c_{\mathbf{r}}^{\dagger} c_{\mathbf{r}} \rightarrow\left(2 S_{\mathbf{r}}^{z}+1\right), \\
\Delta_{\mathbf{r}}=c_{\mathbf{r} \downarrow} c_{\mathbf{r} \uparrow} \rightarrow(-1)^{\mathbf{r}} S_{\mathbf{r}}^{-}, \\
\Delta_{\mathbf{r}}^{\dagger}=c_{\mathbf{r} \uparrow}^{\dagger} c_{\mathbf{r} \downarrow}^{\dagger} \rightarrow(-1)^{\mathbf{r}} S_{\mathbf{r}}^{+},
\end{gathered}
$$

where $S_{\mathbf{r}}^{\nu}=c_{\mathbf{r}}^{\dagger} \frac{\sigma^{\nu}}{2} c_{\mathbf{r}}(\nu=x, y, z)$ and $S_{\mathbf{r}}^{ \pm}=S_{\mathbf{r}}^{x} \pm i S_{\mathbf{r}}^{y}$. In the repulsive model, spin fluctuations are clearly the collective (bosonic) fluctuations of interest. Accordingly, in the attractive model, both pairing and charge fluctuations should be considered on equal footing. One of the motivations to study the repulsive model is that one has to consider only the particle-hole channel. On the contrary, a direct study of the attractive model would require to consider both the particle-hole and particle-particle channels in order to take into account the charge and pairing fluctuations. The simultaneous introduction of auxiliary Hubbard-Stratonovich fields in these two channels is not without problem if one requires the saddlepoint approximation to recover the HF (or mean-field) theory ${ }^{12}$ Furthermore, in the attractive model at halffilling, $\mathbf{q}=0$ pairing and $\mathbf{q}=(\pi, \pi)$ charge fluctuations combine to form an order parameter with $\mathrm{SO}(3)$ symmetry. The SO(3) symmetry of the order parameter is much more easily handled in the repulsive model, where the distance from half-filling determines the (symmetrybreaking) magnetic field $h_{0}$ (see Sec. III). 
We can write the partition function of the repulsive model as a path integral over Grassmann fields $c_{\mathbf{r} \sigma}^{*}, c_{\mathbf{r} \sigma}$, with the action

$$
S=\int_{0}^{\beta} d \tau\left\{\sum_{\mathbf{r}} c_{\mathbf{r}}^{\dagger} \partial_{\tau} c_{\mathbf{r}}+H\left[c^{\dagger}, c\right]\right\}
$$

where $\tau$ is an imaginary time and $\beta=1 / T$ the inverse temperature. $H\left[c^{\dagger}, c\right]$ is obtained from the Hamiltonian (4) by replacing the operators by the corresponding Grassmann fields. We now introduce auxiliary bosonic fields for the collective spin fluctuations in a way that fulfills the three following requirements: i) the HF (or mean-field) approximation is recovered from a saddlepoint approximation; ii) the $\mathrm{SO}(2)$ (or $\mathrm{SO}(3)$ if $h_{0}=0$ ) spin-rotation symmetry is maintained; iii) the auxiliary fields correspond to the spin-density field $\mathbf{S}_{\mathbf{r}}=c_{\mathbf{r}}^{\dagger} \frac{\sigma}{2} c_{\mathbf{r}}$ not only at the saddle-point level, but also when fluctuations are taken into account $\left[\boldsymbol{\sigma}=\left(\sigma^{x}, \sigma^{y}, \sigma^{z}\right)\right]$.

We start from the identity 23.24 .27

$$
n_{\mathbf{r} \uparrow} n_{\mathbf{r} \downarrow}=\frac{1}{4}\left[\left(c_{\mathbf{r}}^{\dagger} c_{\mathbf{r}}\right)^{2}-\left(c_{\mathbf{r}}^{\dagger} \boldsymbol{\sigma} \cdot \boldsymbol{\Omega}_{\mathbf{r}} c_{\mathbf{r}}\right)^{2}\right],
$$

where $\boldsymbol{\Omega}_{\mathbf{r}}$ is an arbitrary site- and time-dependent unit vector. $\boldsymbol{\Omega}_{\mathbf{r}}$ is defined by its polar and azimuthal angles $\theta_{\mathbf{r}}, \varphi_{\mathbf{r}}$. Spin-rotation invariance is made explicit by performing an angular integration over $\boldsymbol{\Omega}_{\mathbf{r}}$ at each site and time (with a measure normalized to unity). The charge term $\left(c_{\mathbf{r}}^{\dagger} c_{\mathbf{r}}\right)^{2}$ is decoupled by means of an auxiliary (real) field $\Delta_{c \mathbf{r}}$. In order to decouple the spin term $\left(c_{\mathbf{r}}^{\dagger} \boldsymbol{\sigma} \cdot \boldsymbol{\Omega}_{\mathbf{r}} c_{\mathbf{r}}\right)^{2}$, we introduce in the path integral the unit factor

$$
\begin{aligned}
1 & =\int \mathcal{D}[m] \prod_{\mathbf{r}, \tau} \delta\left(m_{\mathbf{r}}-c_{\mathbf{r}}^{\dagger} \boldsymbol{\sigma} \cdot \boldsymbol{\Omega}_{\mathbf{r}} c_{\mathbf{r}}\right) \\
& =\int \mathcal{D}\left[m, m^{\mathrm{HS}}\right] e^{-\int_{0}^{\beta} d \tau \sum_{\mathbf{r}} i m_{\mathbf{r}}^{\mathrm{HS}}\left(m_{\mathbf{r}}-c_{\mathbf{r}}^{\dagger} \boldsymbol{\sigma} \cdot \boldsymbol{\Omega}_{\mathbf{r}} c_{\mathbf{r}}\right)},
\end{aligned}
$$

where $m_{\mathbf{r}}^{\mathrm{HS}}$ is a Lagrange multiplier field which imposes the constraint $m_{\mathbf{r}}=2 \mathbf{S}_{\mathbf{r}} \cdot \boldsymbol{\Omega}_{\mathbf{r}}$. Both $m$ and $m^{\mathrm{HS}}$ are real fields. Note that integrating out the $m_{\mathbf{r}}$ field [see Eqs. (1112) below], one obtains the action $S\left[c^{\dagger}, c, \Delta_{c}, m^{\mathrm{HS}}\right]$ where $\Delta_{c \mathbf{r}}$ and $m^{\mathrm{HS}}$ are the HubbardStratonovich fields which decouple the interaction term (9). In general, $\frac{m_{\mathrm{r}}}{2} \boldsymbol{\Omega}_{\mathrm{r}}$ cannot be directly identified with the spin density $\mathbf{S}_{\mathbf{r}}$, but the identification turns out to be correct in the hydrodynamic regime both at weak $(U \ll 4 t)$ and strong $(U \gg 4 t)$ coupling. with

Using (9) and (10), we write the action as $S=S_{0}+S_{\text {int }}$

$$
\begin{aligned}
S_{0}= & \int_{0}^{\beta} d \tau \sum_{\mathbf{r}} c_{\mathbf{r}}^{\dagger}\left(\partial_{\tau}-\hat{t}-h_{0} \sigma^{z}-\frac{U}{2}\right) c_{\mathbf{r}}, \\
S_{\mathrm{int}}= & \int_{0}^{\beta} d \tau \sum_{\mathbf{r}}\left[\frac{\Delta_{\mathbf{r}}^{2}}{U}-\frac{U}{4} m_{\mathbf{r}}^{2}+i m_{\mathbf{r}} m_{\mathbf{r}}^{\mathrm{HS}}\right. \\
& \left.-c_{\mathbf{r}}^{\dagger}\left(i \Delta_{c \mathbf{r}}+i m_{\mathbf{r}}^{\mathrm{HS}} \boldsymbol{\sigma} \cdot \boldsymbol{\Omega}_{\mathbf{r}}\right) c_{\mathbf{r}}\right] .
\end{aligned}
$$

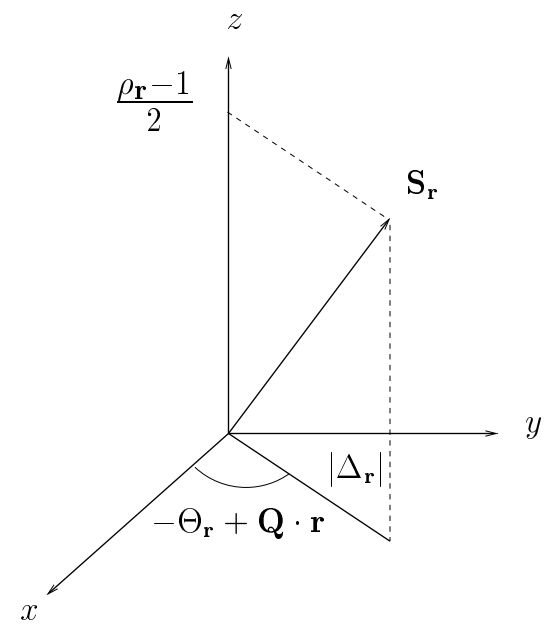

FIG. 1: Correspondence between the spin density $\mathbf{S}_{\mathbf{r}}$ in the repulsive model, and the charge density $\rho_{\mathbf{r}}$ and superconducting field $\Delta_{\mathbf{r}}=\left|\Delta_{\mathbf{r}}\right| e^{i \Theta_{\mathbf{r}}}$ in the attractive model [see Eqs. (7)].

In the following, we shall consider the charge field $\Delta_{c r}$ only at the saddle-point level, i.e. $\Delta_{c \mathbf{r}}=i(U / 2)\left\langle c_{\mathbf{r}}^{\dagger} c_{\mathbf{r}}\right\rangle=$ $i U / 2$. The term $-i c_{\mathbf{r}}^{\dagger} \Delta_{c r} c_{\mathbf{r}}$ in (12) cancels the chemical potential term $-(U / 2) c_{\mathbf{r}}^{\dagger} c_{\mathbf{r}}$ in (11).

Eqs. (1112) are the starting point of our analysis. In Sec.IIA we show that the HF theory is recovered from a saddle-point approximation over the auxiliary fields $m_{\mathbf{r}}$, $m_{\mathbf{r}}^{\mathrm{HS}}$ and $\boldsymbol{\Omega}_{\mathbf{r}}$. In the following sections, we go beyond the HF theory and derive a low-energy effective action $S[m, \boldsymbol{\Omega}]$ for spin fluctuations, and deduce the effective action $S[\rho, \Delta]$ of charge and pairing fluctuations in the attractive model.

\section{A. Hartree-Fock theory}

In the presence of the uniform magnetic field along the $z$ axis, we expect the ground state to exhibit $\mathrm{AF}$ order in the $(x, y)$ plane and a ferromagnetic order along the $z$ axis. We therefore consider a static saddle-point approximation with $m_{\mathbf{r}}=m_{0}, m_{\mathbf{r}}^{\mathrm{HS}}=m_{0}^{\mathrm{HS}}$, and a classical configuration of the unit vector field $\boldsymbol{\Omega}_{\mathrm{r}}$ given by

$$
\boldsymbol{\Omega}_{\mathbf{r}}^{\mathrm{cl}}=(-1)^{\mathbf{r}} \sin \theta_{0} \hat{\mathbf{x}}+\cos \theta_{0} \hat{\mathbf{z}} .
$$

The HF action then reads

$$
\begin{aligned}
S_{\mathrm{HF}}= & \beta N\left(-\frac{U}{4} m_{0}^{2}+i m_{0} m_{0}^{\mathrm{HS}}\right) \\
& +\int_{0}^{\beta} d \tau \sum_{\mathbf{r}} c_{\mathbf{r}}^{\dagger}\left[\partial_{\tau}-\hat{t}-h \sigma^{z}-\Delta_{0}^{\mathrm{HS}}(-1)^{\mathbf{r}} \sigma^{x}\right] c_{\mathbf{r}},
\end{aligned}
$$

where $N$ is the total number of lattice sites, and

$$
\begin{aligned}
\Delta_{0}^{\mathrm{HS}} & =i m_{0}^{\mathrm{HS}} \sin \theta_{0}, \\
h & =h_{0}+i m_{0}^{\mathrm{HS}} \cos \theta_{0} .
\end{aligned}
$$


The HF action is quadratic and can be easily diagonalized. The single-particle Green's functions are given by

$$
\begin{aligned}
G_{\sigma}(\mathbf{k}, i \omega) & =-\left\langle c_{\sigma}(\mathbf{k}, i \omega) c_{\sigma}^{*}(\mathbf{k}, i \omega)\right\rangle \\
& =\frac{-i \omega-\epsilon_{\mathbf{k} \sigma}}{\omega^{2}+E_{\mathbf{k} \sigma}^{2}}, \\
F_{\sigma}(\mathbf{k}, i \omega) & =-\left\langle c_{\sigma}(\mathbf{k}, i \omega) c_{\bar{\sigma}}^{*}(\mathbf{k}+\mathbf{Q}, i \omega)\right\rangle \\
& =\frac{\Delta_{0}^{\mathrm{HS}}}{\omega^{2}+E_{\mathbf{k} \sigma}^{2}},
\end{aligned}
$$

where

$$
\begin{aligned}
\epsilon_{\mathbf{k} \sigma} & =\epsilon_{\mathbf{k}}-\sigma h, \\
E_{\mathbf{k} \sigma} & =\sqrt{\epsilon_{\mathbf{k} \sigma}^{2}+\Delta_{0}^{\mathrm{HS}^{2}}} .
\end{aligned}
$$

$\epsilon_{\mathbf{k}}=-2 t\left(\cos k_{x}+\cos k_{y}\right)$ is the energy of the free fermions on the square lattice. $\bar{\sigma}=-\sigma, \mathbf{Q}=(\pi, \pi)$, and $\omega=\pi T(2 n+1)$ ( $n$ integer) is a fermionic Matsubara frequency. $c_{\sigma}(\mathbf{k}, i \omega)$ is the Fourier transformed field of $c_{\mathbf{r} \sigma}$.

The saddle-point equations are obtained from $\partial Z_{\mathrm{HF}} / \partial m_{0}=\partial Z_{\mathrm{HF}} / \partial \Delta_{0}^{\mathrm{HS}}=\partial Z_{\mathrm{HF}} / \partial h=0$, where $Z_{\mathrm{HF}}$ is the partition function in the HF approximation:

$$
\begin{aligned}
m_{0} & =\frac{2}{U} i m_{0}^{\mathrm{HS}}, \\
\Delta_{0} & \equiv \frac{\Delta_{0}^{\mathrm{HS}}}{U} \\
& =\frac{m_{0}}{2} \sin \theta_{0} \\
& =\frac{(-1)^{\mathbf{r}}}{2}\left\langle c_{\mathbf{r}}^{\dagger} \sigma^{x} c_{\mathbf{r}}\right\rangle, \\
m_{0} \cos \theta_{0} & =\frac{2}{U}\left(h-h_{0}\right) \\
& =\left\langle c_{\mathbf{r}}^{\dagger} \sigma^{z} c_{\mathbf{r}}\right\rangle .
\end{aligned}
$$

Eq. (6) then implies

$$
\begin{aligned}
m_{0} \cos \theta_{0} & =\frac{2}{U}\left(h-h_{0}\right) \\
& =-x .
\end{aligned}
$$

$\Delta_{0}=(-1)^{\mathbf{r}}\left\langle S_{\mathbf{r}}^{x}\right\rangle$ is the AF order parameter in the repulsive model, and the superconducting order parameter in the attractive model. $h$ is an effective magnetic field which takes into account the mean ferromagnetic magnetization $\left\langle c_{\mathbf{r}}^{\dagger} \sigma^{z} c_{\mathbf{r}}\right\rangle$ along the $z$ axis. In the attractive model, $h=h_{0}-x U / 2=\mu+\rho_{0} U / 2$ corresponds to the chemical potential renormalized by the Hartree selfenergy.

Using Eqs. (16), we rewrite the saddle-point equations (1921) as

$$
\begin{aligned}
& \frac{2}{U}=\int_{\mathbf{k}} \frac{\tanh \left(\beta E_{\mathbf{k} \uparrow} / 2\right)}{E_{\mathbf{k} \uparrow}}, \\
& x=\int_{\mathbf{k}} \epsilon_{\mathbf{k} \uparrow} \frac{\tanh \left(\beta E_{\mathbf{k} \uparrow} / 2\right)}{E_{\mathbf{k} \uparrow}},
\end{aligned}
$$
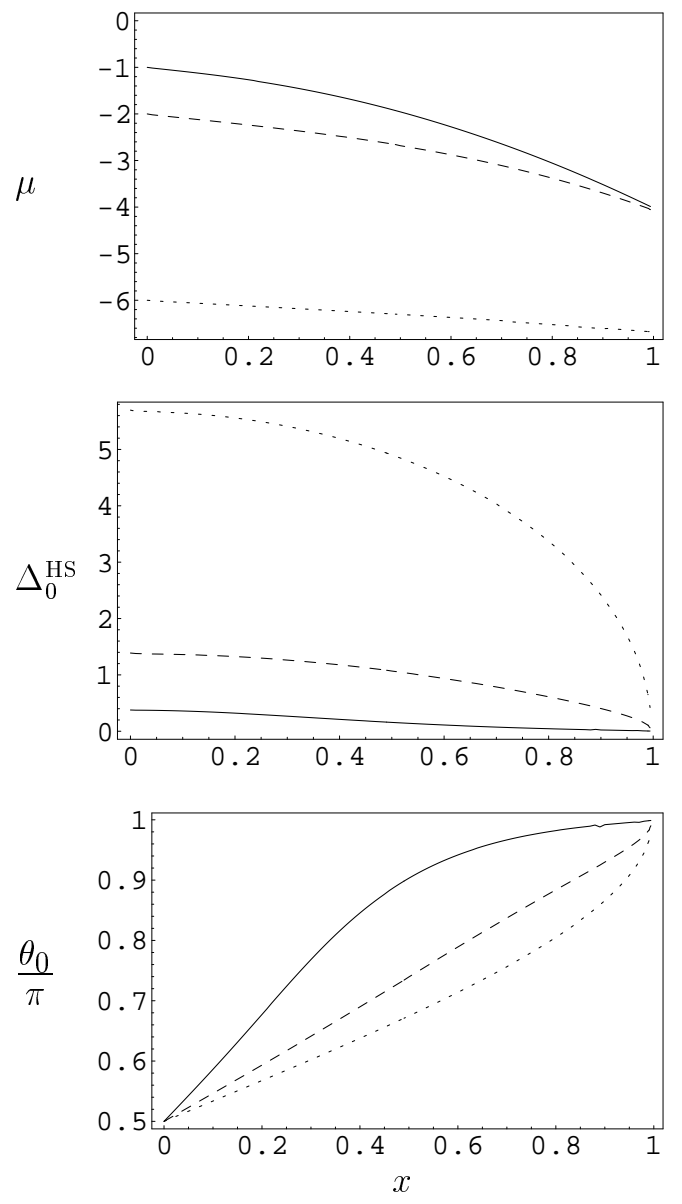

FIG. 2: Chemical potential $\mu=h_{0}-U / 2$, order parameter $\Delta_{0}^{\mathrm{HS}}$ and $\theta_{0}$ in the $T=0$ HF state $v s$ doping $x=1-\rho_{0}$ for $U=$ $2 t, 4 t$ and $12 t$ (solid, dashed, and dotted lines, respectively). The energies are measured in units of $t$.

where $\int_{\mathbf{k}}=\int_{-\pi}^{\pi} \frac{d k_{x}}{2 \pi} \int_{-\pi}^{\pi} \frac{d k_{y}}{2 \pi}$. At $T=0$, Eqs. 2223) determine the superconducting order parameter $\Delta_{0}^{\mathrm{HS}}$ (or, equivalently, $\Delta_{0}$ ) and the renormalized chemical potential $h$. Using (1521), we then obtain $h_{0}, i m_{0}^{\mathrm{HS}}$ and $\theta_{0}$ as a function of $U$ and $x$ [Figs. 223. Eqs. 2223) also determine the $\mathrm{HF}$ transition temperature $T_{c}^{\mathrm{HF}}$ where AF long-range order (i.e. superconducting order in the attractive model) sets in. At the transition, we have $\Delta_{0}=\Delta_{0}^{\mathrm{HS}}=0^{+}, \theta_{0}=\pi$ and $i m_{0}^{\mathrm{HS}}=x U / 2$.

Partial analytical results can be obtained in the limits of weak and strong couplings.

\section{Weak coupling $(U \ll 4 t)$}

At half-filling $(x=0), h=h_{0}=0$ and $\theta_{0}=\pi / 2$. The zero-temperature order parameter and the transition temperature are given by

$$
\begin{aligned}
& \Delta_{0}^{\mathrm{HS}} \simeq 32 t e^{-2 \pi \sqrt{t / U}}, \\
& T_{c}^{\mathrm{HF}} \sim t e^{-2 \pi \sqrt{t / U}} .
\end{aligned}
$$



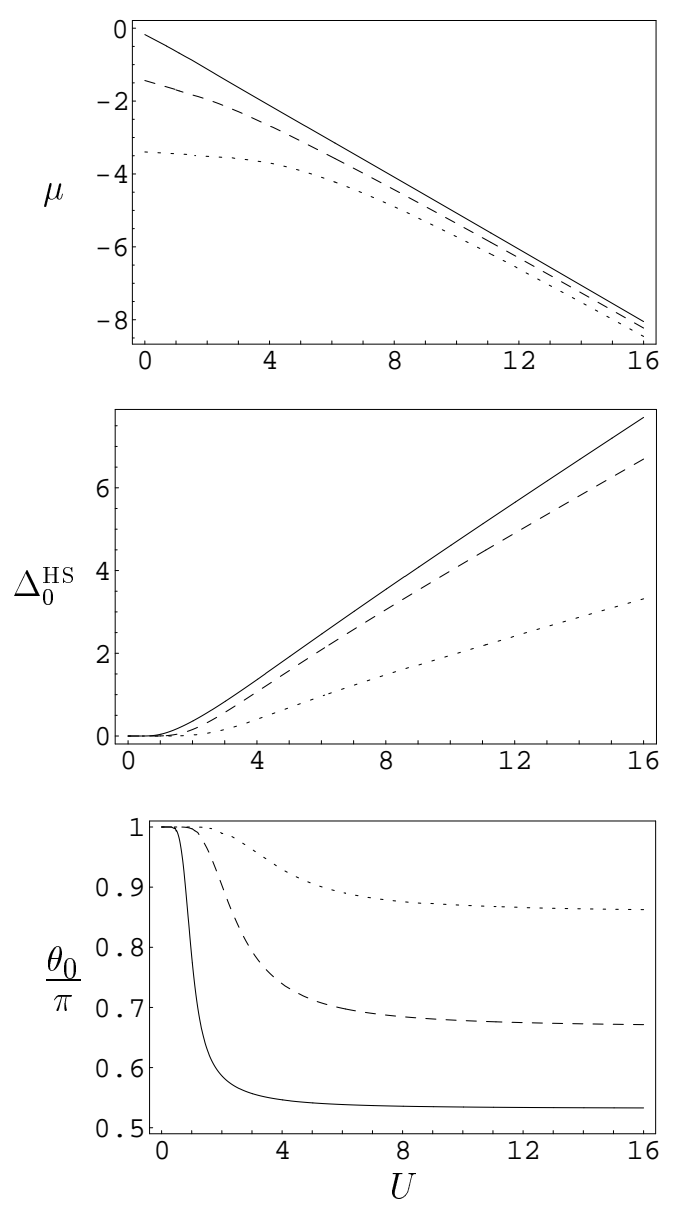

FIG. 3: Chemical potential $\mu=h_{0}-U / 2$, order parameter $\Delta_{0}^{\mathrm{HS}}$ and $\theta_{0}$ in the $T=0 \mathrm{HF}$ state $v s U$ for $x=0.1,0.5$ and 0.9 (solid, dashed, and dotted lines, respectively). Here and in the following figures, we use the analytical results in the weak-coupling regime $(U \lesssim t)$ where the numerics becomes difficult because of the exponentially small value of $\Delta_{0}^{\mathrm{HS}}$.

Note that at half-filling, the $\mathrm{SO}(3)$ symmetry is restored and the AF order parameter can have a component along the $z$ axis. The latter corresponds to a $\mathbf{q}=\mathbf{Q}$ chargedensity-wave order in the attractive model. The choice $\theta_{0}=\pi / 2$ corresponds to a state with only superconducting order.

Away from half-filling $\left(h_{0}<0\right), \theta_{0} \simeq \pi$ and $i m_{0}^{\mathrm{HS}} \simeq$ $x U / 2$ since the superconducting order parameter is exponentially small. At $T=0$, the superconducting order parameter is determined by

$$
\begin{aligned}
\frac{2}{U} & =\int_{-4 t}^{4 t} \frac{d \epsilon}{E} \mathcal{N}_{0}(\epsilon) \\
& \simeq \mathcal{N}_{0}(h) \int_{-4 t}^{4 t} \frac{d \epsilon}{E},
\end{aligned}
$$

where $E=\left[(\epsilon-h)^{2}+\Delta_{0}^{\mathrm{HS}^{2}}\right]^{1 / 2} \cdot \mathcal{N}_{0}(\epsilon)=\left(2 \pi^{2} t\right)^{-1} K[(1-$ $\left.\left.\epsilon^{2} / 16 t^{2}\right)^{1 / 2}\right]$, with $\epsilon \in[-4 t, 4 t]$, is the density of states of free fermions on a square lattice $[K$ is the complete elliptic integral of the first kind]. Since the integral in (25) is peaked around $\epsilon=h$ for $\Delta_{0}^{\mathrm{HS}} \rightarrow 0$, we have replaced the density of states $\mathcal{N}_{0}(\epsilon)$ by its value at the renormalized chemical potential $h$. In the weak-coupling limit, we can also neglect the effect of the order parameter on the chemical potential (i.e. set $\Delta_{0}^{\mathrm{HS}}=0$ in (23)). We then have $h \simeq \epsilon_{F}$ where $\epsilon_{F}$ is the Fermi energy of the non-interacting system. With these approximations, we obtain (for $U \rightarrow 0$ and $x$ fixed)

$$
\begin{aligned}
\Delta_{0}^{\mathrm{HS}} & \simeq 2\left[(4 t)^{2}-\epsilon_{F}^{2}\right]^{1 / 2} e^{-\frac{1}{U \mathcal{N}_{0}\left(\epsilon_{F}\right)}}, \\
x & \simeq \begin{cases}\frac{1}{\pi^{2} t}\left|\epsilon_{F}\right| \ln \frac{16 e t}{\left|\epsilon_{F}\right|} & \text { if } x \ll 1, \\
1-\frac{4 t-\left|\epsilon_{F}\right|}{2 \pi t} & \text { if } 1-x \ll 1,\end{cases} \\
\mu & \simeq \epsilon_{F}-\rho_{0} \frac{U}{2} .
\end{aligned}
$$

Since $h \simeq \epsilon_{F}$ belongs to the non-interacting band $[-4 t, 4 t]$, the excitation gap (i.e. the minimum energy required to break a pair) equals $2 \Delta_{0}^{\mathrm{HS}}$.

Similar arguments show that the transition temperature is given by

$$
T_{c}^{\mathrm{HF}} \simeq \frac{2 \gamma}{\pi}\left[(4 t)^{2}-\epsilon_{F}^{2}\right]^{1 / 2} e^{-\frac{1}{U \mathcal{N}_{0}\left(\epsilon_{F}\right)}},
$$

where $\gamma \simeq 1.78$ is the exponential of the Euler constant. We recover the results of the BCS theory with $\left[(4 t)^{2}-\right.$ $\left.\epsilon_{F}^{2}\right]^{1 / 2}$ playing the role of the cutoff energy [Eqs. (26/27)].

\section{Strong coupling $(U \gg 4 t)$}

To leading order in $1 / U$ (and $x$ fixed), at $T=0$ we find $i m_{0}^{\mathrm{HS}}=U / 2\left(\right.$ or $\left.m_{0}=1\right)$ and $\cos \theta_{0}=-x=h_{0} / 2 J$. This gives

$$
\begin{aligned}
\Delta_{0}^{\mathrm{HS}} & =\frac{U}{2}\left(1-x^{2}\right)^{1 / 2}, \\
\mu & =-\frac{U}{2}-2 J x .
\end{aligned}
$$

In the strong-coupling limit, the excitation gap equals $2\left(h^{2}+\Delta_{0}^{\mathrm{HS}^{2}}\right)^{1 / 2}=U$ to leading order in $1 / U$. For $x \ll 1$, $T_{c}^{\mathrm{HF}} \simeq U / 4$.

\section{B. Effective action $S[m, \boldsymbol{\Omega}]$}

In $2 \mathrm{D}$, the $\mathrm{HF}$ theory breaks down at finite temperature since it predicts $\mathrm{AF}$ long-range order below $T_{c}^{\mathrm{HF}}$. Nevertheless, the HF transition temperature bears a physical meaning as a crossover temperature below which the amplitude $\Delta_{0}$ of the AF order parameter takes a finite value. This can be interpreted as the appearance of local moments perpendicular to the magnetic field and with an amplitude $\Delta_{0}=\Delta_{0}^{\mathrm{HS}} / U$. Note that at weakcoupling these "local" moments can be defined only at length scales of order $\xi_{0} \sim t / \Delta_{0}^{\mathrm{HS}} \gg 1$, which corresponds to the size of bound particle-hole pairs in the HF 
state. Thus, stricto sensu, local moments form only in the strong-coupling limit when $\xi_{0} \sim 1$.

Below $T_{c}^{\mathrm{HF}}$, the fluctuations of the fields $m, m^{\mathrm{HS}}$ and $\theta$ around their $\mathrm{HF}$ values are therefore expected to be small. Below a crossover temperature $T_{X} \leq T_{c}^{\mathrm{HF}}$, AF short-range order sets in. The AF correlation length becomes much larger than the lattice spacing, and the effective action for AF fluctuations (which correspond to rotations of $\Omega_{\mathbf{r}}$ about the $z$ axis) can be derived within a gradient expansion. In this section, we derive the lowenergy effective action $S[m, \boldsymbol{\Omega}]$ for temperatures below the crossover temperature $T_{X}$. For $T \ll T_{X} \leq T_{c}^{\mathrm{HF}}$, the coefficients of the effective action (which are related to HF quantities) can be evaluated in the zero temperature limit. This means that we neglect the exponentially small number of thermally excited quasi-particles which give rise to non-analytic contributions (Landau damping terms) to the effective action 32.33 As shown below, $T_{X} \sim J\left(1-x^{2}\right) / 2 \ll T_{c}^{\mathrm{HF}}$ in the strong-coupling limit $\left(J=4 t^{2} / U\right)$, but $T_{X} \sim T_{c}^{\mathrm{HF}}$ in the weak-coupling limit.

Fluctuations can be parametrized by

$$
\begin{aligned}
\delta m_{\mathbf{r}} & =m_{\mathbf{r}}-m_{0}, \\
\delta m_{\mathbf{r}}^{\mathrm{HS}} & =m_{\mathbf{r}}^{\mathrm{HS}}-m_{0}^{\mathrm{HS}}, \\
p_{\mathbf{r}} & =\frac{\theta_{\mathbf{r}}-\theta_{0}}{2}, \\
q_{\mathbf{r}} & =\varphi_{\mathbf{r}}-\mathbf{Q} \cdot \mathbf{r} .
\end{aligned}
$$

In the HF state, $\delta m_{\mathbf{r}}=\delta m_{\mathbf{r}}^{\mathrm{HS}}=p_{\mathbf{r}}=q_{\mathbf{r}}=0$. The effective action $S\left[p, q, m^{\mathrm{HS}}, m\right]$ is obtained by integrating out the fermions, and assuming $p_{\mathbf{r}}, \delta m_{\mathbf{r}}, \delta m_{\mathbf{r}}^{\mathrm{HS}}$ and $\partial_{\mu} p_{\mathbf{r}}, \partial_{\mu} \delta m_{\mathbf{r}}, \partial_{\mu} \delta m_{\mathbf{r}}^{\mathrm{HS}}, \partial_{\mu} q_{\mathbf{r}}$ to be small $(\mu=0, x, y$ and $\left.\partial_{0} \equiv \partial_{\tau}\right)$. We do not assume $q_{\mathbf{r}}$ to be small so that our approach is valid even in the absence of AF long-range order. It is convenient to introduce a new fermionic variable $\phi_{\mathbf{r}}=\left(\phi_{\mathbf{r} \uparrow}, \phi_{\mathbf{r} \downarrow}\right)^{T}$ defined by $c_{\mathbf{r}}=R_{\mathbf{r}} \phi_{\mathbf{r}}$ where $R_{\mathbf{r}}$ is a time- and site-dependent $\mathrm{SU}(2) / \mathrm{U}(1)$ matrix satisfying

$$
R_{\mathrm{r}} \boldsymbol{\sigma} \cdot \Omega_{\mathrm{r}}^{\mathrm{cl}} R_{\mathrm{r}}^{\dagger}=\boldsymbol{\sigma} \cdot \Omega_{\mathrm{r}}
$$

The above definition means that $\mathcal{R}_{\mathbf{r}}$, the $\mathrm{SO}(3)$ element associated to $R_{\mathbf{r}}$, maps $\Omega_{\mathbf{r}}^{\text {cl }}$ onto $\Omega_{\mathbf{r}}$. The $\mathrm{U}(1)$ gauge freedom is due to rotations around $\boldsymbol{\Omega}_{\mathbf{r}}^{\mathrm{cl}}$, which do not change the physical state of the system. The pseudofermion $\phi_{\mathbf{r}}$ has its spin quantized along $\mathcal{R}_{\mathbf{r}} \hat{\mathbf{z}}$. The action (1112) can then be expressed as

$$
\begin{aligned}
S= & S_{\mathrm{HF}}+S_{1}+S_{2} \\
& +\int_{0}^{\beta} d \tau \sum_{\mathbf{r}}\left(-\frac{U}{4} \delta m_{\mathbf{r}}^{2}+i \delta m_{\mathbf{r}}^{\mathrm{HS}} \delta m_{\mathbf{r}}\right. \\
& \left.-\frac{2}{U} m_{0}^{\mathrm{HS}} \delta m_{\mathbf{r}}^{\mathrm{HS}}\right),
\end{aligned}
$$

where $S_{\mathrm{HF}}$ is the HF action (14) and

$$
\begin{aligned}
& S_{1}=\int_{0}^{\beta} d \tau \sum_{\mathbf{r}} \phi_{\mathbf{r}}^{\dagger} A_{0 \mathbf{r}} \phi_{\mathbf{r}}, \\
& S_{2}=-t \int_{0}^{\beta} d \tau \sum_{\left\langle\mathbf{r}, \mathbf{r}^{\prime}\right\rangle}\left(\phi_{\mathbf{r}}^{\dagger} A_{\mathbf{r}, \mathbf{r}^{\prime}} \phi_{\mathbf{r}^{\prime}}+\text { c.c. }\right) .
\end{aligned}
$$

$\left\langle\mathbf{r}, \mathbf{r}^{\prime}\right\rangle$ denotes nearest neighbors and we have introduced

$$
\begin{aligned}
A_{0 \mathbf{r}} & =R_{\mathbf{r}}^{\dagger} \dot{R}_{\mathbf{r}}-h_{0}\left(R_{\mathbf{r}}^{\dagger} \sigma^{z} R_{\mathbf{r}}-\sigma^{z}\right)-i \delta m_{\mathbf{r}}^{\mathrm{HS}} \boldsymbol{\sigma} \cdot \Omega_{\mathbf{r}}^{\mathrm{cl}} \\
& =\sum_{\nu=x, y, z} A_{0 \mathbf{r}}^{\nu} \sigma^{\nu} \\
A_{\mathbf{r}, \mathbf{r}^{\prime}} & =R_{\mathbf{r}}^{\dagger} R_{\mathbf{r}^{\prime}}-1 \\
& =\sum_{\nu=0, x, y, z} A_{\mathbf{r}, \mathbf{r}^{\prime}}^{\nu} \sigma^{\nu}
\end{aligned}
$$

We use the notation $\dot{R}_{\mathbf{r}}=\partial_{\tau} R_{\mathbf{r}} \cdot \sigma^{0}$ is the $2 \times 2$ unit matrix.

The effective action $S[p, q, m]$ is obtained by integrating out the fermion field $\phi_{\mathbf{r}}$ and the Hubbard Stratonovich field $m_{\mathbf{r}}^{\mathrm{HS}}$. The final result reads

$$
\begin{aligned}
S[p, q, m]= & \frac{1}{2} \sum_{\tilde{q}}\left[p_{-\tilde{q}} \tilde{\Pi}_{p p}(\tilde{q}) p_{\tilde{q}}+q_{-\tilde{q}} \tilde{\Pi}_{q q}(\tilde{q}) q_{\tilde{q}}\right. \\
& +\delta m_{-\tilde{q}} \tilde{\Pi}_{m m}(\tilde{q}) \delta m_{\tilde{q}}+2 p_{-\tilde{q}} \tilde{\Pi}_{p q}(\tilde{q}) q_{\tilde{q}} \\
& \left.+2 p_{-\tilde{q}} \tilde{\Pi}_{p m}(\tilde{q}) \delta m_{\tilde{q}}+2 q_{-\tilde{q}} \tilde{\Pi}_{q m}(\tilde{q}) \delta m_{\tilde{q}}\right] \\
& +\delta S_{B},
\end{aligned}
$$

where $\tilde{q}=\left(\mathbf{q}, i \omega_{\nu}\right)$, with $\omega_{\nu}$ ( $\nu$ integer $)$ a bosonic Matsubara frequency. The coefficients $\tilde{\Pi}$ are given by (B24) and $\delta S_{B}$ by (B21). Details of this rather long calculation are given in Appendix $\mathrm{B}$, to which we refer readers interested in results for the repulsive Hubbard model [Eq. (4)]. In the bulk of the manuscript, we shall focus on the attractive Hubbard model as defined in (11).

\section{Effective action $S[\rho, \Delta]$}

Results of Sec. IIB can be easily translated to the attractive model. Below $T_{\text {pair }} \equiv T_{c}^{\mathrm{HF}}$, the finite amplitude $\Delta_{0}$ of the superconducting order parameter can be interpreted as the appearance of incoherent pairs. At weak coupling, the size $\xi_{0} \sim t / \Delta_{0}^{\mathrm{HS}}$ of these (Cooper) pairs is much larger than the lattice spacing $\left(\xi_{0} \gg 1\right)$. At strong coupling, the preformed pairs are local and are expected to behave as hard-core bosons (the hard-core constraint comes from the Pauli principle which prevents double occupancy of a lattice site). The crossover temperature $T_{X}$ marks the onset of strong superconducting fluctuations (i.e. $\xi \gg 1$ with $\xi$ the superconducting correlation length).

As already pointed out, in general $\frac{m_{\mathbf{r}}}{2} \boldsymbol{\Omega}_{\mathbf{r}}$ cannot be directly identified with the spin density $\mathbf{S}_{\mathbf{r}}=c_{\mathbf{r}}^{\dagger} \frac{\boldsymbol{\sigma}}{2} c_{\mathbf{r}}$. In order to find the relation between $\mathbf{S}_{\mathbf{r}}$ and $\frac{m_{\mathbf{r}}}{2} \boldsymbol{\Omega}_{\mathbf{r}}$, we add to the action the source term

$$
S_{J}=\int_{0}^{\beta} d \tau \sum_{\mathbf{r}} c_{\mathbf{r}}^{\dagger} \mathbf{J}_{\mathbf{r}} \cdot \boldsymbol{\sigma} c_{\mathbf{r}}
$$

with $\mathbf{J}_{\mathbf{r}}=\left(J_{\mathbf{r}}^{x}, J_{\mathbf{r}}^{y}, J_{\mathbf{r}}^{z}\right)$. The charge and pairing fields in 
the attractive model are then obtained from [see Eq. (7)]

$$
\begin{aligned}
&\left.\frac{\delta S}{\delta J_{\mathbf{r}}^{x}}\right|_{J=0} \equiv 2(-1)^{\mathbf{r}}\left|\Delta_{\mathbf{r}}\right| \cos \Theta_{\mathbf{r}} \\
&\left.\frac{\delta S}{\delta J_{\mathbf{r}}^{y}}\right|_{J=0} \equiv-2(-1)^{\mathbf{r}}\left|\Delta_{\mathbf{r}}\right| \sin \Theta_{\mathbf{r}} \\
&\left.\frac{\delta S}{\delta J_{\mathbf{r}}^{z}}\right|_{J=0} \equiv \rho_{\mathbf{r}}-1
\end{aligned}
$$

We then proceed as in the preceding section (see also Appendix (B). We integrate out the fermions to obtain the effective action to second order in $p_{\mathbf{r}}, \delta m_{\mathbf{r}}, \partial_{\mu} p_{\mathbf{r}}, \partial_{\mu} \delta m_{\mathbf{r}}, \partial_{\mu} q_{\mathbf{r}}$ and first order in $\mathbf{J}_{\mathbf{r}}$. Using then Eqs. (36), we obtain the relation between $m_{\mathbf{r}}, \boldsymbol{\Omega}_{\mathbf{r}}$ and $\Delta_{\mathbf{r}}, \rho_{\mathbf{r}}$ : see Eq. (D6) in Appendix D. Eq. (D6) takes a simple form in the BCS and Bose limits (see Secs. IIC1 and $\amalg \mathrm{C2}_{2}$.

To proceed further in the general case, we note that there is no coupling between $\boldsymbol{\nabla} q$ and $p, \dot{q}, m$ in the action $S[p, q, m]$ (Appendix B). Since $\nabla \Theta_{\mathbf{r}}=-\nabla q_{\mathbf{r}}$, and $\dot{\Theta}, \delta \rho, \delta|\Delta|$ are functions of $p, \dot{q}, m$ (Appendix (D), $S[\rho, \Delta]$ takes the general form

$$
\begin{aligned}
S[\rho, \Delta]= & \frac{1}{2} \int_{0}^{\beta} d \tau \int d^{2} r\left[i \rho_{\mathbf{r}} \dot{\Theta}_{\mathbf{r}}+\frac{\langle-K\rangle}{8}\left(\nabla \Theta_{\mathbf{r}}\right)^{2}\right. \\
& +\Pi_{\rho \rho}\left(\delta \rho_{\mathbf{r}}\right)^{2}+\Pi_{|\Delta||\Delta|}\left(\delta\left|\Delta_{\mathbf{r}}\right|\right)^{2} \\
& \left.+2 \Pi_{\rho|\Delta|} \delta \rho_{\mathbf{r}} \delta\left|\Delta_{\mathbf{r}}\right|\right]
\end{aligned}
$$

Here we use the fact that half the fermion density is the conjugate variable of the phase of the superconducting order parameter, as required by gauge invariance ${ }^{34}$ The action $S[\rho, \Delta]$ was previously obtained in Ref. 12. For our purpose, it is not necessary to determine the expression of the coefficients $\Pi_{\rho \rho}, \Pi_{|\Delta||\Delta|}$ and $\Pi_{\rho|\Delta|}$ which can be found in Ref. 12. ${ }^{35}$ To make contact with the usual description of a Bose superfluid, we integrate out the amplitude field $|\Delta|$. We thus obtain the action

$$
\begin{aligned}
S[\rho, \Theta]= & \frac{1}{2} \int_{0}^{\beta} d \tau \int d^{2} r\left\{i \rho_{\mathbf{r}} \dot{\Theta}_{\mathbf{r}}+\frac{\langle-K\rangle}{8}\left(\nabla \Theta_{\mathbf{r}}\right)^{2}\right. \\
& \left.+\left(\delta \rho_{\mathbf{r}}\right)^{2}\left[\Pi_{\rho \rho}-\frac{\Pi_{\rho|\Delta|}^{2}}{\Pi_{|\Delta||\Delta|}}\right]\right\} .
\end{aligned}
$$

This action is similar to the superfluid action ${ }^{36}$

$$
S_{b}=\int_{0}^{\beta} d \tau \int d^{2} r\left[i \rho_{b \mathbf{r}} \dot{\Theta}_{\mathbf{r}}+\frac{\rho_{b 0}}{2 m_{b}}\left(\nabla \Theta_{\mathbf{r}}\right)^{2}+\frac{g}{2}\left(\delta \rho_{b \mathbf{r}}\right)^{2}\right]
$$

for bosons of mass $m_{b}$ and density $\rho_{b \mathbf{r}}$ ( $\rho_{b 0}$ is the mean density and $\left.\delta \rho_{b \mathbf{r}}=\rho_{b \mathbf{r}}-\rho_{b 0}\right) . g$ is the amplitude of the repulsive interaction between bosons. Comparing Eqs. (38) and (39), we obtain

$$
\begin{aligned}
\rho_{b \mathbf{r}} & =\frac{\rho_{\mathbf{r}}}{2}, \\
m_{b} & =\frac{4 \rho_{0}}{\langle-K\rangle}, \\
g & =4\left(\Pi_{\rho \rho}-\frac{\Pi_{\rho|\Delta|}^{2}}{\Pi_{|\Delta||\Delta|}}\right) .
\end{aligned}
$$

In order to calculate $g$ without computing $\Pi_{\rho \rho}, \Pi_{|\Delta||\Delta|}$ and $\Pi_{\rho|\Delta|}$, we integrate out charge fluctuations. This yields the phase-only action

$$
\begin{aligned}
S[\Theta]= & \int_{0}^{\beta} d \tau \int d^{2} r\left[\frac{\rho_{0}}{4 m_{b}}\left(\nabla \Theta_{\mathbf{r}}\right)^{2}+\frac{\dot{\Theta}_{\mathbf{r}}^{2}}{2 g}\right] \\
& +\frac{i}{2} \rho_{0} \int_{0}^{\beta} d \tau \int d^{2} r \dot{\Theta}_{\mathbf{r}} .
\end{aligned}
$$

$S[\Theta]$ corresponds to an $\mathrm{O}(2)$ sigma model with an additional term proportional to the first-order time derivative of $\Theta . m_{b}$ and $g$ are related to the phase stiffness $\rho_{s}^{0}$ and the velocity $c$ of the $\mathrm{O}(2)$ sigma model:

$$
\begin{aligned}
m_{b} & =\frac{\rho_{0}}{2 \rho_{s}^{0}}, \\
g & =\frac{c^{2}}{\rho_{s}^{0}} .
\end{aligned}
$$

$S[\Theta]$ can be directly obtained from the action $S[p, q, m]$ (Sec.IID), which allows to determine $\rho_{s}^{0}$ and $c$, and therefore $m_{b}$ and $g$. Anticipating on the results of Sec. IID we show in Figs. 45 $m_{b}$ and $g$ as a function of doping and interaction strength. Because both $\rho_{s}^{0}$ and $c$ vanish in the low-density limit, the numerical determination of $m_{b}$ and $g$ from (42) is difficult when $x \rightarrow 1$. We have therefore only considered $x \leq 0.9$.

The low-energy effective action (38 39) ensures that the Fermi superfluid will behave similarly to a Bose superfluid. Indeed, from the classical equation of motion, we obtain the two basic equations (in imaginary time) of a superfluid ${ }^{36}$

$$
\begin{aligned}
i \dot{\rho}_{b \mathbf{r}}+\nabla \cdot( & \left.\frac{\rho_{b 0}}{m_{b}} \nabla \Theta_{\mathbf{r}}\right)=0 \\
i \dot{\Theta}_{\mathbf{r}}+g \delta \rho_{b \mathbf{r}} & =0 .
\end{aligned}
$$

For a Bose superfluid, the coefficient in front of $(\nabla \Theta)^{2}$ and $\nabla \Theta$ in Eqs. (39) and (43) is the superfluid density (divided by the boson mass). Here, because the coefficients of the effective action $S[\rho, \Theta]$ are calculated from the HF action, we expect the superfluid density to be given by the full density $\rho_{b 0}=\rho_{0} / 2$. In Sec.IV we derive the effective action $S[\rho, \Theta]$ in the strong-coupling lowdensity limit [including the terms proportional to $\left(\boldsymbol{\nabla} \rho_{\mathbf{r}}\right)^{2}$ that are omitted in (39)], without relying on the HF theory. We obtain a boson mass $m_{b}=1 / J$ in agreement with (40) when $U \gg 4 t$ (see Eq. (50) below for $x \simeq 1$ ). 

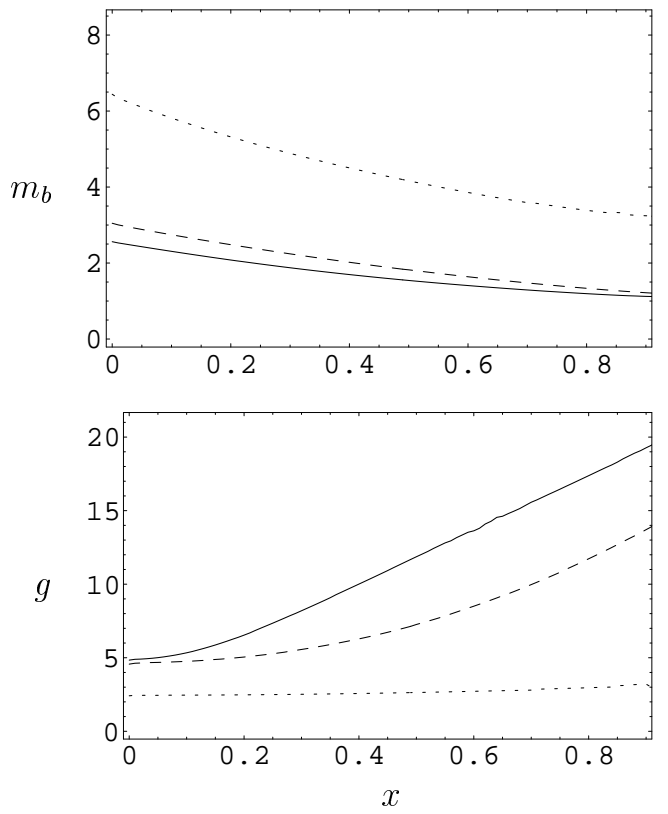

FIG. 4: Boson mass $m_{b}$ and interaction strength $g$ vs doping $x=1-\rho_{0}$ for $U=2 t, 4 t$ and $12 t$ (solid, dashed, and dotted lines, respectively).
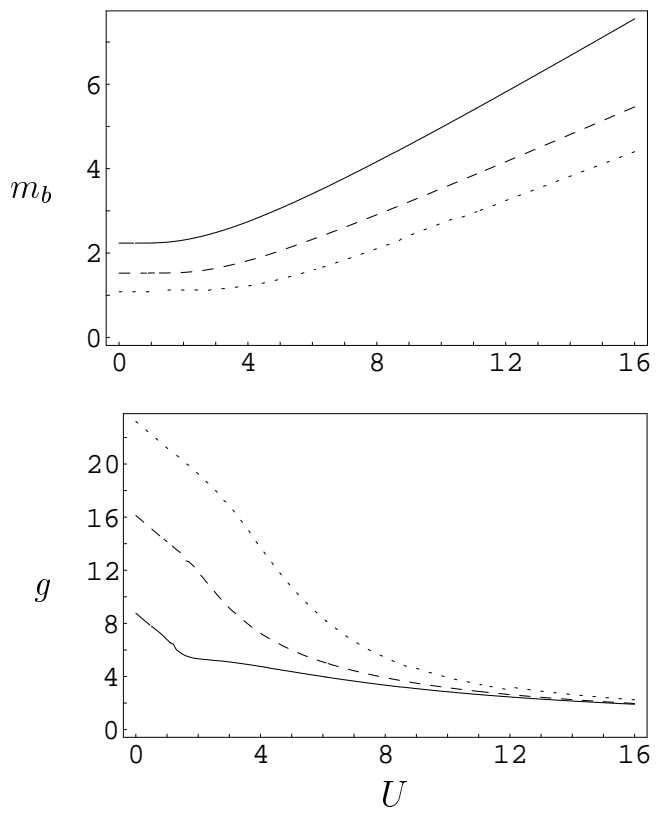

FIG. 5: Boson mass $m_{b}$ and interaction strength $g$ vs $U$ for $x=0.1,0.5$ and 0.9 (solid, dashed, and dotted lines, respectively).

There is nevertheless an important difference with the Bose superfluid case. $S[\rho, \Theta]$ has been obtained by integrating out amplitude fluctuations $(|\Delta|)$ and therefore does not describe the full dynamics of the superconducting order parameter $\Delta_{\mathbf{r}}=\left|\Delta_{\mathbf{r}}\right| e^{i \Theta_{\mathbf{r}}}$ (except in the strong coupling limit, see Secs. II 2 2 and IV.

Effective actions similar to (38) have been derived previously for continuum or lattice models $\frac{1.10 .11 .12}{2}$ In this section, we have obtained an effective action for the lattice case which is valid for all values of the interaction strength. The validity of our approach is quite obvious both at weak and strong couplings. In the latter limit, it simply follows from the mapping between the attractive model and the Heisenberg model under the particle-hole transformation (3) (see Sec.IV]). We therefore expect our approach to provide a good description of the BCS-Bose crossover as the interaction strength increases.

We discuss below in more detail the BCS and Bose limits.

\section{BCS limit} (D6)

Using the results of Appendix B 1 we deduce ${ }^{37}$ from

$$
\begin{aligned}
\delta \rho_{\mathbf{r}} & =-\delta m_{\mathbf{r}}, \\
\delta\left|\Delta_{\mathbf{r}}\right| & =-p_{\mathbf{r}} x .
\end{aligned}
$$

Eqs. (44) can be directly obtained by assuming $\mathbf{S}_{\mathbf{r}}=$ $\frac{m_{\mathrm{r}}}{2} \boldsymbol{\Omega}_{\mathrm{r}}$ and considering the limit $\Delta_{0} \rightarrow 0$. This shows that the identification between $\mathbf{S}_{\mathbf{r}}$ and $\frac{m_{\mathbf{r}}}{2} \Omega_{\mathbf{r}}$ holds in the weak-coupling limit. When inverting the particle-hole transformation (3), the kinetic energy remains unchanged and the HF spin susceptibility $\Pi_{00}^{z z}(0,0)$ becomes the HF compressibility $\kappa$ of the attractive model. The effective action $S[q, m]$ [Eq. (B28)] then becomes

$$
\begin{aligned}
S[\rho, \Theta]= & \frac{1}{2} \int_{0}^{\beta} d \tau \int d^{2} r\left[i \rho_{\mathbf{r}} \dot{\Theta}_{\mathbf{r}}+\frac{\langle-K\rangle}{8}\left(\nabla \Theta_{\mathbf{r}}\right)^{2}\right. \\
& \left.+\left(\frac{1}{\kappa}-\frac{U}{2}\right)\left(\delta \rho_{\mathbf{r}}\right)^{2}\right] .
\end{aligned}
$$

Amplitude fluctuations $(|\Delta|)$ do not couple to density and phase fluctuations in the weak-coupling limit. We also verify that half the fermion density is the conjugate variable of the phase $\Theta$ of the pairing field. The gauge choice $\psi_{\mathbf{r}}=\varphi_{\mathbf{r}} / m_{0}$ [Eq. [B21] ] is crucial to obtain this result. For instance, with $\psi_{\mathbf{r}}=0$, one would obtain $\left(\rho_{\mathbf{r}}-1\right) / 2$ as the conjugate variable of $\Theta_{\mathbf{r}}$.

From (45), we conclude that in the BCS limit the system behaves as a Bose superfluid with $\rho_{b \mathbf{r}}=\rho_{\mathbf{r}} / 2$ and

$$
\begin{aligned}
m_{b} & =4 \frac{\rho_{0}}{\langle-K\rangle}, \\
g & =4\left(\frac{1}{\kappa}-\frac{U}{2}\right) .
\end{aligned}
$$

\section{Bose limit}

In the strong-coupling limit, the auxiliary field $\frac{m_{r}}{2} \boldsymbol{\Omega}_{\mathbf{r}}$ can be identified with the spin density $\mathbf{S}_{\mathbf{r}}$ (see Appendix D1) so that

$$
\begin{aligned}
\rho_{\mathbf{r}}-1 & =\Omega_{\mathbf{r}}^{z}, \\
\Delta_{\mathbf{r}} & =\frac{(-1)^{\mathbf{r}}}{2} \Omega_{\mathbf{r}}^{-},
\end{aligned}
$$


where $\Omega_{\mathbf{r}}^{ \pm}=\Omega_{\mathbf{r}}^{x} \pm i \Omega_{\mathbf{r}}^{y}$. The condition $\Omega_{\mathbf{r}}^{2}=1$ implies that charge and amplitude fluctuations are not independent but tied by the relation

$$
\left|\Delta_{\mathbf{r}}\right|=\frac{1}{2} \sqrt{\rho_{\mathbf{r}}\left(2-\rho_{\mathbf{r}}\right)}
$$

There is therefore a one-to-one correspondence between the bosonic field $\Psi_{\mathbf{r}}=\sqrt{\rho_{\mathbf{r}} / 2} e^{i \Theta_{\mathbf{r}}}$ and the pairing field $\Delta_{\mathbf{r}}=\left|\Delta_{\mathbf{r}}\right| e^{i \Theta_{\mathbf{r}}}$. In the low-density limit, both fields coincide, since $\left|\Delta_{\mathbf{r}}\right| \simeq \sqrt{\rho_{\mathbf{r}} / 2}$.

From (47), we deduce $\delta \rho_{\mathbf{r}}=-2 \sin \theta_{0} p_{\mathbf{r}}$ and $\delta\left|\Delta_{\mathbf{r}}\right|=$ $\cos \theta_{0} p_{\mathbf{r}}$ (with $\cos \theta_{0}=-x$ ). These equations can also be obtained from (D6). The effective action $S[\rho, \Theta]$ then reads [see Eq. (B333)]

$$
\begin{aligned}
S[\rho, \Theta]= & \frac{1}{2} \int_{0}^{\beta} d \tau \int d^{2} r\left[i \rho_{\mathbf{r}} \dot{\Theta}_{\mathbf{r}}+\frac{J}{4}\left(1-x^{2}\right)\left(\nabla \Theta_{\mathbf{r}}\right)^{2}\right. \\
& \left.+2 J\left(\delta \rho_{\mathbf{r}}\right)^{2}\right] .
\end{aligned}
$$

Again we verify that half the fermion density is the conjugate variable of the phase $\Theta$ of the pairing field. In the Bose limit, we therefore have

$$
\begin{aligned}
m_{b} & =\frac{2}{J(1+x)}, \\
g & =8 J .
\end{aligned}
$$

The BCS and Bose limits differ in the role of the Berry phase term

$$
\left\langle\phi_{\mathbf{r}}^{\dagger} R_{\mathbf{r}}^{\dagger} \dot{R}_{\mathbf{r}} \phi_{\mathbf{r}}\right\rangle=i \Delta_{0}\left(p_{\mathbf{r}} \dot{q}_{\mathbf{r}}-\dot{p}_{\mathbf{r}} q_{\mathbf{r}}\right)-\frac{i}{2} \rho_{0} \dot{q}_{\mathbf{r}},
$$

where we have included the gauge-dependent contribution (B20). For $U \gg 4 t$, the Berry phase term becomes $\frac{i}{2} \rho_{\mathbf{r}} \dot{\Theta}_{\mathbf{r}}$ and therefore determines the entire dynamics of the phase $\Theta$. This is expected since the dynamics of the Heisenberg model entirely comes from the Berry phase term ${ }^{38}$ For $U \ll 4 t$, the Berry phase term only gives $\frac{i}{2} \rho_{0} \dot{\Theta}_{\mathbf{r}}$ and does not contribute to the $T=0$ phase collective mode. The missing term, $\frac{i}{2} \delta \rho_{\mathbf{r}} \dot{\Theta}_{\mathbf{r}}$, comes from the second-order cumulant $\left\langle\left(S_{1}+S_{2}\right)^{2}\right\rangle_{c}$. For all values of the interaction strength, the contribution $\frac{i}{2} \rho_{0} \int_{0}^{\beta} d \tau \sum_{\mathbf{r}} \dot{\Theta}_{\mathbf{r}}$ to the action, which is responsible for the Magnus force acting a vortex ${ }^{10}$ is given by the Berry phase term of the effective action $S[m, \Omega]$, 39

\section{Phase-only action $S[\Theta]$}

Integrating out $p, \delta m^{\mathrm{HS}}$ and $\delta m$ in the action $S\left[p, q, m^{\mathrm{HS}}, m\right]$, and using $\Theta_{\mathbf{r}}=-q_{\mathbf{r}}$, we obtain the phase-only action

$$
\begin{aligned}
S[\Theta]= & \frac{\rho_{s}^{0}}{2} \int_{0}^{\beta} d \tau \int d^{2} r\left[\left(\nabla \Theta_{\mathbf{r}}\right)^{2}+\frac{\dot{\Theta}_{\mathbf{r}}^{2}}{c^{2}}\right] \\
& +\frac{i}{2} \rho_{0} \int_{0}^{\beta} d \tau \int d^{2} r \dot{\Theta}_{\mathbf{r}},
\end{aligned}
$$
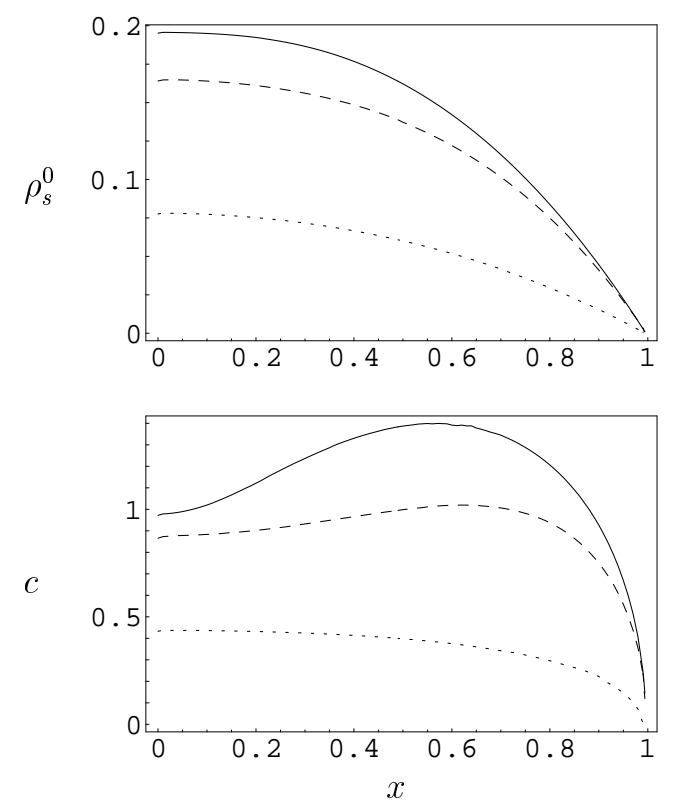

FIG. 6: Phase stiffness $\rho_{s}^{0}$ and velocity $c$ vs doping $x=1-$ $\rho_{0}$ for $U=2 t, 4 t$ and $12 t$ (solid, dashed, and dotted lines, respectively).

where we have taken the continuum limit in real space. Eq. (52) is valid in the hydrodynamic regime defined by the momentum-space cutoff $\Lambda \sim \min \left(1,2 \Delta_{0}^{\mathrm{HS}} / c\right)$. Its validity also requires the fluctuations of $\theta$ to be small, a condition which is not fulfilled in the vicinity of halffilling. This case is discussed in detail in Sec. IIII The (bare) phase stiffness $\rho_{s}^{0}$ and the velocity $c$ of the $\mathrm{O}(2)$ sigma model in (52) are determined by

$$
\begin{aligned}
& \rho_{s}^{0}=\frac{\langle-K\rangle}{8}, \\
& \frac{\rho_{s}^{0}}{c^{2}}=\frac{\Pi_{00}^{z z}(0,0)}{4}+\lim _{\tilde{q} \rightarrow 0} \frac{1}{\omega_{\nu}^{2}}\left[\frac{\Pi_{q m^{\mathrm{HS}}}^{2}(\tilde{q})}{\Pi_{m^{\mathrm{HS}} m^{\mathrm{HS}}}^{\prime}(\tilde{q})}\right. \\
& \left.+\frac{\left(\Pi_{q p}(\tilde{q})-\frac{\Pi_{q m \mathrm{HS}}(\tilde{q}) \Pi_{m} \mathrm{HS}_{p}(\tilde{q})}{\Pi_{m}^{\prime} \mathrm{HS}_{m} \mathrm{HS}(\tilde{q})}\right)^{2}}{\Pi_{p p}(\tilde{q})-\frac{\Pi_{p m}^{2} \mathrm{HS}(\tilde{q})}{\Pi_{m}^{\prime} \mathrm{HS}_{m} \mathrm{HS}(\tilde{q})}}\right] .
\end{aligned}
$$

At zero temperature, there is superconducting long-range order (in the attractive model). From (52), we deduce the existence of a gapless (Goldstone) mode with dispersion $\omega=c|\mathbf{q}|$. This collective mode smoothly evolves from the Anderson-Bogoliubov mode $\mathrm{e}^{30}$ at weak coupling to the Bogoliubov mode ${ }^{31}$ of a Bose superfluid at strong coupling (see Secs. IID 1 IID 2).

Figs. 6.7 show $c$ and $\rho_{s}^{0}$ vs $U$ and $x$. Our results reproduce the collective mode velocity obtained from a random-phase-approximation (RPA) calculation about the zero-temperature HF superconducting state. $\underline{40.41 .42}$ 

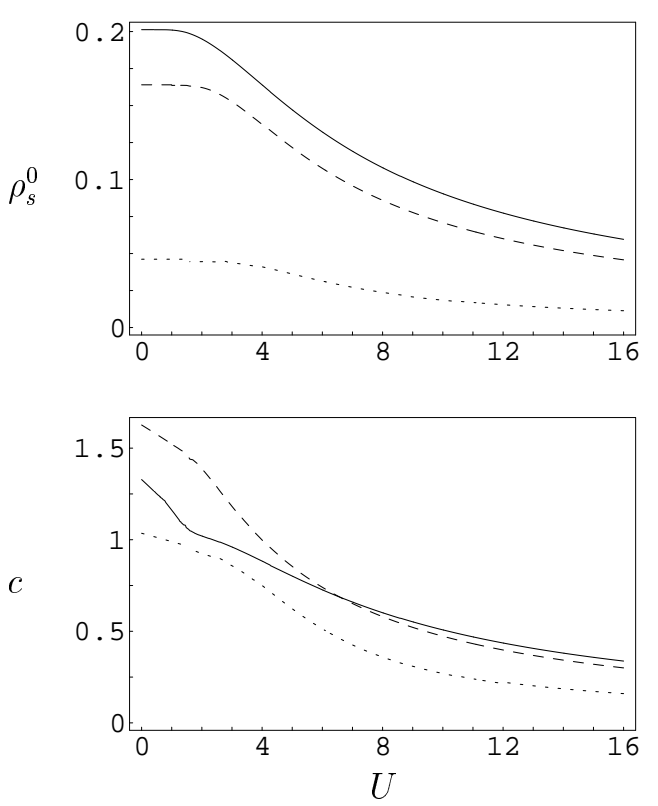

FIG. 7: Phase stiffness $\rho_{s}^{0}$ and velocity $c$ vs $U$ for $x=0.1,0.5$ and 0.9 (solid, dashed, and dotted lines, respectively).

\section{BCS limit}

In the weak-coupling limit, using the results of Appendix B1 and Sec. IIC 1 we obtain

$$
c^{2}=\frac{4 \rho_{s}^{0}}{\kappa}\left(1-\frac{U}{2} \kappa\right) .
$$

We further simplify this result by noting that $\Delta_{0}^{\mathrm{HS}^{2}} /[(\epsilon-$ $\left.\left.\epsilon_{F}\right)^{2}+\Delta_{0}^{\mathrm{HS}^{2}}\right]^{3 / 2} \equiv 2 \delta\left(\epsilon-\epsilon_{F}\right)$ in the limit $\Delta_{0}^{\mathrm{HS}} \rightarrow 0$. This yields [see Eqs. A7] in Appendix A

$$
\kappa=2 \mathcal{N}_{0}\left(\epsilon_{F}\right) \text {. }
$$

Using also (Appendix C)

$$
\langle-K\rangle=2 \int_{\mathbf{k}} v_{\mathbf{k}}^{2} \delta\left(\epsilon_{\mathbf{k}}-\epsilon_{F}\right),
$$

where $\mathbf{v}_{\mathbf{k}}=\nabla_{\mathbf{k}} \epsilon_{\mathbf{k}}$, we finally obtain

$$
\begin{aligned}
c^{2} & =\frac{\overline{v_{\mathbf{k}}^{2}}}{2}\left[1-U \mathcal{N}_{0}\left(\epsilon_{F}\right)\right], \\
\overline{v_{\mathbf{k}}^{2}} & =\frac{\int_{\mathbf{k}} v_{\mathbf{k}}^{2} \delta\left(\epsilon_{\mathbf{k}}-\epsilon_{F}\right)}{\int_{\mathbf{k}} \delta\left(\epsilon_{\mathbf{k}}-\epsilon_{F}\right)} .
\end{aligned}
$$

$\overline{v_{\mathbf{k}}^{2}}$ is the mean square velocity on the (non-interacting) Fermi surface. Eqs. (57) reproduce the expression of the velocity of the Anderson-Bogoliubov mode ${ }^{30}$ in a $2 \mathrm{D}$ continuum model if we identify $\overline{v_{\mathbf{k}}^{2}}$ with $v_{F}^{2}=\left(k_{F} / m\right)^{2}$ (with $k_{F}$ the Fermi momentum and $m$ the fermion mass). They were also obtained in Ref. 41 from an RPA calculation about the zero-temperature HF superconducting state.

\section{Bose limit}

In the strong-coupling limit, using the results of Appendix B2 we obtain

$$
\begin{aligned}
\rho_{s}^{0} & =\frac{J}{4}\left(1-x^{2}\right), \\
c & =\sqrt{2} J \sqrt{1-x^{2}} .
\end{aligned}
$$

We recover the velocity $c=\sqrt{\rho_{b 0} g / m_{b}}$ of the Bogoliubov mode in a Bose superfluid, ${ }^{36}$ where the mass $m_{b}$ and the interaction amplitude $g$ of the bosons are defined in (50) $\left(\rho_{b 0}=\rho_{0} / 2\right)$. At half-filling, $x=0$, Eqs. (58) agree with results obtained directly from the Heisenberg model.

\section{E. Berezinskii-Kosterlitz-Thouless transition}

The effective action $S[\Theta]$ derived in Sec.IID is valid for temperatures below the crossover temperature $T_{X}$, i.e. $T \ll T_{X}$ (see Sec. IIB). Since $T_{\mathrm{BKT}} \sim T_{X} \sim T_{\text {pair }}$ in the weak-coupling limit (as will be shown below), this effective action is not sufficient if one is interested in the BKT phase transition. In this section, we derive the phase-only action $S[\Theta]$ for temperatures $T \leq T_{\text {pair }}$ and obtain the BKT phase transition temperature $T_{\mathrm{BKT}}$. We consider the static (i.e. classical) limit, where non-analytic contributions due to the Landau damping terms ${ }^{32.33}$ are not present. In this limit, the $q$ field decouple from other fluctuations $\left(p, \delta m^{\mathrm{HS}}\right.$ and $\left.\delta m\right)$, and we obtain (see Appendix A.

$$
\begin{aligned}
S[\Theta] & =\frac{\rho_{s}^{0}}{2 T} \int d^{2} r\left(\nabla \Theta_{\mathbf{r}}\right)^{2} \\
\rho_{s}^{0} & =\frac{\langle-K\rangle}{8}-\frac{\Pi_{x x}^{z z}(0,0)}{4} \\
& =\int_{\mathbf{k}}\left[\frac{\epsilon_{\mathbf{k}} \epsilon_{\mathbf{k} \uparrow}}{8 E_{\mathbf{k} \uparrow}} \tanh \frac{E_{\mathbf{k} \uparrow}}{2 T}-\frac{t^{2} \sin ^{2} k_{x}}{2 T \cosh ^{2}\left(\frac{E_{\mathbf{k} \uparrow}}{2 T}\right)}\right]
\end{aligned}
$$

The action (59) is valid in the hydrodynamic regime and must therefore be supplemented with a momentum-space cutoff $\Lambda \sim \min \left(1,2 \Delta_{0}^{\mathrm{HS}}(T) / c\right)$. $\Pi_{x x}^{z z}(0,0)$ is a currentcurrent correlation function in the attractive model. It vanishes at zero temperature, and the phase stiffness reduces to the mean kinetic energy as obtained in Sec. IID. The expression (60) of the finite-temperature phase stiffness has also been obtained in Refs. 43.44.45.

In order to extract the BKT phase transition temperature, we assume that the system is described by an XY model whose continuum limit is given by (59) 43.44 .45 .46 .47 Although this assumption can be justified at strongcoupling (except in the low-density limit) (see Sec. IV], it is in general not correct. ${ }^{18}$ Nevertheless, it should give a reasonable estimate of the BKT phase transition temperature. This leads us to the 2D XY Hamiltonian

$$
H_{X Y}=-\rho_{s}^{0} \sum_{\left\langle\mathbf{r}, \mathbf{r}^{\prime}\right\rangle} \cos \left(\Theta_{\mathbf{r}}-\Theta_{\mathbf{r}^{\prime}}\right)
$$


defined on a square lattice with spacing $\sim \Lambda^{-1}$. The XY model (61) is known to have a BKT phase transition at the temperature $T_{\mathrm{BKT}}$ defined by

$$
T_{\mathrm{BKT}}=Q \rho_{s}^{0}\left(T_{\mathrm{BKT}}\right)
$$

where $Q \simeq 0.898$ is estimated from Monte-Carlo simulations. ${ }^{48.49 .50}$ In (62), we have written explicitly the temperature dependence of $\rho_{s}^{0}$. $T_{\mathrm{BKT}}$ is obtained by solving simultaneously Eqs. (22/2362).

From the XY model, we can extract another characteristic temperature,

$$
T_{X}=2 \rho_{s}^{0}\left(T_{X}\right)
$$

defined as the mean-field transition temperature of $H_{X Y}$. $T_{X}$ marks the onset of a $\mathrm{RC}$ regime of superconducting fluctuations. In this regime, superconducting fluctuations become quasi-static, the amplitude $\left|\Delta_{\mathbf{r}}\right|$ of the order parameter takes a finite value, and strong phase fluctuations develop (i.e. $\xi \gg 1$, with $\xi$ the correlation length) 51

In the strong coupling limit, $T_{\mathrm{BKT}} \simeq Q \frac{J}{4}\left(1-x^{2}\right)$ and $T_{X} \simeq \frac{J}{2}\left(1-x^{2}\right) \ll T_{\text {pair. }}$. In the weak-coupling limit, $T_{\mathrm{BKT}} \sim T_{X} \sim T_{\text {pair }} \propto e^{-1 / U \mathcal{N}_{0}\left(\epsilon_{F}\right)}$ is exponentially small.

\section{F. Phase diagram}

Near half-filling, $\mathbf{q}=\mathbf{Q}$ charge fluctuations become important and are suppressed only at very small temperature. [This corresponds to a weak magnetic field $h_{0}$ in the repulsive model.] We call $T_{\mathrm{SO}(3 \rightarrow 2)}$ the crossover temperature above which charge fluctuations restore the $\mathrm{SO}(3)$ symmetry of the order parameter. Postponing the determination of $T_{\mathrm{SO}(3 \rightarrow 2)}$ to Sec. III we discuss in this section the phase diagram of the $2 \mathrm{D}$ attractive Hubbard model.

Let us first discuss the phase diagram as a function of doping (Figs. 89). Below $T_{\text {pair }}$ (not shown in the figure for $U=12 t$ ), the amplitude of the superconducting order parameter takes a finite value, but with no short-range order of the phase (i.e. $\xi \sim 1$ with $\xi$ the superconducting correlation length). This corresponds to the appearance of incoherent pairs (Cooper pairs at weak-coupling and local pairs at strong coupling). Below $T_{X}$, superconducting correlations start to grow. The system enters a $\mathrm{RC}$ regime of fluctuations with $\mathrm{SO}(2)$ symmetry: the phase correlation length $\xi$ increases and rapidly becomes much larger than the lattice spacing $(\xi \gg 1)$. At $T_{\mathrm{BKT}}$, a BKT phase transition occurs and the system becomes superconducting. Superconducting long-range order sets in at $T=0$. The situation is slightly different near half-filling where $T_{\mathrm{SO}(3 \rightarrow 2)}<T_{X}$. Below $T_{X}$, both superconducting and $\mathbf{q}=\mathbf{Q}$ charge fluctuations start to grow, and the order parameter has an effective $\mathrm{SO}(3)$ symmetry. For $T<T_{\mathrm{SO}(3 \rightarrow 2)}$, charge fluctuations are suppressed and only superconducting fluctuations continue to grow.
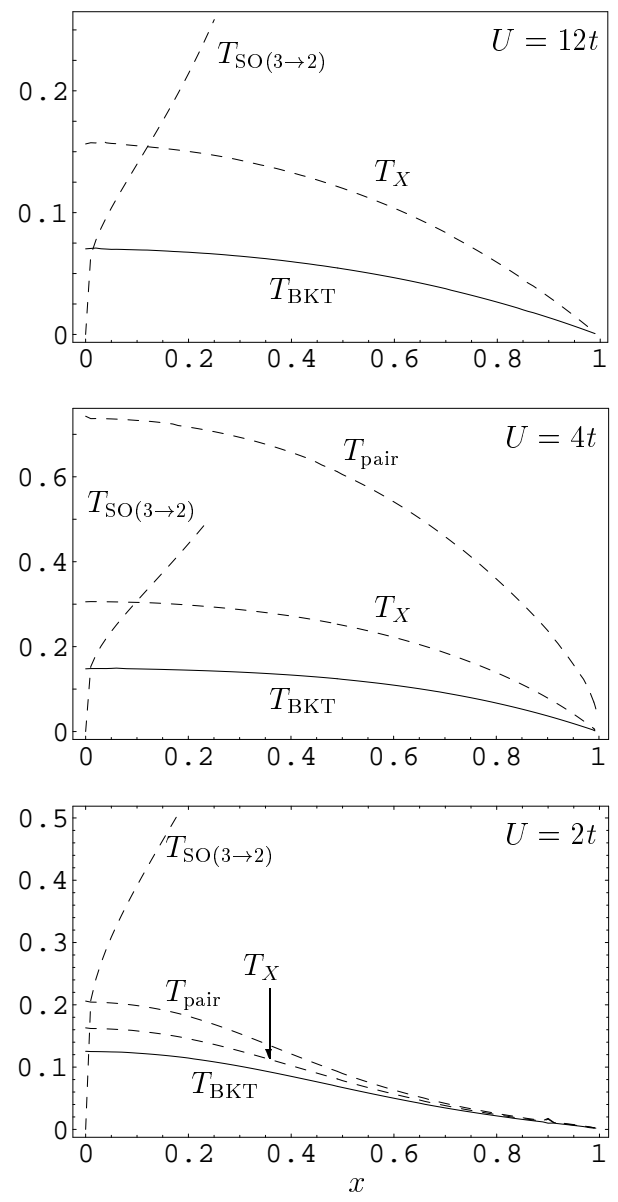

FIG. 8: $T_{\mathrm{BKT}}, T_{\mathrm{SO}(3 \rightarrow 2)}, T_{X}$ and $T_{\text {pair }} v s$ doping $x$ for $U=$ $12 t, 4 t$ and $2 t$. For $U=12 t, T_{\text {pair }}$, which is nearly a vertical line around $x=1$ on the scale of the figure, is not shown.

This corresponds to a regime of fluctuations with $\mathrm{SO}(2)$ symmetry which eventually drive a BKT phase transition at the temperature $T_{\mathrm{BKT}}$. Once the $\mathrm{SO}(2)$ regime is reached, the BKT phase transition occurs rapidly, so that $T_{\mathrm{BKT}}$ can be estimated by $T_{\mathrm{SO}(3 \rightarrow 2)}$ (see Sec. IIIB). Therefore, the true transition temperature is estimated as $\min \left(T_{\mathrm{BKT}}, T_{\mathrm{SO}(3 \rightarrow 2)}\right)$, where $T_{\mathrm{BKT}}$ is the transition temperature obtained in Sec. IIE i.e. by neglecting the $\mathbf{q}=\mathbf{Q}$ charge fluctuations. The resulting phase diagram is shown in Fig. 9. In the intermediate and strongcoupling regimes (i.e. for $U \gtrsim 4 t$ ), we clearly identify a $\mathrm{RC}$ regime above the $\mathrm{BKT}$ superconducting phase and an incoherent-pair regime at higher temperatures. We believe the persistence of the incoherent-pair regime at weak-coupling (see Fig. 9 for $U=2 t$ ) to be likely an artifact of our simple estimate of $T_{\text {pair }}$. Due to many-body effects not taken into account in our approach, $T_{\text {pair }}$ is reduced with respect to the HF transition temperature ${ }^{52}$ We expect $T_{\text {pair }} \simeq T_{X}$ at weak coupling and thus the disappearance of the incoherent-pair regime. We also observe that the $\mathrm{RC}$ regime with $\mathrm{SO}(3)$ fluctuations extends to higher doping at strong coupling. Fig. 10 shows the phase diagram as a function of $U$ for $x=0.1,0.5$ and 

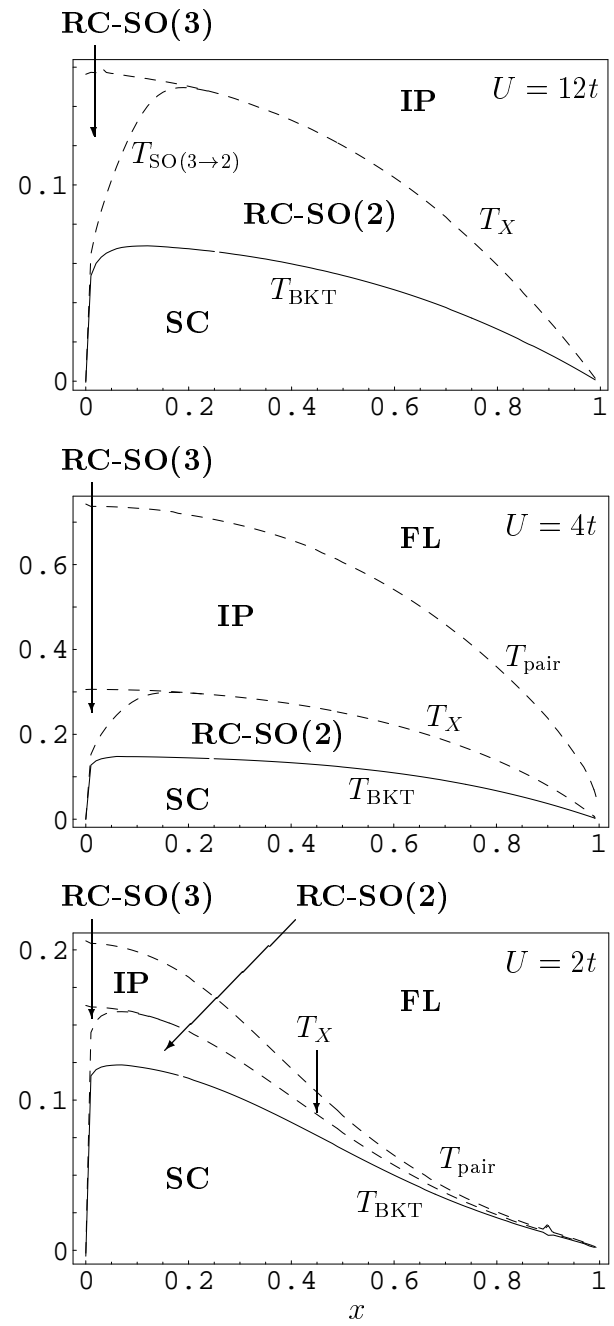

FIG. 9: Phase diagram of the 2D attractive Hubbard model vs doping $x$ for $U=12 t, 4 t$ and $2 t$. SC: superconducting phase (algebraic order). $\mathrm{RC}-\mathrm{SO}(2)$ : renormalized classical regime with fluctuations of $\mathrm{SO}(2)$ symmetry. RC-SO(3): RC regime where the fluctuations exhibit an effective $\mathrm{SO}(3)$ symmetry due to the presence of strong $\mathbf{q}=\mathbf{Q}$ charge fluctuations. FL: Fermi liquid. The incoherent-pair regime (IP) corresponds to the formation of Cooper (local) pairs at weak (strong) coupling, without short-range SC order. Superconducting longrange order sets in at $T=0$.

0.9 .

Away from half-filling, the BKT phase transition temperature derived from the criterion $T_{\mathrm{BKT}} \propto \rho_{s}^{0}\left(T_{\mathrm{BKT}}\right)$, with $\rho_{s}^{0}$ given by (60), was also discussed in Refs. 43, 44 45 46. Good quantitative agreement with Quantum Monte-Carlo simulations was obtained $\stackrel{45}{U}$ Our estimation of the crossover temperature $T_{X}$ for $U=4 t$ also agrees with the Quantum Monte-Carlo simulations of Ref. 51 .

In the weak-coupling limit, we expect a pseudogap to appear in the density of states and the spectral function $A(\mathbf{k}, \omega)=-\pi^{-1} \operatorname{Im} G(\mathbf{k}, \omega)$ below the crossover temperature $T_{X}[G(\mathbf{k}, \omega)$ is the single-particle Green's function] $\stackrel{51.52 .53}{5}$ This pseudogap results from strong scat-
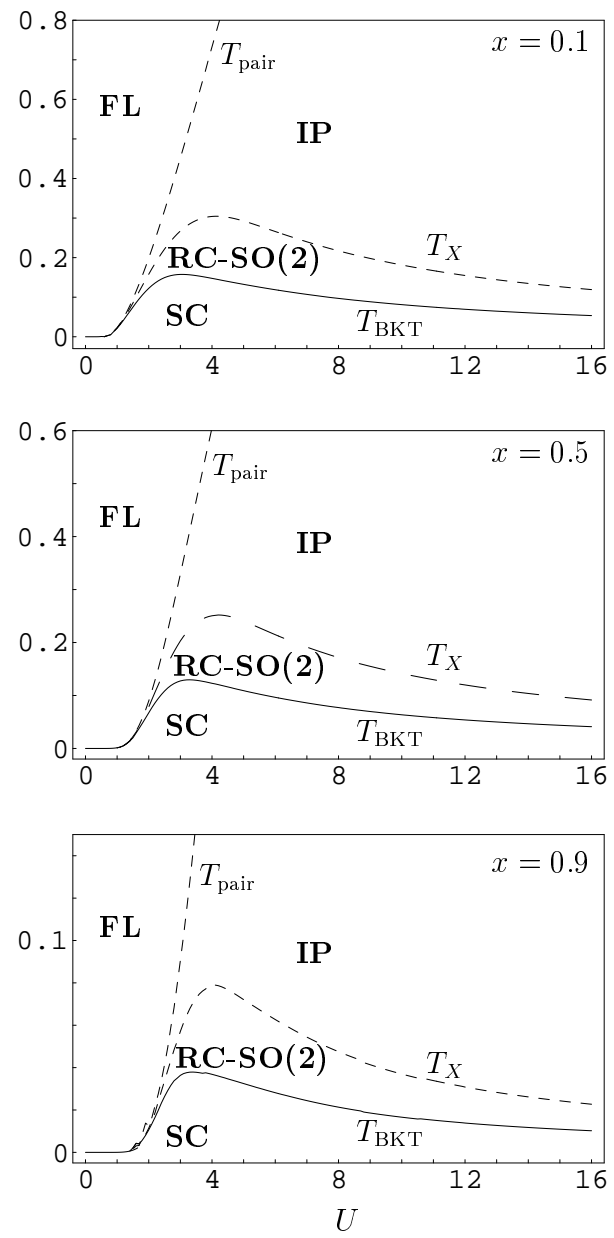

FIG. 10: Phase diagram of the $2 \mathrm{D}$ attractive Hubbard model vs $U$ for $x=0.1,0.5$ and 0.9 .

tering of particles on collective fluctuations in the $\mathrm{RC}$ regime. ${ }^{51.52}$ In the BCS limit, the pseudogap region is small, except in the vicinity of half-filling where an $\mathrm{SO}(3)$ $\mathrm{RC}$ regime is observed over a wide temperature range $\left(0 \leq T \lesssim T_{X}\right)$ [see Fig. 9.54 As the interaction strength increases, the weak-coupling pseudogap should progressively evolves into a strong-coupling gap due to the formation of local singlet pairs when $T \lesssim T_{\text {pair. }}$. Pseudogaps in the 2D attractive Hubbard model have been seen both in Quantum Monte Carlo simulations ${ }^{51.53 .54}$ and analytical approaches ${ }^{1.51 .52 .55 .56 .57}$ At half-filling, the transformation of the weak-coupling pseudogap into the strongcoupling gap has been studied in Refs. 23 24.

\section{NEAR HALF-FILLING}

In this section, we calculate the crossover temperature $T_{\mathrm{SO}(3 \rightarrow 2)}$ introduced in Sec. IIF The approach of Sec. II holds only when $\mathbf{q}=\mathbf{Q}$ charge fluctuations (in the attractive model) are weak for all temperatures below $T_{X}$. In the repulsive model, this corresponds to the limit where 
the magnetic field $h_{0}$ is strong enough to suppress fluctuations of the $S^{z}$ component of the spin density. Near half-filling, $\mathbf{q}=\mathbf{Q}$ charge fluctuations are suppressed only at very low temperature. We therefore expect a $\mathrm{RC}$ regime with $\mathrm{SO}(3)$ symmetry in the temperature range $T_{\mathrm{SO}(3 \rightarrow 2)} \leq T \leq T_{X}$ followed by a $\mathrm{RC}$ regime with $\mathrm{SO}(2)$ symmetry at lower temperature $\left(T_{\mathrm{BKT}} \leq T \leq T_{\mathrm{SO}(3 \rightarrow 2)}\right)$.

In the repulsive model, a weak magnetic field $\mathbf{h}_{0}=$ $h_{0} \hat{\mathbf{z}}$ can be treated perturbatively. When it vanishes, the low-energy effective action for spin fluctuations is a NL $\sigma \mathrm{M} \cdot{ }^{23.24 .28}$ From general arguments,, 58 we expect the magnetic field to modify the kinetic term of the NL $\sigma \mathrm{M}$, $\dot{\mathbf{n}}_{\mathbf{r}}^{2} \rightarrow\left(\dot{\mathbf{n}}_{\mathbf{r}}-2 i \mathbf{h}_{0} \times \mathbf{n}_{\mathbf{r}}\right)^{2}$, as obtained in the Heisenberg limit ${ }^{59}$ [Here $\mathbf{n}\left(\mathbf{n}^{2}=1\right)$ is the Néel field describing low-energy AF fluctuations.] We show below that this is indeed correct. We consider the low-temperature limit $T \ll T_{X}$ where the coefficients of the effective action can be evaluated in the zero-temperature limit.

Following Ref. 24, we introduce only one auxiliary field $\left(m_{\mathbf{r}}\right)$ to decouple the spin term $\left(c_{\mathbf{r}}^{\dagger} \boldsymbol{\sigma} \cdot \boldsymbol{\Omega}_{\mathbf{r}} c_{\mathbf{r}}\right)^{2}$ in (9) ${ }^{60}$ The action then reads

$$
S=\int_{0}^{\beta} d \tau \sum_{\mathbf{r}}\left[c_{\mathbf{r}}^{\dagger}\left(\partial_{\tau}-\hat{t}-h_{0} \sigma^{z}-m_{\mathbf{r}} \boldsymbol{\sigma} \cdot \boldsymbol{\Omega}_{\mathbf{r}}\right) c_{\mathbf{r}}+\frac{m_{\mathbf{r}}^{2}}{U}\right]
$$

At the HF level, neglecting the magnetic field, we have $m_{\mathbf{r}}=m_{0}$ and $\boldsymbol{\Omega}_{\mathbf{r}}^{\mathrm{cl}}=(-1)^{\mathbf{r}} \hat{\mathbf{z}}$. We choose the AF magnetization along the $z$ axis as in Ref. 24. The saddlepoint equation reads $2 m_{0} / U=(-1)^{\mathbf{r}}\left\langle c_{\mathbf{r}}^{\dagger} \sigma^{z} c_{\mathbf{r}}\right\rangle$. Following Haldane, ${ }^{38.61}$ in the presence of AF short-range order $\left(T \ll T_{X}\right)$, we write

$$
\boldsymbol{\Omega}_{\mathbf{r}}=(-1)^{\mathbf{r}} \mathbf{n}_{\mathbf{r}} \sqrt{1-\mathbf{L}_{\mathbf{r}}^{2}}+\mathbf{L}_{\mathbf{r}}
$$

$\mathbf{n}_{\mathbf{r}}$ is the slowly varying Néel field, whereas $\mathbf{L}_{\mathbf{r}}$ is a canting vector, orthogonal to $\mathbf{n}_{\mathbf{r}}$, taking account of local ferromagnetic fluctuations. We assume $\mathbf{L}_{\mathbf{r}}$ to be small, which clearly restricts the validity of this approach to weak magnetic fields. As in Sec. III we introduce a new field $\phi$ defined by $c_{\mathbf{r}}=R_{\mathbf{r}} \phi_{\mathbf{r}}$ where $R_{\mathbf{r}}$ is a time- and site-dependent $\mathrm{SU}(2) / \mathrm{U}(1)$ matrix satisfying

$$
R_{\mathbf{r}} \sigma^{z} R_{\mathbf{r}}^{\dagger}=\boldsymbol{\sigma} \cdot \mathbf{n}_{\mathbf{r}}
$$

$\mathcal{R}_{\mathbf{r}}$, the $\mathrm{SO}(3)$ element associated to $R_{\mathbf{r}}$, maps $\hat{\mathbf{z}}$ onto $\mathbf{n}_{\mathbf{r}}$. We also define the rotated canting field $\mathbf{l}_{\mathbf{r}}=\mathcal{R}_{\mathbf{r}}^{-1} \mathbf{L}_{\mathbf{r}}$. Given that $\mathcal{R}_{\mathbf{r}}^{-1} \mathbf{n}_{\mathbf{r}}=\hat{\mathbf{z}}$ and $\mathbf{L}_{\mathbf{r}} \perp \mathbf{n}_{\mathbf{r}}$, the $\mathbf{l}_{\mathbf{r}}$ vector lies in the $(x-y)$ plane.

In order to express the action in terms of the $\phi$ field, it is convenient to make use of the $\mathrm{SU}(2)$ gauge field $A_{\mu \mathbf{r}}=\sum_{\nu=x, y, z} A_{\mu \mathbf{r}}^{\nu} \sigma^{\nu}$ defined as

$$
\begin{aligned}
& A_{0 \mathbf{r}}=-R_{\mathbf{r}}^{\dagger} \partial_{\tau} R_{\mathbf{r}}, \\
& A_{\mu \mathbf{r}}=i R_{\mathbf{r}}^{\dagger} \partial_{\mu} R_{\mathbf{r}} \quad(\mu=x, y) .
\end{aligned}
$$

Since the gauge field is of order $O\left(\partial_{\mu}\right)$, we can expand the action with respect to $1, A_{\mu}, h_{0}$ and $\delta m$. To second order, we obtain

$$
\begin{aligned}
S= & S_{\mathrm{HF}}+S_{p}+S_{l}+S_{d}+S_{l^{2}}+S_{h_{0}}+S_{\delta m} \\
& +\int_{0}^{\beta} d \tau \sum_{\mathbf{r}} \frac{\delta m_{\mathbf{r}}^{2}+2 m_{0} \delta m_{\mathbf{r}}}{U}, \\
S_{\mathrm{p}}= & -\int_{0}^{\beta} d \tau \sum_{\substack{\mu=0, x, y \\
\nu=x, y \\
\mathbf{r}, z}} j_{\mu \mathbf{r}}^{\nu} A_{\mu \mathbf{r}}^{\nu}, \\
S_{\mathrm{d}}= & \frac{t}{2} \int_{0}^{\beta} d \tau \sum_{\substack{\mu=x, y \\
\nu=x, y}} A_{\mu \mathbf{r}}^{\nu} \phi_{\mathbf{r}}^{\dagger} \cos \left(-i \partial_{\mu}\right) \phi_{\mathbf{r}}+\text { c.c. }, \\
S_{l}= & -m_{0} \int_{0}^{\beta} d \tau \sum_{\nu=x, y} l_{\mathbf{r}}^{\nu} j_{0 \mathbf{r}}^{\nu}, \\
S_{l^{2}}= & \frac{m_{0}}{2} \int_{0}^{\beta} d \tau \sum_{\mathbf{r}}(-1)^{\mathbf{r}} \mathbf{l}_{\mathbf{r}}^{2} j_{0 \mathbf{r}}^{z}, \\
S_{h_{0}}= & -h_{0} \int_{0}^{\beta} d \tau \sum_{\mathbf{r}} \phi_{\mathbf{r}}^{\dagger} R_{\mathbf{r}}^{\dagger} \sigma^{z} R_{\mathbf{r}} \phi_{\mathbf{r}}, \\
S_{\delta m}= & -\int_{0}^{\beta} d \tau \sum_{\mathbf{r}}(-1)^{\mathbf{r}} \delta m_{\mathbf{r}} j_{0 \mathbf{r}}^{z},
\end{aligned}
$$

where the spin-density current $j_{\mu \mathbf{r}}^{\nu}$ is defined in (A2). For $h_{0}=0, S$ reduces to the action derived in Ref. 24 . $S_{\mathrm{HF}}$ is the $\mathrm{HF}$ action. $S_{p}$ and $S_{d}$ are paramagnetic and diamagnetic terms, respectively. $S_{l}$ and $S_{l^{2}}$ are first-order and second-order corrections in 1. $S_{\delta m}$ is the contribution due to amplitude fluctuations. 62

The effective action $S[\mathbf{n}, \mathbf{L}, \delta m]$ is obtained by integrating out the fermions. To second order in $A_{\mu}, 1, \delta m$ and $h_{0}$, one finds

$$
\begin{aligned}
S[\mathbf{n}, \mathbf{L}, \delta m]= & S\left[\mathbf{n}, \mathbf{L} ; h_{0}=\delta m=0\right]+\left\langle S_{p}+S_{h_{0}}+S_{\delta m}\right\rangle \\
& -\frac{1}{2}\left\langle\left(S_{h_{0}}+S_{\delta m}\right)^{2}\right. \\
& \left.+2\left(S_{h_{0}}+S_{\delta m}\right)\left(S_{p}+S_{l}\right)\right\rangle_{c} \\
& +\int_{0}^{\beta} d \tau \sum_{\mathbf{r}} \frac{\delta m_{\mathbf{r}}^{2}+2 m_{0} \delta m_{\mathbf{r}}}{U},
\end{aligned}
$$

where the averages $\langle\cdots\rangle$ are taken with the HF action. $S\left[\mathbf{n}, \mathbf{L} ; h_{0}=\delta m=0\right]$ is the action with no magnetic field (and no amplitude fluctuations) and was derived in Ref. 24. $\left\langle S_{p}\right\rangle$ is a Berry phase term. It was ignored in Ref. 24, since it does not play any role in the $\mathrm{RC}$ regime of the $h_{0}=0 \mathrm{NL} \sigma \mathrm{M}$. In order to calculate the HF averages, we write

$$
S_{h_{0}}=-h_{0} \int_{0}^{\beta} d \tau \sum_{\substack{\mathbf{r} \\ \nu=x, y, z}} B_{0 \mathbf{r}}^{\nu} j_{0 \mathbf{r}}^{\nu}
$$

where $B_{0 \mathbf{r}}^{\nu}$ is defined by $R_{\mathbf{r}}^{\dagger} \sigma^{z} R_{\mathbf{r}}=\sum_{\nu=x, y, z} B_{0 \mathbf{r}}^{\nu} \sigma^{\nu} . A_{\mu \mathbf{r}}^{\nu}$ and $B_{0 \mathbf{r}}^{\nu}$ are calculated using

$$
R_{\mathbf{r}}=\left(\begin{array}{rr}
\cos \left(\frac{\theta_{\mathbf{r}}}{2}\right) e^{-\frac{i}{2}\left(\varphi_{\mathbf{r}}+\psi_{\mathbf{r}}\right)} & -\sin \left(\frac{\theta_{\mathbf{r}}}{2}\right) e^{-\frac{i}{2}\left(\varphi_{\mathbf{r}}-\psi_{\mathbf{r}}\right)} \\
\sin \left(\frac{\theta_{\mathbf{r}}}{2}\right) e^{\frac{i}{2}\left(\varphi_{\mathbf{r}}-\psi_{\mathbf{r}}\right)} & \cos \left(\frac{\theta_{\mathbf{r}}}{2}\right) e^{\frac{i}{2}\left(\varphi_{\mathbf{r}}+\psi_{\mathbf{r}}\right)}
\end{array}\right) .
$$


Here we define $\mathbf{n}_{\mathbf{r}}$ by its polar and azimuthal angles $\theta_{\mathbf{r}}$ and $\varphi_{\mathbf{r}}$. The angle $\psi_{\mathbf{r}}$ comes from the $\mathrm{U}(1)$ gauge freedom in the definition of $R_{\mathbf{r}}$. We obtain

$$
\begin{aligned}
A_{0 \mathbf{r}}^{x} & =\frac{i}{2} \dot{\theta}_{\mathbf{r}} \sin \psi_{\mathbf{r}}-\frac{i}{2} \dot{\varphi}_{\mathbf{r}} \sin \theta_{\mathbf{r}} \cos \psi_{\mathbf{r}}, \\
A_{0 \mathbf{r}}^{y} & =\frac{i}{2} \dot{\theta}_{\mathbf{r}} \cos \psi_{\mathbf{r}}+\frac{i}{2} \dot{\varphi}_{\mathbf{r}} \sin \theta_{\mathbf{r}} \sin \psi_{\mathbf{r}}, \\
A_{0 \mathbf{r}}^{z} & =\frac{i}{2} \dot{\varphi}_{\mathbf{r}} \cos \theta_{\mathbf{r}}+\frac{i}{2} \dot{\psi}_{\mathbf{r}}, \\
B_{0 \mathbf{r}}^{x} & =-\sin \theta_{\mathbf{r}} \cos \psi_{\mathbf{r}}, \\
B_{0 \mathbf{r}}^{y} & =\sin \theta_{\mathbf{r}} \sin \psi_{\mathbf{r}}, \\
B_{0 \mathbf{r}}^{z} & =\cos \theta_{\mathbf{r}} .
\end{aligned}
$$

$A_{\mu \mathbf{r}}^{\nu}(\mu \neq 0)$ is obtained from $A_{0 \mathbf{r}}^{\nu}$ with the replacement $\partial_{\tau} \rightarrow-i \partial_{\mu}$.

We find (see Appendix E)

$$
\begin{aligned}
\left\langle S_{p}\right\rangle & =-i \frac{m_{0}}{U} \int_{0}^{\beta} d \tau \sum_{\mathbf{r}}(-1)^{\mathbf{r}}\left(\dot{\varphi}_{\mathbf{r}} \cos \theta_{\mathbf{r}}+\dot{\psi}_{\mathbf{r}}\right), \\
\left\langle S_{h_{0}}\right\rangle & =0 \\
\left\langle S_{h_{0}}^{2}\right\rangle & =\Pi_{00}^{x x} \int_{0}^{\beta} d \tau \sum_{\mathbf{r}}\left(\mathbf{h}_{0} \times \mathbf{n}_{\mathbf{r}}\right)^{2} \\
\left\langle S_{h_{0}} S_{p}\right\rangle & =\frac{i}{2} \Pi_{00}^{x x} \int_{0}^{\beta} d \tau \sum_{\mathbf{r}} \mathbf{h}_{0} \cdot\left(\mathbf{n}_{\mathbf{r}} \times \dot{\mathbf{n}}_{\mathbf{r}}\right) \\
\left\langle S_{h_{0}} S_{l}\right\rangle & =m_{0} \Pi_{00}^{x x} \int_{0}^{\beta} d \tau \sum_{\mathbf{r}} \mathbf{h}_{0} \cdot \mathbf{L}_{\mathbf{r}},
\end{aligned}
$$

and $\left\langle S_{h_{0}} S_{\delta m}\right\rangle=\left\langle S_{\delta m} S_{p}\right\rangle=\left\langle S_{\delta m} S_{l}\right\rangle=0$. There is therefore no coupling between amplitude and direction fluctuations in the limit of a small magnetic field. The HF correlation function $\Pi_{00}^{x x} \equiv \Pi_{00}^{x x}(0,0)$ is given in Appendix A2 Using the result of Ref. 24 for $S\left[\mathbf{n}, \mathbf{L} ; h_{0}=\delta m=0\right]$, one has

$$
\begin{aligned}
S[\mathbf{n}, \mathbf{L}]= & \int_{0}^{\beta} d \tau \sum_{\mathbf{r}}\left[\frac{\langle-K\rangle}{16}\left(\nabla \mathbf{n}_{\mathbf{r}}\right)^{2}\right. \\
& +\frac{\Pi_{00}^{x x}}{8}\left(\dot{\mathbf{n}}_{\mathbf{r}}-2 i \mathbf{h}_{0} \times \mathbf{n}_{\mathbf{r}}\right)^{2} \\
& +m_{0}^{2}\left(\frac{1}{U}-\frac{\Pi_{00}^{x x}}{2}\right) \mathbf{L}_{\mathbf{r}}^{2} \\
& \left.-\frac{i}{2} m_{0} \Pi_{00}^{x x} \mathbf{L}_{\mathbf{r}} \cdot\left(\mathbf{n}_{\mathbf{r}} \times \dot{\mathbf{n}}_{\mathbf{r}}-2 i \mathbf{h}_{0}\right)\right] \\
& +S_{B}[\mathbf{n}],
\end{aligned}
$$

where we have taken the continuum limit in real space. $\langle K\rangle$ is the mean kinetic energy in the HF state (for $\left.h_{0}=0\right)$. We denote the Berry phase term $\left\langle S_{p}\right\rangle$ by $S_{B}[\mathbf{n}]$. Integrating out the $\mathbf{L}$ field with the constraint $\mathbf{L}_{\mathbf{r}} \perp \mathbf{n}_{\mathbf{r}}$, we finally obtain

$$
\begin{aligned}
S[\mathbf{n}]= & \frac{\bar{\rho}_{s}^{0}}{2} \int_{0}^{\beta} d \tau \int d^{2} r\left[\left(\nabla \mathbf{n}_{\mathbf{r}}\right)^{2}+\frac{\left(\dot{\mathbf{n}}_{\mathbf{r}}-2 i \mathbf{h}_{0} \times \mathbf{n}_{\mathbf{r}}\right)^{2}}{\bar{c}^{2}}\right] \\
& +S_{B}[\mathbf{n}],
\end{aligned}
$$

where

$$
\begin{aligned}
& \bar{\rho}_{s}^{0}=\frac{\langle-K\rangle}{8} \\
& \bar{c}^{2}=\frac{\langle-K\rangle}{2}\left(\frac{1}{\Pi_{00}^{x x}}-\frac{U}{2}\right) .
\end{aligned}
$$

$\bar{\rho}_{s}^{0}$ and $\bar{c}$ are the spin stiffness and the spin-wave velocity in the absence of field $\left(h_{0}=0\right.$, i.e. at half-filling in the attractive model). It can be checked analytically that the expression of $\bar{c}$ agrees with (53) evaluated at $h_{0}=0$. The action (75) is valid in the hydrodynamic regime defined by the momentum-space cutoff $\Lambda \sim \min \left(1,2 m_{0} / \bar{c}\right)$. In the strong-coupling limit, $\bar{c}=\sqrt{2} J$ and $\bar{\rho}_{s}^{0}=J / 4$, we recover the NL $\sigma \mathrm{M}$ obtained from the Heisenberg model in a magnetic field ${ }^{59}$ The crossover temperature $T_{X}$ can be obtained from the criterion $\xi \sim 1$, where $\xi$ is the $\mathrm{AF}$ correlation length deduced from the $\mathrm{NL} \sigma \mathrm{M}{ }^{24}$ However, since $T_{X}$ is weakly doping dependent near half-filling, we can also consider the estimate obtained in Sec. IIF [Eq. [63] ].

\section{A. Low-temperature limit}

In the low-temperature limit, one expects to recover the results obtained in Sec. III Let us first consider the $\mathrm{NL} \sigma \mathrm{M}$ (75) within a static saddle-point approximation where the Néel field $\mathbf{n}_{\mathbf{r}}^{\text {cl }}$ lies in the $(x, y)$ plane. The classical action reads

$$
S_{\mathrm{cl}}=-N \beta \frac{2 \bar{\rho}_{s}^{0} h_{0}^{2}}{\bar{c}^{2}} .
$$

The magnetic field $h_{0}$ is determined by the condition $\left\langle c_{\mathbf{r}}^{\dagger} \sigma^{z} c_{\mathbf{r}}\right\rangle=(N \beta)^{-1} \partial_{h_{0}} \ln Z=-x$. From (77), we deduce

$$
h_{0}=-x \frac{\bar{c}^{2}}{4 \bar{\rho}_{s}^{0}} .
$$

The chemical potential $\mu \equiv h_{0}-U / 2$ obtained from (78) is in very good agreement with the result of Sec. II for $x \lesssim 0.2$ (Fig. 111). In the strong-coupling limit, $\bar{c}=\sqrt{2} J$ and $\bar{\rho}_{s}^{0}=J / 4$, we find $h_{0}=-2 J x$ as in Sec. I

Let us now consider fluctuations about the Néel state defined by $\mathbf{n}_{\mathbf{r}}^{\mathrm{cl}}{ }^{63}$ We have shown in Sec. IIC that the phase of the superconducting order parameter is obtained from $\Theta_{\mathbf{r}}=-\arctan \left(\Omega_{\mathbf{r}}^{y} / \Omega_{\mathbf{r}}^{x}\right)+\mathbf{Q} \cdot \mathbf{r}$ [Eq. (D6)]. From (65), we then deduce $\Theta_{\mathbf{r}}=-\varphi_{\mathbf{r}}$. It is also clear that the $\theta$ field is related to the $\mathbf{q}=\mathbf{Q}$ charge fluctuations. In the low-temperature limit $\left|n_{\mathbf{r}}^{z}\right| \ll 1$. The low-energy effective action $S[\Theta, \theta]$ is obtained by assum$\operatorname{ing}\left|\nabla \Theta_{\mathbf{r}}\right|,\left|\dot{\Theta}_{\mathbf{r}}\right|,\left|\nabla \theta_{\mathbf{r}}\right|,\left|\dot{\theta}_{\mathbf{r}}\right|,\left|\theta_{\mathbf{r}}-\pi / 2\right| \ll 1$. For the calculation of the Berry phase term $S_{B}[\mathbf{n}]$, we choose $\psi_{\mathbf{r}}=$ $(-1)^{\mathbf{r}} \varphi_{\mathbf{r}} U /\left(2 m_{0}\right)$. As in Sec. III this gauge choice ensures that electromagnetic gauge invariance is satisfied ${ }^{34}$ This 


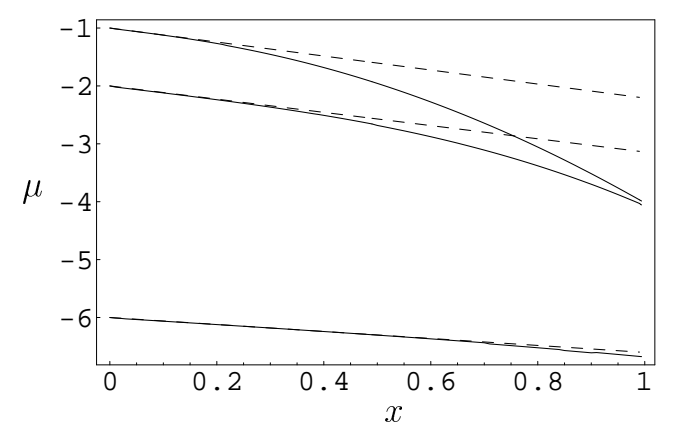

FIG. 11: Zero-temperature chemical potential $\mu=h_{0}-U / 2$ $v s$ doping $x$ for $U=2 t, 4 t$ and $12 t$. The dashed lines show the result obtained from Eq. (78). The solid lines reproduce the results obtained by solving Eqs. 22 23] [see Fig. 2].

gives $S[\Theta, \theta]=S_{\mathrm{cl}}+S[\Theta]+S[\theta]$ where

$$
\begin{aligned}
S[\Theta]= & \frac{\bar{\rho}_{s}^{0}}{2} \int_{0}^{\beta} d \tau \int d^{2} r\left[\left(\nabla \Theta_{\mathbf{r}}\right)^{2}+\frac{\dot{\Theta}_{\mathbf{r}}^{2}}{\bar{c}^{2}}\right] \\
& +\frac{i}{2} \rho_{0} \int_{0}^{\beta} d \tau \int d^{2} \dot{\Theta}_{\mathbf{r}}, \\
S[\theta]= & \frac{\bar{\rho}_{s}^{0}}{2} \int_{0}^{\beta} d \tau \int d^{2} r\left[\left(\nabla \theta_{\mathbf{r}}\right)^{2}+\frac{\dot{\theta}_{\mathbf{r}}^{2}}{\bar{c}^{2}}\right. \\
& \left.+\frac{\left(2 h_{0}\right)^{2}}{\bar{c}^{2}}\left(\theta_{\mathbf{r}}-\frac{\pi}{2}\right)^{2}\right] .
\end{aligned}
$$

$S[\Theta]$ corresponds to the $h_{0}=0$ limit of the phase-only action (52) derived in Sec. III $S[\theta]$ describes $\mathbf{q}=\mathbf{Q}$ charge fluctuations with a gap $2\left|h_{0}\right|$. These fluctuations were not considered in Sec. II. We show in the next section that they play an important role when $\left|h_{0}\right|$ is small, i.e. near half-filling in the attractive model.

\section{B. High-temperature limit: $\mathrm{SO}(3) \rightarrow \mathrm{SO}(2)$ crossover and BKT transition}

The preceding results are valid at low temperatures when fluctuations of $\theta_{\mathbf{r}}$ are small. In this regime, the $\mathrm{SO}(3)$ rotation symmetry is broken by the magnetic field. At higher temperatures, the gapped mode will be thermally excited thus restoring the $\mathrm{SO}(3)$ spin-rotation symmetry. In this section, we determine the crossover temperature $T_{\mathrm{SO}(3 \rightarrow 2)}$ separating these two regimes and the BKT transition temperature $T_{\mathrm{BKT}} \leq T_{\mathrm{SO}(3 \rightarrow 2)}$.

The $\mathrm{SO}(3) \rightarrow \mathrm{SO}(2)$ crossover can be understood from renormalization group (RG) arguments. Following the standard procedure,,$\stackrel{64}{=}$ we write the Néel field $\mathbf{n}_{\mathbf{r}}$ as $((1-$ $\left.\left.\boldsymbol{\Pi}_{\mathbf{r}}{ }^{2}\right)^{1 / 2}, \boldsymbol{\Pi}_{\mathbf{r}}\right), \boldsymbol{\Pi}_{\mathbf{r}}=\left(\Pi_{\mathbf{r}}^{y}, \Pi_{\mathbf{r}}^{z}\right)$, with $\left|\boldsymbol{\Pi}_{\mathbf{r}}\right| \ll 1$. This yields the spin-wave action

$$
S=\frac{\bar{\rho}_{s}^{0}}{2} \int_{0}^{\beta} d \tau \int d^{2} r\left[\left(\nabla \Pi_{\mathbf{r}}\right)^{2}+\frac{\dot{\boldsymbol{\Pi}}_{\mathbf{r}}^{2}}{\bar{c}^{2}}+\frac{4 h_{0}^{2}}{\bar{c}^{2}} \Pi_{\mathbf{r}}^{z 2}\right]+S_{1},
$$

with momenta bounded by the NL $\sigma \mathrm{M}$ cutoff $\Lambda$. In agreement with Sec. IIIA we find a gap in the (bare) propagator of the $\Pi^{z}$ field. The interaction part $S_{1}$ of the action is evaluated at $h_{0}=0$. RG equations are obtained by integrating out degrees of freedom with momenta between $\Lambda$ and $\Lambda e^{-d l}$ and rescaling momenta, energies and fields ${ }^{64}$ At the beginning of the RG procedure, we can ignore the gap in the fluctuations of $\Pi^{z}$ and treat the magnetic field perturbatively. We then obtain the usual RG equations of the $\mathrm{SO}(3) \mathrm{NL} \sigma \mathrm{M}\left(h_{0}=0\right)$ for the dimensionless coupling constants $g=\bar{c} \Lambda / \bar{\rho}_{s}^{0}$ and $t=T / \bar{\rho}_{s}^{0}$, together with the flow equation $d h_{0} / d l=h_{0}$. The RG flow should be stopped when we reach the strong-coupling regime of the $\mathrm{SO}(3) \mathrm{NL} \sigma \mathrm{M}(t(l) \sim 1)$ or when the magnetic field cannot be treated perturbatively anymore $\left(\left|2 h_{0}(l)\right| \sim \bar{c} \Lambda\right.$ ). Introducing the characteristic length $\Lambda^{-1} e^{l}$, the former condition defines the $\mathrm{SO}(3) \mathrm{AF}$ correlation length $\xi$ while the latter defines the magnetic length $\xi_{h_{0}}=c /\left|2 h_{0}\right|$. We are therefore left to consider two different cases. If $\xi \lesssim \xi_{h_{0}}, t(l) \sim 1$ occurs before $\left|2 h_{0}(l)\right| \sim \bar{c} \Lambda$. The system is then disordered by $\mathrm{SO}(3)$ fluctuations before reaching the $\mathrm{SO}(2)$ regime. This will occur above the crossover temperature $T_{\mathrm{SO}(3 \rightarrow 2)}$ defined by $\xi\left(T_{\mathrm{SO}(3 \rightarrow 2)}\right) \sim \xi_{h_{0}}{ }^{65}$ If $\xi_{h_{0}} \lesssim \xi$, i.e. $T \lesssim T_{\mathrm{SO}(3 \rightarrow 2)}$, an $\mathrm{SO}(3) \rightarrow \mathrm{SO}(2)$ crossover takes place. For $\left|2 h_{0}(l)\right|>\bar{c} \Lambda$, fluctuations of $\Pi^{z}$ are suppressed, and only fluctuations of $\Pi^{y}$ survive. This regime is described by an $\mathrm{SO}(2) \mathrm{NL} \sigma \mathrm{M}$ with coupling constants $t\left(l_{h_{0}}\right)$ and $g\left(l_{h_{0}}\right)$ where $l_{h_{0}}$ is defined by $\left|2 h_{0}\left(l_{h_{0}}\right)\right| \sim \bar{c} \Lambda$. The very existence of the $\mathrm{SO}(2)$ regime implies $t\left(l_{h_{0}}\right) \lesssim 1$. The latter inequality is also approximately the condition for the $\mathrm{SO}(2) \mathrm{NL} \sigma \mathrm{M}$ to be in the low-temperature BKT phase. This implies that the temperature range where the system is disordered by $\mathrm{SO}(2)$ fluctuations is very narrow, so that we can identify the BKT transition temperature with the $\mathrm{SO}(3) \rightarrow \mathrm{SO}(2)$ crossover temperature: $T_{\mathrm{BKT}} \sim T_{\mathrm{SO}(3 \rightarrow 2)}$.

In order to determine $T_{\mathrm{SO}(3 \rightarrow 2)}$, we use the following expression for the $h_{0}=0$ correlation length $\xi:^{64}$

$$
\xi(T)=K \frac{\bar{c}}{\bar{\rho}_{s}} \exp \left(\frac{2 \pi \bar{\rho}_{s}}{T}\right),
$$

where $K \simeq 0.05$ and $\bar{\rho}_{s}$ is the zero-temperature spin stiffness in the Néel state. To estimate $\bar{\rho}_{s}$, we use the oneloop RG result, $\underline{\underline{6}}$

$$
\bar{\rho}_{s}=\bar{\rho}_{s}^{0}\left(1-\frac{\bar{c} \Lambda}{4 \pi \bar{\rho}_{s}^{0}}\right) .
$$

$T_{\mathrm{SO}(3 \rightarrow 2)}$ is therefore given by

$$
T_{\mathrm{SO}(3 \rightarrow 2)}=\frac{2 \pi \bar{\rho}_{s}}{\ln \left(\frac{\bar{\rho}_{s}}{2 K\left|h_{0}\right|}\right)} \simeq \frac{2 \pi \bar{\rho}_{s}}{\ln \left(\frac{10 \bar{\rho}_{s}}{\left|h_{0}\right|}\right)}
$$

where we have taken $K=0.05 . T_{\mathrm{SO}(3 \rightarrow 2)}$, obtained from (84), is shown in Fig. 8

The definition of $T_{\mathrm{SO}(3 \rightarrow 2)}$ is meaningful only below the crossover temperature $T_{X}$ which marks the onset of AF short-range order and defines the temperature range 
where the NL $\sigma \mathrm{M}$ holds. Once $T_{\mathrm{SO}(3 \rightarrow 2)}$ becomes of the order of $T_{X}$, as $\left|h_{0}\right|$ increases, $\mathbf{q}=\mathbf{Q}$ charge fluctuations (in the attractive model) can be ignored. In this regime, the analysis of Sec. II holds.

\section{STRONG-COUPLING LIMIT}

In the strong-coupling limit $U \gg 4 t$, we can directly integrate out the fermions to recover the action $S[\Omega]$ of the Heisenberg model in a magnetic field (Sec. IVA). We can then go beyond the hydrodynamic limit $(\mathbf{q} \rightarrow$ $0)$ considered in Sec. II and obtain the collective mode dispersion over the entire Brillouin zone. When $\left|h_{0}\right|$ is weak enough (i.e. away from the low-density limit in the attractive model), the Heisenberg model reduces to the quantum XY model (Sec.IVB). In the low-density limit, the Heisenberg model allows to recover the usual action of a Bose superfluid, including the terms proportional to $\left(\nabla \rho_{\mathbf{r}}\right)^{2}$ that were omitted in Sec. II and in turn the Gross-Pitaevskii equation (Sec. IVC).

\section{A. Heisenberg model}

We write the action (1112) in terms of the $\phi$ field defined by $c_{\mathbf{r}}=R_{\mathbf{r}} \phi_{\mathbf{r}}$ and $R_{\mathbf{r}} \sigma^{z} R_{\mathbf{r}}^{\dagger}=\boldsymbol{\sigma} \cdot \Omega_{\mathbf{r}}$ :

$$
\begin{aligned}
S= & S_{\mathrm{at}}+\int_{0}^{\beta} d \tau\left[\sum_{\mathbf{r}} \phi_{\mathbf{r}}^{\dagger}\left(R_{\mathbf{r}}^{\dagger} \dot{R}_{\mathbf{r}}-h_{0} R_{\mathbf{r}}^{\dagger} \sigma^{z} R_{\mathbf{r}}\right) \phi_{\mathbf{r}}\right. \\
& \left.-t \sum_{\left\langle\mathbf{r}, \mathbf{r}^{\prime}\right\rangle}\left(\phi_{\mathbf{r}}^{\dagger} R_{\mathbf{r}}^{\dagger} R_{\mathbf{r}^{\prime}} \phi_{\mathbf{r}^{\prime}}+\text { c.c. }\right)\right] \\
S_{\mathrm{at}}= & \int_{0}^{\beta} d \tau \sum_{\mathbf{r}}\left[\phi_{\mathbf{r}}^{\dagger}\left(\partial_{\tau}-i m_{0}^{\mathrm{HS}} \sigma^{z}\right) \phi_{\mathbf{r}}\right]
\end{aligned}
$$

where we neglect spin amplitude fluctuations and $i m_{0}^{\mathrm{HS}}=$ $U / 2$ to leading order in $1 / U$ (see Sec. III). $S_{\text {at }}$ is the action in the atomic limit $(t=0)$. The effective action of the angular variable $\boldsymbol{\Omega}$ is obtained by integrating out the fermions. To lowest order in $t / U$ and $h_{0}$, we obtain

$$
\begin{aligned}
S= & \int_{0}^{\beta} d \tau \sum_{\mathbf{r}}\left[\left\langle\phi_{\mathbf{r}}^{\dagger} R_{\mathbf{r}}^{\dagger} \dot{R}_{\mathbf{r}} \phi_{\mathbf{r}}\right\rangle-h_{0}\left\langle\phi_{\mathbf{r}}^{\dagger} R_{\mathbf{r}}^{\dagger} \sigma^{z} R_{\mathbf{r}} \phi_{\mathbf{r}}\right\rangle\right] \\
& -\frac{1}{2} \int_{0}^{\beta} d \tau d \tau^{\prime} \sum_{\substack{\mathbf{r}_{1}, \mathbf{r}_{2} \\
\mathbf{r}_{1}^{\prime}, \mathbf{r}_{2}^{\prime}}} t_{\mathbf{r}_{1}, \mathbf{r}_{2}} t_{\mathbf{r}_{1}^{\prime}, \mathbf{r}_{2}^{\prime}}\left\langle\left(\phi_{\mathbf{r}_{1}}^{\dagger} R_{\mathbf{r}_{1}}^{\dagger} R_{\mathbf{r}_{2}} \phi_{\mathbf{r}_{2}}\right)_{\tau}\right. \\
& \left.\times\left(\phi_{\mathbf{r}_{1}^{\prime}}^{\dagger} R_{\mathbf{r}_{1}^{\prime}}^{\dagger} R_{\mathbf{r}_{2}^{\prime}} \phi_{\mathbf{r}_{2}^{\prime}}\right)_{\tau^{\prime}}\right\rangle
\end{aligned}
$$

where $t_{\mathbf{r}, \mathbf{r}^{\prime}}$ equals $t$ for $\mathbf{r}, \mathbf{r}^{\prime}$ nearest neighbors and vanishes otherwise. The averages in (87) are taken with the atomic action. They can be easily calculated using the parameterization (71) of $R_{\mathbf{r}}$. One finds

$$
\begin{aligned}
& \left\langle\phi_{\mathbf{r}}^{\dagger} R_{\mathbf{r}}^{\dagger} \dot{R}_{\mathbf{r}} \phi_{\mathbf{r}}\right\rangle=\left\langle\boldsymbol{\Omega}_{\mathbf{r}} \mid \dot{\boldsymbol{\Omega}}_{\mathbf{r}}\right\rangle, \\
& \left\langle\phi_{\mathbf{r}}^{\dagger} R_{\mathbf{r}}^{\dagger} \sigma^{z} R_{\mathbf{r}} \phi_{\mathbf{r}}\right\rangle=\Omega_{\mathbf{r}}^{z}, \\
& \left\langle\left(\phi_{\mathbf{r}_{1}}^{\dagger} R_{\mathbf{r}_{1}}^{\dagger} R_{\mathbf{r}_{2}} \phi_{\mathbf{r}_{2}}\right)_{\tau}\left(\phi_{\mathbf{r}_{1}^{\prime}}^{\dagger} R_{\mathbf{r}_{1}^{\prime}}^{\dagger} R_{\mathbf{r}_{2}^{\prime}} \phi_{\mathbf{r}_{2}^{\prime}}\right)_{\tau^{\prime}}\right\rangle \\
& \simeq \delta_{\mathbf{r}_{1}^{\prime}, \mathbf{r}_{2}} \delta_{\mathbf{r}_{2}^{\prime}, \mathbf{r}_{1}} \delta\left(\tau-\tau^{\prime}\right) \frac{1}{U}\left(1-\boldsymbol{\Omega}_{\mathbf{r}_{1}} \cdot \boldsymbol{\Omega}_{\mathbf{r}_{2}}\right),
\end{aligned}
$$

where we have used $\left(R_{\mathbf{r}}^{\dagger} R_{\mathbf{r}^{\prime}}\right)_{\sigma \bar{\sigma}}\left(R_{\mathbf{r}^{\prime}}^{\dagger} R_{\mathbf{r}}\right)_{\bar{\sigma} \sigma}=\left(1-\Omega_{\mathbf{r}}\right.$. $\left.\boldsymbol{\Omega}_{\mathbf{r}^{\prime}}\right) / 2$. We have introduced the spin $-\frac{1}{2}$ coherent state $\left|\boldsymbol{\Omega}_{\mathbf{r}}\right\rangle=R_{\mathbf{r}}|\uparrow\rangle=\cos \left(\frac{\theta_{\mathbf{r}}}{2}\right) e^{-\frac{i}{2}\left(\varphi_{\mathbf{r}}+\psi_{\mathbf{r}}\right)}|\uparrow\rangle+$ $\sin \left(\frac{\theta_{\mathbf{r}}}{2}\right) e^{\frac{i}{2}\left(\varphi_{\mathbf{r}}-\psi_{\mathbf{r}}\right)}|\downarrow\rangle \cdot 38 \psi_{\mathbf{r}}$ is arbitrary and corresponds to the $\mathrm{U}(1)$ gauge freedom in the definition of the $\mathrm{SU}(2)$ matrix $R_{\mathbf{r}}$. We therefore recover the action

$$
S=\int_{0}^{\beta} d \tau\left\{\sum_{\mathbf{r}}\left[\left\langle\boldsymbol{\Omega}_{\mathbf{r}} \mid \dot{\boldsymbol{\Omega}}_{\mathbf{r}}\right\rangle-\mathbf{h}_{0} \cdot \boldsymbol{\Omega}_{\mathbf{r}}\right]+J \sum_{\left\langle\mathbf{r}, \mathbf{r}^{\prime}\right\rangle} \frac{\boldsymbol{\Omega}_{\mathbf{r}} \cdot \boldsymbol{\Omega}_{\mathbf{r}^{\prime}}}{4}\right\}
$$

of the Heisenberg model in a magnetic field $\mathbf{h}_{0}$.

Consider first the classical ground-state defined by $\boldsymbol{\Omega}_{\mathbf{r}}^{\mathrm{cl}}=(-1)^{\mathbf{r}} \sin \theta_{0} \hat{\mathbf{x}}+\cos \theta_{0} \hat{\mathbf{z}}$. Minimizing the classical action

$$
S_{\mathrm{cl}}=N \beta\left[-h_{0} \cos \theta_{0}+\frac{J}{2} \cos \left(2 \theta_{0}\right)\right]
$$

with respect to $\theta_{0}$, we find

$$
\cos \theta_{0}=\frac{h_{0}}{2 J}
$$

if $\left|h_{0}\right| \leq 2 J$ and $\theta=\pi$ otherwise (for $h_{0} \leq 0$ ). The condition $\left\langle c_{\mathbf{r}}^{\dagger} \sigma^{z} c_{\mathbf{r}}\right\rangle=-x$ translates into $\left\langle\Omega_{\mathbf{r}}^{z}\right\rangle=-x$ (see Appendix D1), i.e. $\cos \theta_{0}=-x$ within the classical approximation. We thus obtain $h_{0}=-2 J x$ as in Sec. II A2

The Heisenberg model allows to obtain the collective excitations of the attractive Hubbard model in the strong-coupling limit without taking the continuum limit. We introduce the variables $p_{\mathbf{r}}=\left(\theta_{\mathbf{r}}-\theta_{0}\right) / 2$ and $q_{\mathbf{r}}=$ $\varphi_{\mathbf{r}}-\mathbf{Q} \cdot \mathbf{r}$ and derive the effective action to quadratic order in $p_{\mathbf{r}}$ and $q_{\mathbf{r}}-q_{\mathbf{r}^{\prime}}$ (for $\mathbf{r}, \mathbf{r}^{\prime}$ first neighbors):

$$
\begin{aligned}
S= & \int_{0}^{\beta} d \tau\left\{\sum _ { \mathbf { r } } \left[\frac{i}{2} \sin \theta_{0}\left(p_{\mathbf{r}} \dot{q}_{\mathbf{r}}-\dot{p}_{\mathbf{r}} q_{\mathbf{r}}\right)+2 h_{0} \cos \theta_{0} p_{\mathbf{r}}^{2}\right.\right. \\
& \left.-\frac{i}{2} \rho_{0} \dot{q}_{\mathbf{r}}\right]+\frac{J}{4} \sum_{\left\langle\mathbf{r}, \mathbf{r}^{\prime}\right\rangle}\left[-2 \cos \left(2 \theta_{0}\right)\left(p_{\mathbf{r}}+p_{\mathbf{r}^{\prime}}\right)^{2}\right. \\
& \left.\left.+\frac{\sin ^{2} \theta_{0}}{2}\left(q_{\mathbf{r}}-q_{\mathbf{r}^{\prime}}\right)^{2}\right]\right\} .
\end{aligned}
$$

The Berry phase term $\left\langle\boldsymbol{\Omega}_{\mathbf{r}} \mid \dot{\boldsymbol{\Omega}}_{\mathbf{r}}\right\rangle$ has been evaluated with $\psi_{\mathbf{r}}=\varphi_{\mathbf{r}}$. This gauge choice is similar to the one made in Sec. III We thus obtain

$$
S=-\frac{i}{2} \rho_{0} \int_{0}^{\beta} d \tau \sum_{\mathbf{r}} \dot{q}_{\mathbf{r}}+\frac{1}{2} \sum_{\tilde{q}}\left(p_{-\tilde{q}}, q_{-\tilde{q}}\right) \mathcal{D}^{-1}(\tilde{q})\left(\begin{array}{c}
p_{\tilde{q}} \\
q_{\tilde{q}}
\end{array}\right)
$$




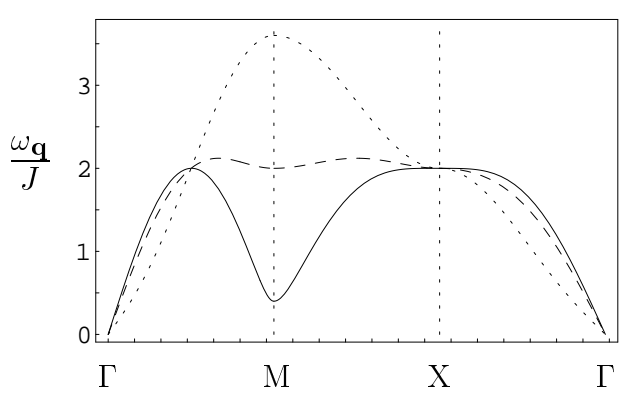

FIG. 12: Collective mode dispersion $\omega_{\mathbf{q}}$ in the strong-coupling limit as obtained from the Heisenberg model [Eq. 95)] for $x=0.1$ (solid line), 0.5 (dashed line) and 0.9 (dotted line). $[\Gamma=(0,0), \mathrm{M}=(\pi, \pi)$ and $\mathrm{X}=(\pi, 0)$.

$$
\mathcal{D}^{-1}(\tilde{q})=\left(\begin{array}{lr}
-4 J \cos \left(2 \theta_{0}\right)\left(1+\gamma_{\mathbf{q}}\right)+4 h_{0} \cos \theta_{0} & \omega_{\nu} \sin \theta_{0} \\
-\omega_{\nu} \sin \theta_{0} & J \sin ^{2} \theta_{0}\left(1-\gamma_{\mathbf{q}}\right)
\end{array}\right)
$$

where $\gamma_{\mathbf{q}}=\left(\cos q_{x}+\cos q_{y}\right) / 2$. Eqs. (92 94) assume the fluctuations of $p$ to be small and are therefore valid only for $h_{0} \neq 0$, i.e. away from half-filling in the attractive model. At zero-temperature, there is AF long-range order (i.e. superconducting order in the attractive model). Collective modes are then obtained from $\operatorname{det} \mathcal{D}^{-1}(\tilde{q})=0$ with the analytic continuation to real frequencies $i \omega_{\nu} \rightarrow \omega_{\mathbf{q}}$. This gives 66

$$
\begin{aligned}
\omega_{\mathbf{q}}^{2} & =2 h_{0}^{2}\left(1-\gamma_{\mathbf{q}}\right)-\left(2 h_{0}^{2}-4 J^{2}\right)\left(1-\gamma_{\mathbf{q}}^{2}\right) \\
& =8 J^{2} x^{2}\left(1-\gamma_{\mathbf{q}}\right)-4 J^{2}\left(2 x^{2}-1\right)\left(1-\gamma_{\mathbf{q}}^{2}\right) .
\end{aligned}
$$

Fig. 12 shows $\omega_{\mathbf{q}}$ for different values of the doping $x$. For $\mathbf{q} \rightarrow 0$, we obtain a spin-wave mode (satisfying $p \simeq 0$ ) with dispersion $\omega_{\mathbf{q}}=c|\mathbf{q}|, c=\sqrt{2} J \sqrt{1-x^{2}}$. This mode corresponds to the Bogoliubov mode obtained in Sec. IID 2 In the vicinity of $\mathbf{Q}=(\pi, \pi)$, we find a mode with the dispersion

$$
\omega_{\mathbf{q}}^{2}=\left(2 h_{0}\right)^{2} \pm \sqrt{2} J\left|1-3 x^{2}\right|^{1 / 2} \mathbf{q}^{2},
$$

where the $+(-)$ sign refers to the case $x<1 / \sqrt{3}(x>1 / \sqrt{3})$. When $x<1 / \sqrt{3}$, this mode corresponds to a local minimum of the energy with a gap $\left|2 h_{0}\right|$. It involves fluctuations along the magnetic field axis which correspond in the attractive model to $\mathbf{q}=\mathbf{Q}$ charge-density fluctuations. At the critical value of the doping $x_{c}=1 / \sqrt{3}$, this local minimum becomes a local maximum. Note that the value $\left|2 h_{0}\right|$ of the gap was also found in Sec. IIIA for a weak magnetic field but for all values of the interaction $U 67$

\section{B. Quantum XY model}

In this section, we show that the attractive Hubbard model in the strong-coupling limit reduces to the quantum $\mathrm{XY}$ model (except in the low-density limit). The effective action of the phase $\Theta_{\mathbf{r}}=-q_{\mathbf{r}}=-\varphi_{\mathbf{r}}+\mathbf{Q} \cdot \mathbf{r}$ of the superconducting order parameter is obtained by integrating out the $p$ field. To quadratic order in $p$, the action reads

$$
\begin{aligned}
S[p, \Theta]= & \int_{0}^{\beta} d \tau\left\{\sum_{\mathbf{r}}\left[\frac{i}{2} \rho_{0} \dot{\Theta}_{\mathbf{r}}-i \sin \theta_{0} p_{\mathbf{r}} \dot{\Theta}_{\mathbf{r}}+2 h_{0} \cos \theta_{0} p_{\mathbf{r}}^{2}\right]-\frac{J}{2} \cos \left(2 \theta_{0}\right) \sum_{\left\langle\mathbf{r}, \mathbf{r}^{\prime}\right\rangle}\left(p_{\mathbf{r}}+p_{\mathbf{r}^{\prime}}\right)^{2}\right. \\
& \left.+\frac{J}{4} \sum_{\left\langle\mathbf{r}, \mathbf{r}^{\prime}\right\rangle}\left[1-\cos \left(\Theta_{\mathbf{r}}-\Theta_{\mathbf{r}^{\prime}}\right)\right]\left[\sin ^{2} \theta_{0}+\sin \left(2 \theta_{0}\right)\left(p_{\mathbf{r}}+p_{\mathbf{r}^{\prime}}\right)+4 \cos ^{2} \theta_{0} p_{\mathbf{r}} p_{\mathbf{r}^{\prime}}-2 \sin ^{2} \theta_{0}\left(p_{\mathbf{r}}^{2}+p_{\mathbf{r}^{\prime}}^{2}\right)\right]\right\} .
\end{aligned}
$$

Since the fluctuations of $p$ are small, we can neglect all the terms but $\sin ^{2} \theta_{0}$ in the coefficient of $1-\cos \left(\Theta_{\mathbf{r}}-\right.$ $\left.\Theta_{\mathbf{r}^{\prime}}\right)$. This approximation breaks down when $\sin \theta_{0} \rightarrow 0$ (i.e. $\theta_{0} \rightarrow \pi$ ), which corresponds to the low-density limit of the attractive Hubbard model. Integrating out the $p$ 
field, we obtain the action of the quantum XY model

$$
\begin{aligned}
S[\Theta]= & \int_{0}^{\beta} d \tau\left\{\sum_{\mathbf{r}}\left[\frac{i}{2} \rho_{0} \dot{\Theta}_{\mathbf{r}}+\frac{\dot{\Theta}_{\mathbf{r}}^{2}}{16 J}\right]\right. \\
& \left.+\frac{J}{4}\left(1-x^{2}\right) \sum_{\left\langle\mathbf{r}, \mathbf{r}^{\prime}\right\rangle}\left[1-\cos \left(\Theta_{\mathbf{r}}-\Theta_{\mathbf{r}^{\prime}}\right)\right]\right\} .
\end{aligned}
$$

Taking the continuum limit, we recover the phase-only action derived in Sec. II for $U \gg 4 t$ [Eqs. (5258)].

\section{Low-density limit: Gross-Pitaevskii equation}

In the strong-coupling limit, fermions form tightly bound pairs which behave as hard-core bosons (the hardcore constraint comes from the Pauli principle which prevents double occupancy of a lattice site). In the lowdensity limit, the hard-core constraint does not matter anymore and we expect to recover the usual action of a Bose superfluid, and in turn the Gross-Pitaevskii equation.

Using Eqs. (4748),

$$
\begin{aligned}
\Omega_{\mathbf{r}}^{ \pm} & =(-1)^{\mathbf{r}}\left[\rho_{\mathbf{r}}\left(2-\rho_{\mathbf{r}}\right)\right]^{1 / 2} e^{\mp i \Theta_{\mathbf{r}}}, \\
\Omega_{\mathbf{r}}^{z} & =\rho_{\mathbf{r}}-1,
\end{aligned}
$$

we deduce from the Heisenberg model [Eq. (89)]

$$
\begin{aligned}
S[\rho, \Theta]= & \int_{0}^{\beta} d \tau \int d^{2} r\left\{\frac{i}{2} \rho_{\mathbf{r}} \dot{\Theta}_{\mathbf{r}}+J \rho_{\mathbf{r}}^{2}-\left(h_{0}+2 J\right) \rho_{\mathbf{r}}\right. \\
& \left.+\frac{J}{4}\left[\frac{\left(\nabla \rho_{\mathbf{r}}\right)^{2}}{4 \rho_{\mathbf{r}}}+\rho_{\mathbf{r}}\left(\nabla \Theta_{\mathbf{r}}\right)^{2}\right]\right\}
\end{aligned}
$$

in the low-density limit $\left(\rho_{\mathbf{r}} \ll 1\right)$ and to second order in gradient. We have taken the continuum limit in real space. Introducing the boson density $\rho_{b \mathbf{r}}=\rho_{\mathbf{r}} / 2$, we recover the action of a Bose superfluid,

$$
\begin{aligned}
S\left[\rho_{b}, \Theta\right]= & \int_{0}^{\beta} d \tau \int d^{2} r\left\{i \rho_{b \mathbf{r}} \dot{\Theta}_{\mathbf{r}}+\frac{g}{2} \rho_{b \mathbf{r}}^{2}-\mu_{b} \rho_{b \mathbf{r}}\right. \\
& \left.+\frac{1}{2 m_{b}}\left[\frac{\left(\nabla \rho_{b \mathbf{r}}\right)^{2}}{4 \rho_{b \mathbf{r}}}+\rho_{b \mathbf{r}}\left(\nabla \Theta_{\mathbf{r}}\right)^{2}\right]\right\} \\
= & \int_{0}^{\beta} d \tau \int d^{2} r\left[\Psi_{\mathbf{r}}^{*}\left(\partial_{\tau}-\mu-\frac{\nabla^{2}}{2 m_{b}}\right) \Psi_{\mathbf{r}}\right. \\
& \left.+\frac{g}{2}\left(\Psi_{\mathbf{r}}^{*} \Psi_{\mathbf{r}}\right)^{2}\right]
\end{aligned}
$$

where $\Psi_{\mathbf{r}}=\sqrt{\rho_{b \mathbf{r}}} e^{i \Theta_{\mathbf{r}}}$.

$$
\begin{aligned}
m_{b} & =\frac{1}{J}, \\
\mu_{b} & =2 h_{0}+4 J, \\
g & =8 J,
\end{aligned}
$$

are the mass, chemical potential and interaction constant of the bosons, respectively. Note that if we neglect the $\left(\nabla \rho_{b \mathbf{r}}\right)^{2}$ term and replace $\rho_{b \mathbf{r}}$ by $\rho_{b 0}$ in the $\left(\nabla \Theta_{\mathbf{r}}\right)^{2}$ term, we recover the action $S[\rho, \Theta]$ (for $x \rightarrow 1$ ) derived in Sec. IIC 2

The classical equations of motion derived from the action (101) yield the Gross-Pitaevskii equation

$$
\left(\partial_{\tau}-\mu_{b}-\frac{\nabla^{2}}{2 m_{b}}\right) \Psi_{\mathbf{r}}+g\left|\Psi_{\mathbf{r}}\right|^{2} \Psi_{\mathbf{r}}=0
$$

for the complex order parameter $\Psi_{\mathbf{r}}=\sqrt{\rho_{b \mathbf{r}}} e^{i \Theta_{\mathbf{r}}}$. As pointed out in Sec. IIC2 $\Psi_{\mathbf{r}}$ equals the superconducting order parameter $\Delta_{\mathbf{r}}=\left|\Delta_{\mathbf{r}}\right| e^{i \Theta_{\mathbf{r}}}$ in the strong-coupling low-density limit of the attractive Hubbard model. Alternatively, the Gross-Pitaevskii equation can be obtained from the semiclassical spin dynamics,

$$
i \dot{\Omega}_{\mathbf{r}}=\frac{J}{2} \sum_{\delta} \boldsymbol{\Omega}_{\mathbf{r}+\boldsymbol{\delta}} \times \boldsymbol{\Omega}_{\mathbf{r}}-2 \mathbf{h}_{0} \times \boldsymbol{\Omega}_{\mathbf{r}}
$$

within a second-order gradient expansion and in the lowdensity limit $\rho_{\mathbf{r}}=\Omega_{\mathbf{r}}^{z}+1 \ll 1$. The sum over $\boldsymbol{\delta}$ in (104) denotes a sum over nearest neighbors.

For continuum models, the time-independent GrossPitaevskii equation has been obtained as the strongcoupling limit of the Bogoliubov-de Gennes equations. 68 It has also been shown that the results obtained in the strong-coupling limit of an RPA calculation about the BCS state can be reproduced from the linearized version of the time-dependent Gross-Pitaevskii equation. 69 In this section, we have directly shown the equivalence, in the low-density limit of the lattice case, between the low-energy effective action of the superfluid order parameter $\Delta_{\mathbf{r}} \equiv \sqrt{\rho_{\mathbf{r}} / 2} e^{i \Theta_{\mathbf{r}}}$ and the action of a Bose superfluid. Our approach is not limited to the low-density limit. Eq. (104), together with (99), holds for any density and can be considered as a generalization of the GrossPitaevskii equation to the lattice case.

\section{CONCLUDING REMARKS}

In this paper, we have studied the $2 \mathrm{D}$ attractive Hubbard model using the mapping onto the half-filled repulsive model in a uniform magnetic field coupled to the fermion spins. Our approach reproduces, in a unique framework, a number of previously known results.

One of our main new results is the derivation of a low-energy effective action $S[\rho, \Theta]$ which is valid for all values of doping $x=1-\rho_{0}$ and interaction strength $U$. $S[\rho, \Theta]$ has been obtained by integrating out amplitude fluctuations $(|\Delta|)$ and therefore does not describe the full dynamics of the superconducting order parameter $\Delta_{\mathbf{r}}=\left|\Delta_{\mathbf{r}}\right| e^{i \Theta_{\mathbf{r}}}$. Nevertheless, it is similar to the action of a Bose superfluid with order parameter $\Psi_{\mathbf{r}}=\sqrt{\rho_{\mathbf{r}} / 2} e^{i \Theta_{\mathbf{r}}}$ where the mass of the "bosons" and their mutual interaction depend on $x$ and $U$. This ensures that a Fermi superfluid, as described by the $2 \mathrm{D}$ attractive Hubbard 
model, will behave similarly to a Bose superfluid and exhibit the same macroscopic quantum phenomena. The effective action $S[\rho, \Theta]$ also describes the smooth crossover between the weak-coupling BCS limit and the Bose limit of preformed (local) pairs.

Another important result obtained by our approach is a complete description of the phase diagram of the $2 \mathrm{D}$ attractive Hubbard model. From the phase-only action $S[\Theta]$, we are able to extract an effective XY model and in turn the BKT phase transition temperature $T_{\mathrm{BKT}}$. We identify a $\mathrm{RC}$ regime of superconducting fluctuations in the temperature range $T_{\mathrm{BKT}} \leq T \leq T_{X}$ and an incoherent-pair regime (with no superconducting shortrange order) for $T_{X} \leq T \leq T_{\text {pair }}$. The values obtained for $T_{\text {pair }}$ and $T_{X}$ are in good agreement with numerical results (when available). Near half-filling, we find that $T_{\mathrm{BKT}}$ is suppressed due to the strong $\mathbf{q}=(\pi, \pi)$ charge fluctuations which enlarge the symmetry of the order pa- rameter to $\mathrm{SO}(3)$.

In the strong-coupling limit, the attractive Hubbard model maps onto the Heisenberg model in a uniform field. The latter reduces to the quantum XY model (except for a weak field, i.e. in the low-density limit of the attractive model). In the low-density limit, we recover the usual action of a Bose superfluid (including the terms proportional to $\left.\left(\boldsymbol{\nabla} \rho_{\mathbf{r}}\right)^{2}\right)$ and in turn the Gross-Pitaevskii equation.

\section{Acknowledgments}

We thank A.M.-S. Tremblay and B. Delamotte for discussions and K. Borejsza for advice regarding the numerical calculations.

\section{APPENDIX A: HF CURRENT-CURRENT CORRELATION FUNCTION}

In this appendix we calculate the HF current-current correlation function

$$
\Pi_{\mu \mu^{\prime}}^{\nu \nu^{\prime}}\left(\tilde{q}, \tilde{q}^{\prime}\right)=\left\langle j_{\mu}^{\nu}(\tilde{q}) j_{\mu^{\prime}}^{\nu^{\prime}}\left(-\tilde{q}^{\prime}\right)\right\rangle_{c}
$$

for $\tilde{q}, \tilde{q}^{\prime}=0$, Q. The calculation is performed at finite temperature in the classical limit: $\lim _{\tilde{q} \rightarrow 0} \equiv \lim _{\mathbf{q} \rightarrow 0} \lim _{\omega_{\nu} \rightarrow 0}$ and $\lim _{\tilde{q} \rightarrow \mathbf{Q}} \equiv \lim _{\mathbf{q} \rightarrow \mathbf{Q}} \lim _{\omega_{\nu} \rightarrow 0} \cdot j_{\mu}^{\nu}(\tilde{q})$ is the Fourier transformed field of

$$
\begin{aligned}
& j_{0 \mathbf{r}}^{\nu}=\phi_{\mathbf{r}}^{\dagger} \sigma^{\nu} \phi_{\mathbf{r}}, \\
& j_{\mu \mathbf{r}}^{\nu}=-i t \phi_{\mathbf{r}}^{\dagger} \sigma^{\nu} \phi_{\mathbf{r}+\hat{\mu}}+\text { c.c. }(\mu=x, y) .
\end{aligned}
$$

We have

$$
\begin{aligned}
& j_{\mu}^{\nu}(\tilde{q})=\frac{1}{\sqrt{\beta N}} \sum_{\tilde{k}} v_{\mu}(\mathbf{k}, \mathbf{q}) \phi_{\tilde{k}}^{\dagger} \sigma^{\nu} \phi_{\tilde{k}+\tilde{q}}, \\
& v_{0}(\mathbf{k}, \mathbf{q})=1, \\
& v_{\mu \neq 0}(\mathbf{k}, \mathbf{q})=-i t\left(e^{i\left(k_{\mu}+q_{\mu}\right)}-e^{-i k_{\mu}}\right) .
\end{aligned}
$$

$\tilde{k}=(\mathbf{k}, i \omega)$ where $\omega=\pi T(2 n+1)$ ( $n$ integer $)$ is a fermionic Matsubara frequency. In (A1), one must have $\tilde{q}=\tilde{q}^{\prime}$ or $\tilde{q}=\tilde{q}^{\prime}+\mathbf{Q}$. The correlation functions of interest are

$$
\begin{aligned}
\Pi_{\mu \mu^{\prime}}^{\nu \nu \prime^{\prime}}(0,0)= & -\frac{1}{\beta N} \sum_{\tilde{k}, \sigma_{1}, \sigma_{2}}\left[v_{\mu}(\mathbf{k}, 0) v_{\mu^{\prime}}(\mathbf{k}, 0) \sigma_{\sigma_{1} \sigma_{2}}^{\nu} \sigma_{\sigma_{2} \sigma_{1}}^{\nu^{\prime}} G_{\sigma_{1}}(\tilde{k}) G_{\sigma_{2}}(\tilde{k})\right. \\
& \left.+v_{\mu}(\mathbf{k}, 0) v_{\mu^{\prime}}(\mathbf{k}+\mathbf{Q}, 0) \sigma_{\sigma_{1} \sigma_{2}}^{\nu} \sigma_{\bar{\sigma}_{2} \bar{\sigma}_{1}}^{\nu^{\prime}} F_{\sigma_{1}}(\tilde{k}) F_{\sigma_{2}}(\tilde{k})\right], \\
\Pi_{\mu \mu^{\prime}}^{\nu \nu^{\prime}}(\mathbf{Q}, \mathbf{Q})= & -\frac{1}{\beta N} \sum_{\tilde{k}, \sigma_{1}, \sigma_{2}}\left[v_{\mu}(\mathbf{k}, \mathbf{Q}) v_{\mu^{\prime}}(\mathbf{k}+\mathbf{Q},-\mathbf{Q}) \sigma_{\sigma_{1} \sigma_{2}}^{\nu} \sigma_{\sigma_{2} \sigma_{1}}^{\nu^{\prime}} G_{\sigma_{1}}(\tilde{k}) G_{\sigma_{2}}(\tilde{k}+\mathbf{Q})\right. \\
& \left.+v_{\mu}(\mathbf{k}, \mathbf{Q}) v_{\mu^{\prime}}(\mathbf{k},-\mathbf{Q}) \sigma_{\sigma_{1} \sigma_{2}}^{\nu} \sigma_{\bar{\sigma}_{2} \bar{\sigma}_{1}}^{\nu^{\prime}} F_{\sigma_{1}}(\tilde{k}) F_{\sigma_{2}}(\tilde{k}+\mathbf{Q})\right], \\
\Pi_{\mu \mu^{\prime}}^{\nu \nu^{\prime}}(0, \mathbf{Q})= & -\frac{1}{\beta N} \sum_{\tilde{k}, \sigma_{1}, \sigma_{2}}\left[v_{\mu}(\mathbf{k}, 0) v_{\mu^{\prime}}(\mathbf{k},-\mathbf{Q}) \sigma_{\sigma_{1} \sigma_{2}}^{\nu} \sigma_{\sigma_{2}}^{\nu^{\prime}} F_{\sigma_{1}} F_{\sigma_{1}}(\tilde{k}) G_{\sigma_{2}}(\tilde{k})\right. \\
& \left.+v_{\mu}(\mathbf{k}, 0) v_{\mu^{\prime}}(\mathbf{k}+\mathbf{Q},-\mathbf{Q}) \sigma_{\sigma_{1} \sigma_{2}}^{\nu} \sigma_{\bar{\sigma}_{2} \sigma_{1}}^{\nu^{\prime}} G_{\sigma_{1}}(\tilde{k}) F_{\sigma_{2}}(\tilde{k})\right],
\end{aligned}
$$


where

$$
\begin{aligned}
v_{\mu}(\mathbf{k}, 0) & =\delta_{\mu, 0}+\delta_{\mu \neq 0} 2 t \sin k_{\mu}, \\
v_{\mu}(\mathbf{k}+\mathbf{Q}, 0) & =\gamma_{\mu} v_{\mu}(\mathbf{k}, 0), \\
v_{\mu}(\mathbf{k}, \mathbf{Q}) & =\delta_{\mu, 0}+\delta_{\mu \neq 0} 2 i t \cos k_{\mu}, \\
v_{\mu}(\mathbf{k}+\mathbf{Q},-\mathbf{Q}) & =\gamma_{\mu} v_{\mu}(\mathbf{k}, \mathbf{Q}),
\end{aligned}
$$

and $\gamma_{\mu}=\delta_{\mu, 0}-\delta_{\mu \neq 0}$. We use the notation $\delta_{\mu \neq 0}=1-\delta_{\mu, 0}$ and $\bar{\sigma}=-\sigma$. The HF propagators $G$ and $F$ are defined in (16). We have

$$
\begin{aligned}
\frac{1}{\beta} \sum_{\omega} G_{\sigma}(\mathbf{k}, i \omega) G_{\sigma^{\prime}}\left(\mathbf{k}^{\prime}, i \omega\right) & =\frac{-1}{2\left(E_{\mathbf{k} \sigma}^{2}-E_{\mathbf{k}^{\prime} \sigma^{\prime}}^{2}\right)}\left[T_{\mathbf{k} \sigma}\left(E_{\mathbf{k} \sigma}+\frac{\epsilon_{\mathbf{k} \sigma} \epsilon_{\mathbf{k}^{\prime} \sigma^{\prime}}}{E_{\mathbf{k} \sigma}}\right)-T_{\mathbf{k}^{\prime} \sigma^{\prime}}\left(E_{\mathbf{k}^{\prime} \sigma^{\prime}}+\frac{\epsilon_{\mathbf{k} \sigma} \epsilon_{\mathbf{k}^{\prime} \sigma^{\prime}}}{E_{\mathbf{k}^{\prime} \sigma^{\prime}}}\right)\right], \\
\frac{1}{\beta} \sum_{\omega} G_{\sigma}(\mathbf{k}, i \omega) G_{\sigma^{\prime}}\left(\mathbf{k}^{\prime}+\mathbf{Q}, i \omega\right) & =\frac{-1}{2\left(E_{\mathbf{k} \sigma}^{2}-E_{\mathbf{k}^{\prime} \sigma^{\prime}}^{2}\right)}\left[T_{\mathbf{k} \sigma}\left(E_{\mathbf{k} \sigma}-\frac{\epsilon_{\mathbf{k} \sigma} \epsilon_{\mathbf{k}^{\prime} \bar{\sigma}^{\prime}}}{E_{\mathbf{k} \sigma}}\right)-T_{\mathbf{k}^{\prime} \bar{\sigma}^{\prime}}\left(E_{\mathbf{k}^{\prime} \bar{\sigma}^{\prime}}-\frac{\epsilon_{\mathbf{k} \sigma} \epsilon_{\mathbf{k}^{\prime} \bar{\sigma}^{\prime}}}{E_{\mathbf{k}^{\prime} \sigma^{\prime}}}\right)\right], \\
\frac{1}{\beta} \sum_{\omega} F_{\sigma}(\mathbf{k}, i \omega) F_{\sigma^{\prime}}\left(\mathbf{k}^{\prime}, i \omega\right) & =\frac{-\Delta_{0}^{\mathrm{HS}}{ }^{2}}{2\left(E_{\mathbf{k} \sigma}^{2}-E_{\mathbf{k}^{\prime} \sigma^{\prime}}^{2}\right)}\left(\frac{T_{\mathbf{k} \sigma}}{E_{\mathbf{k} \sigma}}-\frac{T_{\mathbf{k}^{\prime} \sigma^{\prime}}}{E_{\mathbf{k}^{\prime} \sigma^{\prime}}}\right), \\
\frac{1}{\beta} \sum_{\omega} F_{\sigma}(\mathbf{k}, i \omega) G_{\sigma^{\prime}}\left(\mathbf{k}^{\prime}, i \omega\right) & =\frac{\Delta_{0}^{\mathrm{HS}} \epsilon_{\mathbf{k}^{\prime} \sigma^{\prime}}}{2\left(E_{\mathbf{k} \sigma}^{2}-E_{\mathbf{k}^{\prime} \sigma^{\prime}}^{2}\right)}\left(\frac{T_{\mathbf{k} \sigma}}{E_{\mathbf{k} \sigma}}-\frac{T_{\mathbf{k}^{\prime} \sigma^{\prime}}}{E_{\mathbf{k}^{\prime} \sigma^{\prime}}}\right) .
\end{aligned}
$$

where $T_{\mathbf{k} \sigma}=\tanh \left(\beta E_{\mathbf{k} \sigma} / 2\right)$. Performing the sum over $\sigma_{1}, \sigma_{2}$ in (A4), we find that the only non-vanishing correlation functions are [using $\left.\Pi_{\mu \mu^{\prime}}^{\nu \nu^{\prime}}\left(\tilde{q}, \tilde{q}^{\prime}\right)=\Pi_{\mu^{\prime} \mu}^{\nu^{\prime} \nu}\left(\tilde{q}^{\prime}, \tilde{q}\right)\right]$

$$
\begin{aligned}
& \Pi_{\mu \mu}^{z z}(0,0)=\frac{1}{2} \int_{\mathbf{k}} v_{\mu}^{2}(\mathbf{k}, 0)\left[\frac{T_{\mathbf{k} \uparrow}}{E_{\mathbf{k} \uparrow}^{3}} \Delta_{0}^{\mathrm{HS}^{2}}\left(1+\gamma_{\mu}\right)+\frac{U_{\mathbf{k} \uparrow}}{E_{\mathbf{k} \uparrow}^{2}}\left(E_{\mathbf{k} \uparrow}^{2}+\epsilon_{\mathbf{k} \uparrow}^{2}-\gamma_{\mu} \Delta_{0}^{\mathrm{HS}}{ }^{2}\right)\right], \\
& \Pi_{\mu \mu}^{x x}(0,0)=\int_{\mathbf{k}} \frac{v_{\mu}^{2}(\mathbf{k}, 0)}{E_{\mathbf{k} \uparrow}^{2}-E_{\mathbf{k} \downarrow}^{2}}\left[\frac{T_{\mathbf{k} \uparrow}}{E_{\mathbf{k} \uparrow}}\left(E_{\mathbf{k} \uparrow}^{2}+\epsilon_{\mathbf{k} \uparrow} \epsilon_{\mathbf{k} \downarrow}+\gamma_{\mu} \Delta_{0}^{\mathrm{HS}}{ }^{2}\right)-\frac{T_{\mathbf{k} \downarrow}}{E_{\mathbf{k} \downarrow}}\left(E_{\mathbf{k} \downarrow}^{2}+\epsilon_{\mathbf{k} \uparrow} \epsilon_{\mathbf{k} \downarrow}+\gamma_{\mu} \Delta_{0}^{\mathrm{HS}}{ }^{2}\right)\right], \\
& \Pi_{\mu \mu}^{y y}(0,0)=\int_{\mathbf{k}} \frac{v_{\mu}^{2}(\mathbf{k}, 0)}{E_{\mathbf{k} \uparrow}^{2}-E_{\mathbf{k} \downarrow}^{2}}\left[\frac{T_{\mathbf{k} \uparrow}}{E_{\mathbf{k} \uparrow}}\left(E_{\mathbf{k} \uparrow}^{2}+\epsilon_{\mathbf{k} \uparrow} \epsilon_{\mathbf{k} \downarrow}-\gamma_{\mu} \Delta_{0}^{\mathrm{HS}}{ }^{2}\right)-\frac{T_{\mathbf{k} \downarrow}}{E_{\mathbf{k} \downarrow}}\left(E_{\mathbf{k} \downarrow}^{2}+\epsilon_{\mathbf{k} \uparrow} \epsilon_{\mathbf{k} \downarrow}-\gamma_{\mu} \Delta_{0}^{\mathrm{HS}}{ }^{2}\right)\right], \\
& \Pi_{\mu \mu^{\prime}}^{z z}(\mathbf{Q}, \mathbf{Q})=\bar{\delta}_{\mu, \mu^{\prime}} \int_{\mathbf{k}} \frac{v_{\mu}(\mathbf{k}, \mathbf{Q}) v_{\mu^{\prime}}(\mathbf{k}, \mathbf{Q})}{E_{\mathbf{k} \uparrow}^{2}-E_{\mathbf{k} \downarrow}^{2}}\left[\frac{T_{\mathbf{k} \uparrow}}{E_{\mathbf{k} \uparrow}}\left(\gamma_{\mu}\left(E_{\mathbf{k} \uparrow}^{2}-\epsilon_{\mathbf{k} \uparrow} \epsilon_{\mathbf{k} \downarrow}\right)-\Delta_{0}^{\mathrm{HS}^{2}}\right)-\frac{T_{\mathbf{k} \downarrow}}{E_{\mathbf{k} \downarrow}}\left(\gamma_{\mu}\left(E_{\mathbf{k} \downarrow}^{2}-\epsilon_{\mathbf{k} \uparrow} \epsilon_{\mathbf{k} \downarrow}\right)-\Delta_{0}^{\mathrm{HS}^{2}}\right)\right], \\
& \Pi_{\mu \mu^{\prime}}^{x x}(\mathbf{Q}, \mathbf{Q})=-\bar{\delta}_{\mu, \mu^{\prime}} \gamma_{\mu} \frac{1}{2} \int_{\mathbf{k}} v_{\mu}(\mathbf{k}, \mathbf{Q}) v_{\mu^{\prime}}(\mathbf{k}, \mathbf{Q})\left[\frac{T_{\mathbf{k} \uparrow}}{E_{\mathbf{k} \uparrow}}\left(-2+\frac{\Delta_{0}^{\mathrm{HS}^{2}}}{E_{\mathbf{k} \uparrow}^{2}}\left(1+\gamma_{\mu}\right)\right)-U_{\mathbf{k} \uparrow} \frac{\Delta_{0}^{\mathrm{HS}^{2}}}{E_{\mathbf{k} \uparrow}^{2}}\left(1+\gamma_{\mu}\right)\right], \\
& \Pi_{\mu \mu^{\prime}}^{y y}(\mathbf{Q}, \mathbf{Q})=-\bar{\delta}_{\mu, \mu^{\prime}} \gamma_{\mu} \frac{1}{2} \int_{\mathbf{k}} v_{\mu}(\mathbf{k}, \mathbf{Q}) v_{\mu^{\prime}}(\mathbf{k}, \mathbf{Q})\left[\frac{T_{\mathbf{k} \uparrow}}{E_{\mathbf{k} \uparrow}}\left(-2+\frac{\Delta_{0}^{\mathrm{HS}}{ }^{2}}{E_{\mathbf{k} \uparrow}^{2}}\left(1-\gamma_{\mu}\right)\right)-U_{\mathbf{k} \uparrow} \frac{\Delta_{0}^{\mathrm{HS}^{2}}}{E_{\mathbf{k} \uparrow}^{2}}\left(1-\gamma_{\mu}\right)\right], \\
& \Pi_{0, \mu \neq 0}^{x y}(\mathbf{Q}, \mathbf{Q})=-\frac{1}{2} \int_{\mathbf{k}} \epsilon_{\mathbf{k}}\left(T_{\mathbf{k} \uparrow} \frac{\epsilon_{\mathbf{k} \uparrow}^{2}}{E_{\mathbf{k} \uparrow}^{3}}+U_{\mathbf{k} \uparrow} \frac{\Delta_{0}^{\mathrm{HS}^{2}}}{E_{\mathbf{k} \uparrow}^{2}}\right), \\
& \Pi_{\mu \neq 0,0}^{x y}(\mathbf{Q}, \mathbf{Q})=\frac{h_{0}}{U}, \\
& \Pi_{00}^{z x}(0, \mathbf{Q})=\Delta_{0}^{\mathrm{HS}} \int_{\mathbf{k}} \epsilon_{\mathbf{k} \uparrow}\left(\frac{T_{\mathbf{k} \uparrow}}{E_{\mathbf{k} \uparrow}^{3}}-\frac{U_{\mathbf{k} \uparrow}}{E_{\mathbf{k} \uparrow}^{2}}\right), \\
& \Pi_{0, \mu \neq 0}^{z y}(0, \mathbf{Q})=-\frac{\Delta_{0}^{\mathrm{HS}}}{2} \int_{\mathbf{k}} \epsilon_{\mathbf{k}} \epsilon_{\mathbf{k} \uparrow}\left(\frac{T_{\mathbf{k} \uparrow}}{E_{\mathbf{k} \uparrow}^{3}}-\frac{U_{\mathbf{k} \uparrow}}{E_{\mathbf{k} \uparrow}^{2}}\right), \\
& \Pi_{00}^{x z}(0, \mathbf{Q})=2 \Delta_{0}^{\mathrm{HS}} h \int_{\mathbf{k}} \frac{1}{E_{\mathbf{k} \uparrow}^{2}-E_{\mathbf{k} \downarrow}^{2}}\left(\frac{T_{\mathbf{k} \uparrow}}{E_{\mathbf{k} \uparrow}}-\frac{T_{\mathbf{k} \downarrow}}{E_{\mathbf{k} \downarrow}}\right), \\
& \Pi_{0, \mu \neq 0}^{y z}(0, \mathbf{Q})=-\Delta_{0}^{\mathrm{HS}} \int_{\mathbf{k}} \frac{\epsilon_{\mathbf{k}}^{2}}{E_{\mathbf{k} \uparrow}^{2}-E_{\mathbf{k} \downarrow}^{2}}\left(\frac{T_{\mathbf{k} \uparrow}}{E_{\mathbf{k} \uparrow}}-\frac{T_{\mathbf{k} \downarrow}}{E_{\mathbf{k} \downarrow}}\right),
\end{aligned}
$$


where $U_{\mathbf{k} \sigma}=\beta / 2 \cosh ^{2}\left(\frac{\beta E_{\mathbf{k} \sigma}}{2}\right)$ and $\bar{\delta}_{\mu, \mu^{\prime}}=\delta_{\mu, 0} \delta_{\mu^{\prime}, 0}+\delta_{\mu \neq 0} \delta_{\mu^{\prime} \neq 0}$. In order to obtain Eqs. (A7), we have used the gap equations (22,23) and the symmetry relations $\epsilon_{\mathbf{k} \sigma}=-\epsilon_{\mathbf{k}+\mathbf{Q} \bar{\sigma}}, E_{\mathbf{k} \sigma}=E_{\mathbf{k}+\mathbf{Q} \bar{\sigma}}, v_{\mu \neq 0}(\mathbf{k}, 0)=-v_{\mu \neq 0}(-\mathbf{k}, 0)=$ $-v_{\mu \neq 0}(\mathbf{k}+\mathbf{Q}, 0)$, etc.

1. Strong-coupling limit $U \gg 4 t(T=0)$

Expanding Eqs. A7 to leading order in $1 / U$, we obtain $(T=0)$

$$
\begin{aligned}
\Pi_{00}^{z z}(0,0) & =\frac{2}{U} \sin ^{2} \theta_{0}, \\
\Pi_{00}^{x x}(\mathbf{Q}, \mathbf{Q}) & =\frac{2}{U} \cos ^{2} \theta_{0}, \\
\sum_{\mu, \mu^{\prime}=x, y} \Pi_{\mu \mu^{\prime}}^{y y}(\mathbf{Q}, \mathbf{Q}) & =2 J \cos ^{2} \theta_{0}, \\
\Pi_{0 x}^{x y}(\mathbf{Q}, \mathbf{Q}) & =\frac{8 t^{2}}{U^{2}} \cos \theta_{0}\left(2-3 \cos ^{2} \theta_{0}\right), \\
\Pi_{00}^{z x}(0, \mathbf{Q}) & =-\frac{1}{U} \sin \left(2 \theta_{0}\right), \\
\Pi_{0 x}^{z y}(0, \mathbf{Q}) & =-\frac{8 t^{2}}{U^{2}} \sin \theta_{0}\left(1-3 \cos ^{2} \theta_{0}\right) .
\end{aligned}
$$

Here we consider only the correlation functions that are useful for the derivation of the effective action $S[\rho, \Delta]$ and the calculation of the velocity $c$ of the phase collective mode.

\section{Correlation functions for $h_{0}=0$ and $\boldsymbol{\Omega}_{\mathbf{r}}^{\mathrm{cl}}=(-1)^{\mathbf{r}} \hat{\mathbf{z}}(T=0)$}

In Sec. III we need the HF current-current correlation function at half filling and for a magnetization parallel to the $z$ axis. They can be deduced from Eqs. (A7) with $h_{0}=h=0$ and by making the rotation in spin space $\hat{\mathbf{x}} \rightarrow \hat{\mathbf{z}}$, $\hat{\mathbf{z}} \rightarrow \hat{\mathbf{y}}$ and $\hat{\mathbf{y}} \rightarrow \hat{\mathbf{x}}$. The only non-vanishing correlation functions are then (the notations are those of Sec. III)

$$
\begin{aligned}
\Pi_{00}^{x x}(0,0) & =\Pi_{00}^{y y}(0,0)=m_{0}^{2} \int_{\mathbf{k}} \frac{1}{E_{\mathbf{k}}^{3}}, \\
\Pi_{x x}^{z z}(0,0) & =\Pi_{y y}^{z z}(0,0)=4 t^{2} m_{0}^{2} \int_{\mathbf{k}} \frac{\sin ^{2} k_{x}}{E_{\mathbf{k}}^{3}}, \\
\Pi_{\mu \mu}^{x x}(\mathbf{Q}, \mathbf{Q}) & =\Pi_{\mu \mu}^{y y}(\mathbf{Q}, \mathbf{Q})=-\int_{\mathbf{k}} \frac{\epsilon_{\mathbf{k}}^{2}}{2 E_{\mathbf{k}}^{3}}\left(\delta_{\mu, 0} E_{\mathbf{k}}^{2}-\delta_{\mu \neq 0} \epsilon_{\mathbf{k}}^{2}\right), \\
\Pi_{\mu \mu}^{z z}(\mathbf{Q}, \mathbf{Q}) & =\int_{\mathbf{k}} \frac{\epsilon_{\mathbf{k}}^{2}}{2 E_{\mathbf{k}}^{3}}\left(\delta_{\mu \neq 0} E_{\mathbf{k}}^{2}-\delta_{\mu, 0} \epsilon_{\mathbf{k}}^{2}\right), \\
\Pi_{0, \mu \neq 0}^{y x}(0, \mathbf{Q}) & =-\frac{m_{0}}{2} \int_{\mathbf{k}} \frac{\epsilon_{\mathbf{k}}^{2}}{E_{\mathbf{k}}^{3}}, \\
\Pi_{0, \mu \neq 0}^{x y}(0, \mathbf{Q}) & =\frac{m_{0}}{2} \int_{\mathbf{k}} \frac{\epsilon_{\mathbf{k}}^{2}}{E_{\mathbf{k}}^{3}},
\end{aligned}
$$

where $E_{\mathbf{k}}=\sqrt{\epsilon_{\mathbf{k}}^{2}+m_{0}^{2}}$.

\section{APPENDIX B: EFFECTIVE ACTION $S[m, \boldsymbol{\Omega}]$}

In this appendix, we derive the effective action $S[m, \boldsymbol{\Omega}]=S[p, q, m]$ [Eq. (34)] of spin fluctuations in the repulsive Hubbard model. 
It is convenient to express the rotation matrix $R_{\mathbf{r}}$ as $R_{\mathbf{r}}=M\left(\boldsymbol{\Omega}_{\mathbf{r}}\right) M^{\dagger}\left(\boldsymbol{\Omega}_{\mathbf{r}}^{\text {cl }}\right)$ where $M\left(\boldsymbol{\Omega}_{\mathbf{r}}\right)$ is defined by $M\left(\boldsymbol{\Omega}_{\mathbf{r}}\right) \sigma^{z} M^{\dagger}\left(\boldsymbol{\Omega}_{\mathbf{r}}\right)=\boldsymbol{\sigma} \cdot \boldsymbol{\Omega}_{\mathbf{r}}$

$$
M\left(\boldsymbol{\Omega}_{\mathbf{r}}\right)=\left(\begin{array}{lr}
\cos \left(\frac{\theta_{\mathbf{r}}}{2}\right) e^{-\frac{i}{2} \varphi_{\mathbf{r}}} & -\sin \left(\frac{\theta_{\mathbf{r}}}{2}\right) e^{-\frac{i}{2} \varphi_{\mathbf{r}}} \\
\sin \left(\frac{\theta_{\mathbf{r}}}{2}\right) e^{\frac{i}{2} \varphi_{\mathbf{r}}} & \cos \left(\frac{\theta_{\mathbf{r}}}{2}\right) e^{\frac{i}{2} \varphi_{\mathbf{r}}}
\end{array}\right)
$$

We then obtain

$$
\begin{aligned}
A_{0 \mathbf{r}}^{x} & =(-1)^{\mathbf{r}}\left[\left(\frac{i}{2} \dot{q}_{\mathbf{r}}+h_{0}\right) \sin \left(2 p_{\mathbf{r}}\right)-\sin \theta_{0} i \delta m_{\mathbf{r}}^{\mathrm{HS}}\right], \\
A_{0 \mathbf{r}}^{y} & =-i(-1)^{\mathbf{r}} \dot{p}_{\mathbf{r}}, \\
A_{0 \mathbf{r}}^{z} & =-\left(\frac{i}{2} \dot{q}_{\mathbf{r}}+h_{0}\right) \cos \left(2 p_{\mathbf{r}}\right)+h_{0}-\cos \theta_{0} i \delta m_{\mathbf{r}}^{\mathrm{HS}}, \\
A_{\mathbf{r}, \mathbf{r}^{\prime}}^{0} & =\cos \left(\frac{q_{\mathbf{r}}-q_{\mathbf{r}^{\prime}}}{2}\right) \cos \left(p_{\mathbf{r}}+p_{\mathbf{r}^{\prime}}\right)-1, \\
A_{\mathbf{r}, \mathbf{r}^{\prime}}^{x} & =-i(-1)^{\mathbf{r}} \sin \left(\frac{q_{\mathbf{r}}-q_{\mathbf{r}^{\prime}}}{2}\right) \sin \left(p_{\mathbf{r}}-p_{\mathbf{r}^{\prime}}\right), \\
A_{\mathbf{r}, \mathbf{r}^{\prime}}^{y} & =i(-1)^{\mathbf{r}} \cos \left(\frac{q_{\mathbf{r}}-q_{\mathbf{r}^{\prime}}}{2}\right) \sin \left(p_{\mathbf{r}}+p_{\mathbf{r}^{\prime}}\right), \\
A_{\mathbf{r}, \mathbf{r}^{\prime}}^{z} & =i \sin \left(\frac{q_{\mathbf{r}}-q_{\mathbf{r}^{\prime}}}{2}\right) \cos \left(p_{\mathbf{r}}-p_{\mathbf{r}^{\prime}}\right) .
\end{aligned}
$$

Eqs. (B2) can be rewritten as

$$
\begin{aligned}
A_{0 \mathbf{r}}^{x} & =(-1)^{\mathbf{r}}\left[\frac{i}{2}\left(p_{\mathbf{r}} \dot{q}_{\mathbf{r}}-\dot{p}_{\mathbf{r}} q_{\mathbf{r}}\right)+2 h_{0} p_{\mathbf{r}}-\sin \theta_{0} i \delta m_{\mathbf{r}}^{\mathrm{HS}}\right] \\
A_{0 \mathbf{r}}^{y} & =-i(-1)^{\mathbf{r}} \dot{p}_{\mathbf{r}} \\
A_{0 \mathbf{r}}^{z} & =-\frac{i}{2} \dot{q}_{\mathbf{r}}+2 h_{0} p_{\mathbf{r}}^{2}-\cos \theta_{0} i \delta m_{\mathbf{r}}^{\mathrm{HS}} \\
A_{\mathbf{r}, \mathbf{r}^{\prime}}^{0} & =-\frac{\left(p_{\mathbf{r}}+p_{\mathbf{r}^{\prime}}\right)^{2}}{2}-\frac{\left(q_{\mathbf{r}}-q_{\mathbf{r}^{\prime}}\right)^{2}}{8} \\
A_{\mathbf{r}, \mathbf{r}^{\prime}}^{x} & =-\frac{i}{2}(-1)^{\mathbf{r}}\left(q_{\mathbf{r}}-q_{\mathbf{r}^{\prime}}\right)\left(p_{\mathbf{r}}-p_{\mathbf{r}^{\prime}}\right) \\
A_{\mathbf{r}, \mathbf{r}^{\prime}}^{y} & =i(-1)^{\mathbf{r}}\left(p_{\mathbf{r}}+p_{\mathbf{r}^{\prime}}\right) \\
A_{\mathbf{r}, \mathbf{r}^{\prime}}^{z} & =\frac{i}{2}\left(q_{\mathbf{r}}-q_{\mathbf{r}^{\prime}}\right)
\end{aligned}
$$

to quadratic order in $p_{\mathbf{r}}, \delta m_{\mathbf{r}}^{\mathrm{HS}}, \dot{p}_{\mathbf{r}}, \dot{q}_{\mathbf{r}}$ and $q_{\mathbf{r}}-q_{\mathbf{r}^{\prime}}$.

Keeping terms up to second-order in $p_{\mathbf{r}}, \delta m_{\mathbf{r}}^{\mathrm{HS}}, \delta m_{\mathbf{r}}, \dot{p}_{\mathbf{r}}, \dot{q}_{\mathbf{r}}$ and $q_{\mathbf{r}}-q_{\mathbf{r}^{\prime}}\left(\mathbf{r}, \mathbf{r}^{\prime}\right.$ nearest neighbors), the effective action is given by first- and second-order cumulants of $S_{1}$ and $S_{2}$ with respect to the HF action:

$$
S\left[p, q, m^{\mathrm{HS}}, m\right]=\left\langle S_{1}+S_{2}\right\rangle-\frac{1}{2}\left\langle\left(S_{1}+S_{2}\right)^{2}\right\rangle_{c}+\int_{0}^{\beta} d \tau \sum_{\mathbf{r}}\left(-\frac{U}{4} \delta m_{\mathbf{r}}^{2}+i \delta m_{\mathbf{r}}^{\mathrm{HS}} \delta m_{\mathbf{r}}-\frac{2}{U} m_{0}^{\mathrm{HS}} \delta m_{\mathbf{r}}^{\mathrm{HS}}\right) .
$$

Using

$$
\begin{aligned}
\left\langle\phi_{\mathbf{r}}^{\dagger} \sigma^{0} \phi_{\mathbf{r}}\right\rangle & =1, \\
\left\langle\phi_{\mathbf{r}}^{\dagger} \sigma^{x} \phi_{\mathbf{r}}\right\rangle & =(-1)^{\mathbf{r}} 2 \Delta_{0}, \\
\left\langle\phi_{\mathbf{r}}^{\dagger} \sigma^{y} \phi_{\mathbf{r}}\right\rangle & =0 \\
\left\langle\phi_{\mathbf{r}}^{\dagger} \sigma^{z} \phi_{\mathbf{r}}\right\rangle & =-x,
\end{aligned}
$$

where the averages are taken with the HF action, we obtain

$$
\left\langle S_{1}\right\rangle=\int_{0}^{\beta} d \tau \sum_{\mathbf{r}}\left[i \Delta_{0}\left(p_{\mathbf{r}} \dot{q}_{\mathbf{r}}-\dot{p}_{\mathbf{r}} q_{\mathbf{r}}\right)+4 \Delta_{0} h_{0} p_{\mathbf{r}}-2 \Delta_{0} \sin \theta_{0} i \delta m_{\mathbf{r}}^{\mathrm{HS}}+\frac{i}{2} x \dot{q}_{\mathbf{r}}-2 x h_{0} p_{\mathbf{r}}^{2}+x \cos \theta_{0} i \delta m_{\mathbf{r}}^{\mathrm{HS}}\right] .
$$


Similarly, from ( $\mathbf{r}, \mathbf{r}^{\prime}$ are nearest neighbors)

$$
\begin{aligned}
\left\langle\phi_{\mathbf{r}}^{\dagger} \sigma^{0} \phi_{\mathbf{r}^{\prime}}\right\rangle & =\frac{\langle-K\rangle}{4 t}=\frac{1}{4 t} \int_{\mathbf{k}} \frac{\epsilon_{\mathbf{k}} \epsilon_{\mathbf{k} \uparrow}}{E_{\mathbf{k} \uparrow}} \tanh \frac{\beta E_{\mathbf{k} \uparrow}}{2}, \\
\left\langle\phi_{\mathbf{r}}^{\dagger} \sigma^{x} \phi_{\mathbf{r}^{\prime}}\right\rangle & =0 \\
\left\langle\phi_{\mathbf{r}}^{\dagger} \sigma^{y} \phi_{\mathbf{r}^{\prime}}\right\rangle & =-i(-1)^{\mathbf{r}} \frac{\Delta_{0} h_{0}}{2 t} \\
\left\langle\phi_{\mathbf{r}}^{\dagger} \sigma^{z} \phi_{\mathbf{r}^{\prime}}\right\rangle & =0
\end{aligned}
$$

we deduce

$$
\left\langle S_{2}\right\rangle=\frac{\langle-K\rangle}{4} \int_{0}^{\beta} d \tau \sum_{\left\langle\mathbf{r}, \mathbf{r}^{\prime}\right\rangle}\left[\left(p_{\mathbf{r}}+p_{\mathbf{r}^{\prime}}\right)^{2}+\frac{\left(q_{\mathbf{r}}-q_{\mathbf{r}^{\prime}}\right)^{2}}{4}\right]-4 \Delta_{0} h_{0} \int_{0}^{\beta} d \tau \sum_{\mathbf{r}} p_{\mathbf{r}} .
$$

We have introduced the mean value $\langle K\rangle$ of the kinetic energy per site in the HF state.

When calculating the second-order cumulant, it is sufficient to consider $A_{0 \mathbf{r}}$ and $A_{\mathbf{r}, \mathbf{r}^{\prime}}$ to linear order in $p_{\mathbf{r}}, \delta m_{\mathbf{r}}^{\mathrm{HS}}$, $\dot{p}_{\mathbf{r}}, \dot{q}_{\mathbf{r}}$, and $q_{\mathbf{r}}-q_{\mathbf{r}^{\prime}}$ :

$$
\begin{aligned}
A_{0 \mathbf{r}}^{x} & =(-1)^{\mathbf{r}}\left(2 h_{0} p_{\mathbf{r}}-\sin \theta_{0} i \delta m_{\mathbf{r}}^{\mathrm{HS}}\right), \\
A_{0 \mathbf{r}}^{y} & =-i(-1)^{\mathbf{r}} \dot{p}_{\mathbf{r}}, \\
A_{0 \mathbf{r}}^{z} & =-\frac{i}{2} \dot{q}_{\mathbf{r}}-\cos \theta_{0} i \delta m_{\mathbf{r}}^{\mathrm{HS}}, \\
A_{\mathbf{r}, \mathbf{r}^{\prime}}^{0} & =0 \\
A_{\mathbf{r}, \mathbf{r}^{\prime}}^{x} & =0 \\
A_{\mathbf{r}, \mathbf{r}^{\prime}}^{y} & =i(-1)^{\mathbf{r}}\left(p_{\mathbf{r}}+p_{\mathbf{r}^{\prime}}\right), \\
A_{\mathbf{r}, \mathbf{r}^{\prime}}^{z} & =\frac{i}{2}\left(q_{\mathbf{r}}-q_{\mathbf{r}^{\prime}}\right) .
\end{aligned}
$$

In order to evaluate $\left\langle\left(S_{1}+S_{2}\right)^{2}\right\rangle_{c}$, we write the action $S_{1}+S_{2}$ as

$$
\begin{aligned}
S_{1}+S_{2} & =\int_{0}^{\beta} d \tau \sum_{\substack{\mu=0, x, y \\
\nu=x, y, z}} A_{\mu \mathbf{r}}^{\nu(\mathrm{tot})} j_{\mu \mathbf{r}}^{\nu}, \\
A_{\mu \mathrm{r}}^{\nu(\mathrm{tot})} & =\delta_{\mu, 0} A_{0 \mathbf{r}}^{\nu}-i\left(1-\delta_{\mu, 0}\right) A_{\mu \mathbf{r}}^{\nu},
\end{aligned}
$$

where $A_{\mu \mathbf{r}}^{\nu}=A_{\mathbf{r}, \mathbf{r}+\hat{\mu}}^{\nu}$ for $\mu=x, y$. The spin-density current $j_{\mu \mathbf{r}}^{\nu}$ is defined by (A2). We then have

$$
\left\langle\left(S_{1}+S_{2}\right)^{2}\right\rangle_{c}=\int_{0}^{\beta} d \tau d \tau^{\prime} \sum_{\substack{r, r^{\prime} \\ \mu, \mu^{\prime}=0, x, y \\ \nu, \nu^{\prime}=x, y, z}} A_{\mu \mathbf{r}}^{\nu(\text { tot })}(\tau) \Pi_{\mu \mu^{\prime}}^{\nu \nu^{\prime}}\left(\mathbf{r}, \tau ; \mathbf{r}^{\prime}, \tau^{\prime}\right) A_{\mu^{\prime} \mathbf{r}^{\prime}}^{\nu^{\prime}(\text { tot })}\left(\tau^{\prime}\right),
$$

where

$$
\Pi_{\mu \mu^{\prime}}^{\nu \nu^{\prime}}\left(\mathbf{r}, \tau ; \mathbf{r}^{\prime}, \tau^{\prime}\right)=\left\langle j_{\mu \mathbf{r}}^{\nu}(\tau) j_{\mu^{\prime} \mathbf{r}^{\prime}}^{\nu^{\prime}}\left(\tau^{\prime}\right)\right\rangle_{c}
$$

is the HF current-current correlation function. $\Pi_{\mu \mu^{\prime}}^{\nu \nu^{\prime}}$ is calculated in Appendix $\mathbb{A}$

In order to calculate the second-order cumulant, we write the actions $S_{1}$ and $S_{2}$ [Eqs. (32)] as

$$
\begin{aligned}
S_{1} & =\int_{0}^{\beta} d \tau \sum_{\substack{\mathbf{r} \\
\nu=x, y, z}} A_{0 \mathbf{r}}^{\nu} j_{0 \mathbf{r}}^{\nu}, \\
S_{2} & =-i \int_{0}^{\beta} d \tau \sum_{\substack{\mu=\mathbf{r}, y \\
\nu=x, y, z}} A_{\mu \mathbf{r}}^{\nu} j_{\mu \mathbf{r}}^{\nu},
\end{aligned}
$$


where $A_{\mu \mathbf{r}}^{\nu}=A_{\mathbf{r}, \mathbf{r}+\hat{\boldsymbol{\mu}}}^{\nu}$ for $\mu=x, y . A_{0 \mathbf{r}}^{\nu}$ and $A_{\mu \mathbf{r}}^{\nu}$ are given by Eqs. (B9). The spin-density current $j_{\mu \mathbf{r}}^{\nu}$ is defined in (A2). In Fourier space, we obtain

$$
\begin{aligned}
A_{0}^{\nu}(\tilde{q}) & =z_{0}(\tilde{q})\left[\delta_{\nu, y} p_{\tilde{q}+\mathbf{Q}}+\delta_{\nu, z} \frac{q \tilde{q}}{2}\right]+\delta_{\nu, x}\left[2 h_{0} p_{\tilde{q}+\mathbf{Q}}-\sin \theta_{0} i \delta m_{\tilde{q}+\mathbf{Q}}^{\mathrm{HS}}\right]-\delta_{\nu, z} \cos \theta_{0} i \delta m_{\tilde{q}}^{\mathrm{HS}}, \\
-i A_{\mu}^{\nu}(\tilde{q}) & =z_{\mu}(\tilde{q})\left[\delta_{\nu, y} p_{\tilde{q}+\mathbf{Q}}+\delta_{\nu, z} \frac{q \tilde{q}}{2}\right] \quad(\mu=x, y),
\end{aligned}
$$

where

$$
\begin{aligned}
z_{0}(\tilde{q}) & =-\omega_{\nu}, \\
z_{\mu=x, y}(\tilde{q}) & =1-e^{i q_{\mu}} .
\end{aligned}
$$

We deduce

$$
\begin{aligned}
\left\langle\left(S_{1}+S_{2}\right)^{2}\right\rangle_{c}= & \sum_{\tilde{q}, \tilde{q}^{\prime}}\left\{\sum _ { \mu , \mu ^ { \prime } = 0 , x , y } \left[z_{\mu}(-\tilde{q}-\mathbf{Q}) z_{\mu^{\prime}}\left(\tilde{q}^{\prime}+\mathbf{Q}\right) p_{-\tilde{q}} p_{\tilde{q}^{\prime}} \Pi_{\mu \mu^{\prime}}^{y y}\left(\tilde{q}+\mathbf{Q}, \tilde{q}^{\prime}+\mathbf{Q}\right)+\frac{1}{4} z_{\mu}(-\tilde{q}) z_{\mu^{\prime}}\left(\tilde{q}^{\prime}\right) q_{-\tilde{q}} q_{\tilde{q}^{\prime}} \Pi_{\mu \mu^{\prime}}^{z z}\left(\tilde{q}, \tilde{q}^{\prime}\right)\right.\right. \\
& \left.+\frac{1}{2} z_{\mu}(-\tilde{q}-\mathbf{Q}) z_{\mu^{\prime}}\left(\tilde{q}^{\prime}\right) p_{-\tilde{q}} q_{\tilde{q}^{\prime}} \Pi_{\mu \mu^{\prime}}^{y z}\left(\tilde{q}+\mathbf{Q}, \tilde{q}^{\prime}\right)+\frac{1}{2} z_{\mu}(-\tilde{q}) z_{\mu^{\prime}}\left(\tilde{q}^{\prime}+\mathbf{Q}\right) q_{-\tilde{q}} p_{\tilde{q}^{\prime}} \Pi_{\mu \mu^{\prime}}^{z y}\left(\tilde{q}, \tilde{q}^{\prime}+\mathbf{Q}\right)\right] \\
& +\sum_{\mu=0, x, y}\left[z_{\mu}\left(\tilde{q}^{\prime}+\mathbf{Q}\right) r_{-\tilde{q}} p_{\tilde{q}^{\prime}} \Pi_{0 \mu}^{x y}\left(\tilde{q}+\mathbf{Q}, \tilde{q}^{\prime}+\mathbf{Q}\right)+\frac{1}{2} z_{\mu}\left(\tilde{q}^{\prime}\right) r_{-\tilde{q}} q_{\tilde{q}^{\prime}} \Pi_{0 \mu}^{x z}\left(\tilde{q}+\mathbf{Q}, \tilde{q}^{\prime}\right)\right. \\
& +z_{\mu}(-\tilde{q}-\mathbf{Q}) p_{-\tilde{q}^{\prime}} r_{\tilde{q}^{\prime}} \Pi_{\mu 0}^{y x}\left(\tilde{q}+\mathbf{Q}, \tilde{q}^{\prime}+\mathbf{Q}\right)+\frac{1}{2} z_{\mu}(-\tilde{q}) q_{-\tilde{q}^{\prime}} r_{\tilde{q}^{\prime}} \Pi_{\mu 0}^{z x}\left(\tilde{q}, \tilde{q}^{\prime}+\mathbf{Q}\right) \\
& -\cos \theta_{0}\left(z_{\mu}\left(\tilde{q}^{\prime}+\mathbf{Q}\right) i \delta m_{-\tilde{q}}^{\mathrm{HS}} p_{\tilde{q}^{\prime}} \Pi_{0 \mu}^{z y}\left(\tilde{q}, \tilde{q}^{\prime}+\mathbf{Q}\right)+\frac{1}{2} z_{\mu}\left(\tilde{q}^{\prime}\right) i \delta m_{-\tilde{q}}^{\mathrm{HS}} q_{\tilde{q}^{\prime}} \Pi_{0 \mu}^{z z}\left(\tilde{q}, \tilde{q}^{\prime}\right)\right. \\
& \left.\left.+z_{\mu}(-\tilde{q}-\mathbf{Q}) p_{-\tilde{q}} i \delta m_{\tilde{q}^{\prime}}^{\mathrm{HS}} \Pi_{\mu 0}^{y z}\left(\tilde{q}+\mathbf{Q}, \tilde{q}^{\prime}\right)+\frac{1}{2} z_{\mu}(-\tilde{q}) q_{-\tilde{q}} i \delta m_{\tilde{q}^{\prime}}^{\mathrm{HS}} \Pi_{\mu 0}^{z z}\left(\tilde{q}, \tilde{q}^{\prime}\right)\right)\right] \\
& +r_{-\tilde{q}} r_{\tilde{q}^{\prime}} \Pi_{00}^{x x}\left(\tilde{q}+\mathbf{Q}, \tilde{q}^{\prime}+\mathbf{Q}\right)-\cos ^{2} \theta_{0} \delta m_{-\tilde{q}}^{\mathrm{HS}} \delta m_{\tilde{q}^{\prime}}^{\mathrm{HS}} \Pi_{00}^{z z}\left(\tilde{q}, \tilde{q}^{\prime}\right) \\
& \left.-\cos \theta_{0}\left[r_{-\tilde{q}} i \delta m_{\tilde{q}^{\prime}}^{\mathrm{HS}} \Pi_{00}^{x z}\left(\tilde{q}+\mathbf{Q}, \tilde{q}^{\prime}\right)+i \delta m_{-\tilde{q}}^{\mathrm{HS}} \tilde{q}_{\tilde{q}^{\prime}} \Pi_{00}^{z x}\left(\tilde{q}, \tilde{q}^{\prime}+\mathbf{Q}\right)\right]\right\}
\end{aligned}
$$

where $r_{\tilde{q}}=2 h_{0} p_{\tilde{q}}-\sin \theta_{0} i \delta m_{\tilde{q}}^{\mathrm{HS}}$. To proceed further, we use the expression of the correlation function $\Pi_{\mu \mu^{\prime}}^{\nu \nu^{\prime}}\left(\tilde{q}, \tilde{q}^{\prime}\right)$ obtained in Appendix A. We thus have $\tilde{q}=\tilde{q}^{\prime}$ in (B17). In order to obtain the effective action of the $q$ field to lowest order in $\partial_{\mu} q$, it is sufficient to retain the first-order derivative terms $\partial_{\mu} q$. Since $z_{\mu}(\tilde{q})=O\left(\partial_{\mu}\right)$, one can use $z_{0}(\tilde{q})=-\omega_{\nu}, z_{\mu \neq 0}(\tilde{q})=-i q_{\mu}, z_{0}(\tilde{q}+\mathbf{Q})=0, z_{\mu \neq 0}(\tilde{q}+\mathbf{Q})=2$, and evaluate the correlation functions $\Pi_{\mu \mu^{\prime}}^{\nu \nu^{\prime}}$ at $\tilde{q}=\tilde{q}^{\prime}=0$ in B17). This gives

$$
\begin{aligned}
\left\langle\left(S_{1}+S_{2}\right)^{2}\right\rangle_{c}= & \sum_{\tilde{q}}\left\{p_{-\tilde{q}} p_{\tilde{q}}\left(4 \sum_{\mu, \mu^{\prime} \neq 0} \Pi_{\mu \mu^{\prime}}^{y y}(\mathbf{Q}, \mathbf{Q})+4 h_{0}^{2} \Pi_{00}^{x x}(\mathbf{Q}, \mathbf{Q})+16 h_{0} \Pi_{0 x}^{x y}(\mathbf{Q}, \mathbf{Q})\right)\right. \\
& +q_{-\tilde{q}} q_{\tilde{q}}\left(-\frac{\omega_{\nu}^{2}}{4} \Pi_{00}^{z z}(0,0)+\frac{\mathbf{q}^{2}}{4} \Pi_{x x}^{z z}(0,0)\right), \\
& -\delta m_{-\tilde{q}}^{\mathrm{HS}} \delta m_{\tilde{q}}^{\mathrm{HS}}\left(\sin ^{2} \theta_{0} \Pi_{00}^{x x}(\mathbf{Q}, \mathbf{Q})+\cos ^{2} \theta_{0} \Pi_{00}^{z z}(0,0)+\sin \left(2 \theta_{0}\right) \Pi_{00}^{z x}(0, \mathbf{Q})\right) \\
& -\left[p_{-\tilde{q}} q_{\tilde{q}} \omega_{\nu}\left(2 \Pi_{0 x}^{z y}(0, \mathbf{Q})+h_{0} \Pi_{00}^{z x}(0, \mathbf{Q})\right)-\text { c.c. }\right] \\
& -i\left[p _ { - \tilde { q } } \delta m _ { \tilde { q } } ^ { \mathrm { HS } } \left(2 h_{0} \sin \theta_{0} \Pi_{00}^{x x}(\mathbf{Q}, \mathbf{Q})+4 \sin \theta_{0} \Pi_{0 x}^{x y}(\mathbf{Q}, \mathbf{Q})\right.\right. \\
& \left.\left.+4 \cos \theta_{0} \Pi_{0 x}^{z y}(0, \mathbf{Q})+2 h_{0} \cos \theta_{0} \Pi_{00}^{z x}(0, \mathbf{Q})\right)+ \text { c.c. }\right] \\
& \left.-i\left[q_{-\tilde{q}} \delta m_{\tilde{q}}^{\mathrm{HS}} \frac{\omega_{\nu}}{2}\left(\sin \theta_{0} \Pi_{00}^{z x}(0, \mathbf{Q})+\cos \theta_{0} \Pi_{00}^{z z}(0,0)\right)-\text { c.c. }\right]\right\} .
\end{aligned}
$$

A comment is in order here. The first-order cumulant (B6) gives a term linear in $q_{\mathbf{r}}$ :

$$
\delta S_{B}=\frac{i}{2} x \int_{0}^{\beta} d \tau \sum_{\mathbf{r}} \dot{q}_{\mathbf{r}}
$$


This term comes from the Berry phase term $S_{B}=\int_{0}^{\beta} d \tau \sum_{\mathbf{r}}\left\langle\phi_{\mathbf{r}}^{\dagger} R_{\mathbf{r}}^{\dagger} \dot{R}_{\mathbf{r}} \phi_{\mathbf{r}}\right\rangle$. The $\mathrm{SU}(2)$ matrix $R_{\mathbf{r}}$ is defined up to the U(1) gauge transformation $R_{\mathbf{r}} \rightarrow R_{\mathbf{r}} e^{-\frac{i}{2} \psi_{\mathbf{r}} \sigma \cdot \Omega_{\mathbf{r}}^{\mathrm{cl}}}$. The Berry phase term depends on the gauge choice ${ }^{38}$ The gauge-dependent term is given by

$$
-\frac{i}{2} \int_{0}^{\beta} d \tau \sum_{\mathbf{r}}\left\langle\phi_{\mathbf{r}}^{\dagger} \boldsymbol{\sigma} \cdot \boldsymbol{\Omega}_{\mathbf{r}}^{\mathrm{cl}} \phi_{\mathbf{r}}\right\rangle \dot{\psi}_{\mathbf{r}}=-\frac{i}{2} m_{0} \int_{0}^{\beta} d \tau \sum_{\mathbf{r}} \dot{\psi}_{\mathbf{r}}
$$

In the following we take $\psi_{\mathbf{r}}=\varphi_{\mathbf{r}} / m_{0}$. As shown in Sec. IC this choice ensures that in the attractive model, half the fermion density is identified as the conjugate variable of the phase $\Theta$ of the superconducting order parameter, as required by gauge invariance ${ }^{34}$ Including the gauge-dependent term of $S_{B}$ [Eq. (B20]) in $\delta S_{B}$, we obtain

$$
\delta S_{B}=-\frac{i}{2} \rho_{0} \int_{0}^{\beta} d \tau \sum_{\mathbf{r}} \dot{q}_{\mathbf{r}}
$$

From Eqs. (B6]B8181821), we then obtain

$$
\begin{aligned}
S\left[p, q, m^{\mathrm{HS}}, m\right]= & \frac{1}{2} \sum_{\tilde{q}}\left[p_{-\tilde{q}} \Pi_{p p}(\tilde{q}) p_{\tilde{q}}+q_{-\tilde{q}} \Pi_{q q}(\tilde{q}) q_{\tilde{q}}+\delta m_{-\tilde{q}}^{\mathrm{HS}} \Pi_{m^{\mathrm{HS}} m^{\mathrm{HS}}}(\tilde{q}) \delta m_{\tilde{q}}^{\mathrm{HS}}+2 p_{-\tilde{q}} \Pi_{p q}(\tilde{q}) q_{\tilde{q}}\right. \\
& \left.+2 p_{-\tilde{q}} \Pi_{p m^{\mathrm{HS}}}(\tilde{q}) \delta m_{\tilde{q}}^{\mathrm{HS}}+2 q_{-\tilde{q}} \Pi_{q m^{\mathrm{HS}}}(\tilde{q}) \delta m_{\tilde{q}}^{\mathrm{HS}}+2 i \delta m_{-\tilde{q}} \delta m_{\tilde{q}}^{\mathrm{HS}}-\frac{U}{2} \delta m_{-\tilde{q}} \delta m_{\tilde{q}}\right]+\delta S_{B}
\end{aligned}
$$

where

$$
\begin{aligned}
\Pi_{p p}(\tilde{q}) & =-4 \sum_{\mu, \mu^{\prime} \neq 0} \Pi_{\mu \mu^{\prime}}^{y y}(\mathbf{Q}, \mathbf{Q})-4 h_{0}^{2} \Pi_{00}^{x x}(\mathbf{Q}, \mathbf{Q})-16 h_{0} \Pi_{0 x}^{x y}(\mathbf{Q}, \mathbf{Q})-4 x h_{0}+4\langle-K\rangle, \\
\Pi_{q q}(\tilde{q}) & =\frac{\omega_{\nu}^{2}}{4} \Pi_{00}^{z z}(0,0)+\frac{\mathbf{q}^{2}}{8}\langle-K\rangle, \\
\Pi_{m^{\mathrm{HS}} m^{\mathrm{HS}}}(\tilde{q}) & =\sin ^{2} \theta_{0} \Pi_{00}^{x x}(\mathbf{Q}, \mathbf{Q})+\cos ^{2} \theta_{0} \Pi_{00}^{z z}(0,0)+\sin \left(2 \theta_{0}\right) \Pi_{00}^{z x}(0, \mathbf{Q}), \\
\Pi_{p q}(\tilde{q}) & =\omega_{\nu}\left[2 \Pi_{0 x}^{z y}(0, \mathbf{Q})+h_{0} \Pi_{00}^{z x}(0, \mathbf{Q})+2 \Delta_{0}\right], \\
\Pi_{p m^{\mathrm{HS}}}(\tilde{q}) & =2 i\left[h_{0} \sin \theta_{0} \Pi_{00}^{x x}(\mathbf{Q}, \mathbf{Q})+2 \sin \theta_{0} \Pi_{0 x}^{x y}(\mathbf{Q}, \mathbf{Q})+2 \cos \theta_{0} \Pi_{0 x}^{z y}(0, \mathbf{Q})+h_{0} \cos \theta_{0} \Pi_{00}^{z x}(0, \mathbf{Q})\right], \\
\Pi_{q m^{\mathrm{HS}}}(\tilde{q}) & =\frac{i}{2} \omega_{\nu}\left[\sin \theta_{0} \Pi_{00}^{z x}(0, \mathbf{Q})+\cos \theta_{0} \Pi_{00}^{z z}(0,0)\right] .
\end{aligned}
$$

$p_{\tilde{q}}, q_{\tilde{q}}, \delta m_{\tilde{q}}^{\mathrm{HS}}$ and $\delta m_{\tilde{q}}$ are the Fourier transformed fields of $p_{\mathbf{r}}, q_{\mathbf{r}}, \delta m_{\mathbf{r}}^{\mathrm{HS}}$ and $\delta m_{\mathbf{r}} . \tilde{q}=\left(\mathbf{q}, i \omega_{\nu}\right)$ where $\omega_{\nu}=\nu 2 \pi T$ $(\nu$ integer) is a bosonic Matsubara frequency. Eqs. (B23) are valid in the hydrodynamic regime $(\tilde{q} \rightarrow 0)$. They are sufficient to obtain the effective action of the AF field $q$ to order $O\left(\partial_{\mu}^{2}\right)$.

Integrating out the Hubbard-Stratonovich field $m^{\mathrm{HS}}$, we obtain Eq. (34) with

$$
\begin{aligned}
& \tilde{\Pi}_{p p}=\Pi_{p p}-\frac{\Pi_{p m^{\mathrm{HS}}} \Pi_{m^{\mathrm{HS}} p}}{\Pi_{m^{\mathrm{HS}} m^{\mathrm{HS}}}}, \\
& \tilde{\Pi}_{q q}=\Pi_{q q}-\frac{\Pi_{q m^{\mathrm{HS}}} \Pi_{m^{\mathrm{HS}} q}}{\Pi_{m^{\mathrm{HS}} m^{\mathrm{HS}}}}, \\
& \tilde{\Pi}_{m m}=\frac{1}{\Pi_{m^{\mathrm{HS}} m^{\mathrm{HS}}}}-\frac{U}{2}, \\
& \tilde{\Pi}_{p q}=\Pi_{p q}-\frac{\Pi_{p m^{\mathrm{HS}}} \Pi_{m^{\mathrm{HS}} q}}{\Pi_{m^{\mathrm{HS}} m^{\mathrm{HS}}}}, \\
& \tilde{\Pi}_{p m}=-i \frac{\Pi_{p m^{\mathrm{HS}}}}{\Pi_{m^{\mathrm{HS}} m^{\mathrm{HS}}}}, \\
& \tilde{\Pi}_{q m}=-i \frac{\Pi_{q m^{\mathrm{HS}}}}{\Pi_{m^{\mathrm{HS}} m^{\mathrm{HS}}}} .
\end{aligned}
$$

The action $S[p, q, m]$ takes a very simple form in the weak-coupling (Slater) and strong-coupling (Mott-Heisenberg) limits. 


\section{Slater limit}

We assume that we are not too close to half-filling so that the zero-temperature order parameter $\Delta_{0}^{\mathrm{HS}}$ is given by (26). Since $\Delta_{0}^{\mathrm{HS}}$ is exponentially small at weak coupling, $\theta_{0} \simeq \pi$ and $i m_{0}^{\mathrm{HS}} \simeq x U / 2$ [see Eqs. (1521)]. We also have

$$
\begin{aligned}
\Pi_{00}^{z x}(0, \mathbf{Q}) & =\Delta_{0}^{\mathrm{HS}} \int_{\mathbf{k}} \frac{\epsilon_{\mathbf{k} \uparrow}}{E_{\mathbf{k} \uparrow}^{3}} \\
& \simeq \Delta_{0}^{\mathrm{HS}} \mathcal{N}_{0}\left(\epsilon_{F}\right) \int_{-4 t}^{4 t} d \epsilon \frac{\epsilon-\epsilon_{F}}{\left[\left(\epsilon-\epsilon_{F}\right)^{2}+\Delta_{0}^{\mathrm{HS}}\right]^{3 / 2}} \\
& \simeq 0 .
\end{aligned}
$$

Since the integral in (B25) is peaked around $\epsilon=\epsilon_{F}$ for $\Delta_{0}^{\mathrm{HS}} \rightarrow 0$, we have replaced the density of states $\mathcal{N}_{0}(\epsilon)$ by its value at the Fermi energy $\epsilon_{F}$ [see the discussion after Eq. (25)]. For the same reason, we can extend the integration range to ] $-\infty, \infty$ [, so that the integral vanishes. This result is a consequence of the particle-hole symmetry which holds in the weak-coupling (BCS) limit of the attractive model. Similarly, we find $\Pi_{0 x}^{z y}(0, \mathbf{Q}) \simeq 0$. We therefore have

$$
\begin{aligned}
\Pi_{q q} & =\frac{\Pi_{00}^{z z}(0,0)}{4} \omega_{\nu}^{2}+\frac{\langle-K\rangle}{8} \mathbf{q}^{2}, \\
\Pi_{m^{\mathrm{HS}} m^{\mathrm{HS}}} & =\Pi_{00}^{z z}(0,0) \\
\Pi_{p q}(\tilde{q}) & =2 \omega_{\nu} \Delta_{0} \simeq 0 \\
\Pi_{p m^{\mathrm{HS}}}(\tilde{q}) & =0 \\
\Pi_{q m^{\mathrm{HS}}}(\tilde{q}) & =-\frac{i}{2} \Pi_{00}^{z z}(0,0) \omega_{\nu} .
\end{aligned}
$$

Using Eqs. (B24, we deduce

$$
\begin{aligned}
\tilde{\Pi}_{q q} & =\frac{\langle-K\rangle}{8} \mathbf{q}^{2}, \\
\tilde{\Pi}_{m m} & =\frac{1}{\Pi_{00}^{z z}(0,0)}-\frac{U}{2}, \\
\tilde{\Pi}_{q m} & =-\frac{\omega_{\nu}}{2}
\end{aligned}
$$

and $\tilde{\Pi}_{p q}=\tilde{\Pi}_{p m}=0 . p$ fluctuations do not couple to $q$ and $m$ fluctuations. We therefore obtain the effective action

$$
\begin{aligned}
S[q, m]= & \frac{1}{2} \int_{0}^{\beta} d \tau \int d^{2} r\left[i \delta m_{\mathbf{r}} \dot{q}_{\mathbf{r}}+\frac{\langle-K\rangle}{8}\left(\nabla q_{\mathbf{r}}\right)^{2}\right. \\
& \left.+\left(\frac{1}{\Pi_{00}^{z z}(0,0)}-\frac{U}{2}\right) \delta m_{\mathbf{r}}^{2}\right]+\delta S_{B},
\end{aligned}
$$

where we have taken the continuum limit in real space.

\section{Mott-Heisenberg limit}

In the strong-coupling limit, there are well-defined local moments with a fixed amplitude so that we can ignore the fluctuations of $m^{\mathrm{HS}}$ and $m$. Low-energy fluctuations correspond to direction fluctuations of these local moments. This can be seen explicitly by integrating out the $m$ field in the action $S\left[p, q, m^{\mathrm{HS}}, m\right]$ [Eq. (B22)]. This yields the replacement

$$
\Pi_{m^{\mathrm{HS}} m^{\mathrm{HS}}} \rightarrow \Pi_{m^{\mathrm{HS}} m^{\mathrm{HS}}}^{\prime}=\Pi_{m^{\mathrm{HS}} m^{\mathrm{HS}}}-\frac{2}{U} .
$$

To leading order in $1 / U$, we have [see Eqs. A8 in Appendix

$$
\begin{aligned}
\Pi_{m^{\mathrm{HS}} m^{\mathrm{HS}}}^{\prime}(\tilde{q}) & =-\frac{2}{U}, \\
\Pi_{p m^{\mathrm{HS}}} & =O\left(\frac{t^{2}}{U^{2}}\right), \\
\Pi_{q m^{\mathrm{HS}}} & =O\left(\frac{\omega_{\nu} t}{U^{2}}\right) .
\end{aligned}
$$


Integrating out $m^{\mathrm{HS}}$ yields the correction terms

$$
\begin{aligned}
& -\frac{\Pi_{p m^{\mathrm{HS}}} \Pi_{m^{\mathrm{HS}} p}}{\Pi_{m^{\mathrm{HS}} m^{\mathrm{HS}}}^{\prime}}=O\left(\frac{t^{4}}{U^{3}}\right), \\
& -\frac{\Pi_{q m^{\mathrm{HS}}} \Pi_{m^{\mathrm{HS}}} q}{\Pi_{m^{\mathrm{HS}}}^{\prime} m^{\mathrm{HS}}}=O\left(\frac{\omega_{\nu}^{2} t^{2}}{U^{3}}\right), \\
& -\frac{\Pi_{p m^{\mathrm{HS}}} \Pi_{m^{\mathrm{HS}}} q}{\Pi_{m^{\mathrm{HS}}}^{\prime} m^{\mathrm{HS}}}=O\left(\frac{\omega_{\nu} t^{3}}{U^{3}}\right),
\end{aligned}
$$

to $\Pi_{p p}, \Pi_{q q}$ and $\Pi_{p q}$, respectively. These terms can be ignored in the limit $U \gg 4 t$.

Using Eqs. A8 of Appendix A1 we therefore have

$$
\begin{aligned}
& \Pi_{p p}(\tilde{q})=8 J \sin ^{2} \theta_{0}, \\
& \Pi_{q q}(\tilde{q})=\mathbf{q}^{2} \frac{J}{4} \sin ^{2} \theta_{0}, \\
& \Pi_{p q}(\tilde{q})=\omega_{\nu} \sin \theta_{0},
\end{aligned}
$$

and $\Pi_{p m^{\mathrm{HS}}}=\Pi_{q m^{\mathrm{HS}}} \simeq 0$. Using then $\tilde{\Pi}=\Pi$ [Eqs. (B24)], we obtain the effective action

$$
\begin{aligned}
S[p, q]= & \frac{1}{2} \int_{0}^{\beta} d \tau \int d^{2} r\left[2 i \sqrt{1-x^{2}} p_{\mathbf{r}} \dot{q}_{\mathbf{r}}\right. \\
& \left.+\frac{J}{4}\left(1-x^{2}\right)\left(\nabla q_{\mathbf{r}}\right)^{2}+8 J\left(1-x^{2}\right) p_{\mathbf{r}}^{2}\right]+\delta S_{B} .
\end{aligned}
$$

\section{APPENDIX C: KINETIC ENERGY IN THE HF STATE $(T=0)$}

In this Appendix, we derive Eq. (56). We start from

$$
\begin{aligned}
\nabla_{\mathbf{k}} \cdot\left(\frac{\epsilon_{\mathbf{k} \uparrow}}{E_{\mathbf{k} \uparrow}} \mathbf{v}_{\mathbf{k}}\right) & =\frac{\epsilon_{\mathbf{k} \uparrow}}{E_{\mathbf{k} \uparrow}} \nabla_{\mathbf{k}} \cdot \mathbf{v}_{\mathbf{k}}+\nabla_{\mathbf{k}}\left(\frac{\epsilon_{\mathbf{k} \uparrow}}{E_{\mathbf{k} \uparrow}}\right) \cdot \mathbf{v}_{\mathbf{k}} \\
& =-\frac{\epsilon_{\mathbf{k} \uparrow} \epsilon_{\mathbf{k}}}{E_{\mathbf{k} \uparrow}}+\frac{\Delta_{0}^{\mathrm{HS}}}{E_{\mathbf{k} \uparrow}^{3}} v_{\mathbf{k}}^{2} .
\end{aligned}
$$

where $\mathbf{v}_{\mathbf{k}}=\nabla_{\mathbf{k}} \epsilon_{\mathbf{k}}$. Integrating the left hand side of this equation over the entire Brillouin zone, we obtain

$$
\int_{\mathbf{k}} \nabla_{\mathbf{k}} \cdot\left(\frac{\epsilon_{\mathbf{k} \uparrow}}{E_{\mathbf{k} \uparrow}} \mathbf{v}_{\mathbf{k}}\right)=\oint \frac{\epsilon_{\mathbf{k} \uparrow}}{E_{\mathbf{k} \uparrow}} \mathbf{v}_{\mathbf{k}} \cdot d \mathbf{l}_{\mathbf{k}}=0,
$$

where the contour in the last integral is given by the Brillouin zone (BZ) boundary and $d \mathbf{l}_{\mathbf{k}}$ is perpendicular to the contour. The integral vanishes since $\mathbf{v}_{\mathbf{k}} \cdot d \mathbf{l}_{\mathbf{k}}=0$ at the BZ boundary. We deduce from (C1 C2

$$
\langle-K\rangle_{T=0}=\int_{\mathbf{k}} \frac{\epsilon_{\mathbf{k} \uparrow} \epsilon_{\mathbf{k}}}{E_{\mathbf{k} \uparrow}}=\Delta_{0}^{\mathrm{HS}^{2}} \int_{\mathbf{k}} \frac{v_{\mathbf{k}}^{2}}{E_{\mathbf{k} \uparrow}^{3}} .
$$

In the weak-coupling limit, using $\Delta_{0}^{\mathrm{HS}^{2}} / E_{\mathbf{k} \uparrow}^{3} \equiv 2 \delta\left(\epsilon_{\mathbf{k}}-\epsilon_{F}\right)$, we obtain (56).

\section{APPENDIX D: CHARGE $\left(\rho_{\mathbf{r}}\right)$ AND PAIRING $\left(\Delta_{\mathrm{r}}\right)$ FIELDS}

In the section, we relate the charge $\left(\rho_{\mathbf{r}}\right)$ and pairing $\left(\Delta_{\mathbf{r}}\right)$ fluctuations of the attractive model to the fields $p, q$ and $\delta m$ defined in the repulsive model. We rewrite $S_{J}[\mathrm{Eq}$. (35)] as

$$
S_{J}=\int_{0}^{\beta} d \tau \sum_{\mathbf{r}} \phi_{\mathbf{r}}^{\dagger} \mathbf{B}_{0 \mathbf{r}} \phi_{\mathbf{r}}
$$


where $\mathbf{B}_{0 \mathbf{r}}=R_{\mathbf{r}}^{\dagger} \mathbf{J}_{\mathbf{r}} \cdot \boldsymbol{\sigma} R_{\mathbf{r}}=\sum_{\nu=x, y, z} B_{0 \mathbf{r}}^{\nu} \sigma^{\nu}$ and

$$
\begin{aligned}
& B_{0 \mathbf{r}}^{x}=(-1)^{\mathbf{r}} \cos \left(\theta_{\mathbf{r}}-\theta_{0}\right)\left[\cos \varphi_{\mathbf{r}} J_{\mathbf{r}}^{x}+\sin \varphi_{\mathbf{r}} J_{\mathbf{r}}^{y}\right]-(-1)^{\mathbf{r}} \sin \left(\theta_{\mathbf{r}}-\theta_{0}\right) J_{\mathbf{r}}^{z}, \\
& B_{0 \mathbf{r}}^{y}=-(-1)^{\mathbf{r}} \sin \varphi_{\mathbf{r}} J_{\mathbf{r}}^{x}+(-1)^{\mathbf{r}} \cos \varphi_{\mathbf{r}} J_{\mathbf{r}}^{y}, \\
& B_{0 \mathbf{r}}^{z}=\sin \left(\theta_{\mathbf{r}}-\theta_{0}\right)\left[\cos \varphi_{\mathbf{r}} J_{\mathbf{r}}^{x}+\sin \varphi_{\mathbf{r}} J_{\mathbf{r}}^{y}\right]+\cos \left(\theta_{\mathbf{r}}-\theta_{0}\right) J_{\mathbf{r}}^{z} .
\end{aligned}
$$

To first order in $\mathbf{J}$ and second-order in $p, \delta m^{\mathrm{HS}}, \delta m$, the effective action is given by $\left\langle S_{1}+S_{2}+S_{J}\right\rangle-\frac{1}{2}\left\langle\left(S_{1}+S_{2}+S_{J}\right)^{2}\right\rangle_{c}$. The first-order cumulant gives the source-dependent contribution

$$
S_{J}^{(1)}=\int_{0}^{\beta} d \tau \sum_{\mathbf{r}}\left[2 \Delta_{0}(-1)^{\mathbf{r}} B_{0 \mathbf{r}}^{x}-x B_{0 \mathbf{r}}^{z}\right]
$$

From the second-order cumulant, we obtain to linear order in the source

$$
\begin{aligned}
S_{J}^{(2)}= & -\int_{0}^{\beta} d \tau d \tau^{\prime} \sum_{\substack{\mathbf{r}, \mathbf{r}^{\prime} \\
\nu, \nu^{\prime}=x, z \\
\mu^{\prime}=0, x, y}} B_{0 \mathbf{r}}^{\nu}(\tau) \Pi_{0 \mu^{\prime}}^{\nu \nu^{\prime}}\left(\mathbf{r}, \tau ; \mathbf{r}^{\prime}, \tau^{\prime}\right) A_{\mu^{\prime} \mathbf{r}^{\prime}}^{\nu(\mathrm{tot})}\left(\tau^{\prime}\right) \\
= & -\int_{0}^{\beta} d \tau \sum_{\mathbf{r}}\left\{(-1)^{\mathbf{r}} B_{0 \mathbf{r}}^{x}\left[4 \Pi_{0 x}^{x y}(\mathbf{Q}, \mathbf{Q}) p_{\mathbf{r}}+\Pi_{00}^{x x}(\mathbf{Q}, \mathbf{Q})\left(2 h_{0} p_{\mathbf{r}}-\sin \theta_{0} i \delta m_{\mathbf{r}}^{\mathrm{HS}}\right)+\Pi_{00}^{z x}(0, \mathbf{Q})\left(-\frac{i}{2} \dot{q}_{\mathbf{r}}-\cos \theta_{0} i \delta m_{\mathbf{r}}^{\mathrm{HS}}\right)\right]\right. \\
& \left.+B_{0 \mathbf{r}}^{z}\left[4 \Pi_{0 x}^{z y}(0, \mathbf{Q}) p_{\mathbf{r}}+\Pi_{00}^{z x}(0, \mathbf{Q})\left(2 h_{0} p_{\mathbf{r}}-\sin \theta_{0} i \delta m_{\mathbf{r}}^{\mathrm{HS}}\right)+\Pi_{00}^{z z}(0,0)\left(-\frac{i}{2} \dot{q}_{\mathbf{r}}-\cos \theta_{0} i \delta m_{\mathbf{r}}^{\mathrm{HS}}\right)\right]\right\},
\end{aligned}
$$

where the last expression is valid in the hydrodynamic regime. $A_{\mu}^{\nu(\text { tot })}$ is defined in (B11). In the presence of the source $\mathbf{J}$, the effective action $S\left[p, q, m^{\mathrm{HS}}, m\right]$ [Eq. (B22)] should be supplemented with $S_{J}^{(1)}+S_{J}^{(2)}$. The integration of the $m^{\mathrm{HS}}$ field then leads to the source-dependent action $S_{J}^{(1)}+S_{J}^{(2)^{\prime}}+S_{J}^{(3)}$ where $S_{J}^{(2)^{\prime}}=\left.S_{J}^{(2)}\right|_{\delta m^{\mathrm{HS}}=0}$ and

$$
\begin{aligned}
S_{J}^{(3)}= & -\frac{i}{\Pi_{m^{\mathrm{HS}} m^{\mathrm{HS}}}} \int_{0}^{\beta} d \tau \sum_{\mathbf{r}}\left\{(-1)^{\mathbf{r}} B_{0 \mathbf{r}}^{x}\left[\sin \theta_{0} \Pi_{00}^{x x}(\mathbf{Q}, \mathbf{Q})+\cos \theta_{0} \Pi_{00}^{z x}(0, \mathbf{Q})\right]\right. \\
& \left.+B_{0 \mathbf{r}}^{z}\left[\sin \theta_{0} \Pi_{00}^{z x}(0, \mathbf{Q})+\cos \theta_{0} \Pi_{00}^{z z}(0,0)\right]\right\}\left(\Pi_{m^{\mathrm{HS}} p} p_{\mathbf{r}}+\Pi_{m^{\mathrm{HS}} q}^{r} i \dot{q}_{\mathbf{r}}+i \delta m_{\mathbf{r}}\right) .
\end{aligned}
$$

We have introduced $\Pi_{m^{\mathrm{HS}} q}^{r}=\Pi_{m^{\mathrm{HS}} q} / \omega_{\nu}$. In Eq. (D5), the correlation functions $\Pi_{m^{\mathrm{HS}} m^{\mathrm{HS}}, \Pi_{m}{ }_{\mathrm{HS}}, \Pi_{m}^{r}{ }^{\mathrm{HS}} q}$ are evaluated at $\tilde{q}=0$. Taking the functional derivative of $S_{J}^{(1)}+S_{J}^{(2)^{\prime}}+S_{J}^{(3)}$ with respect to $\mathbf{J}$, we finally obtain

$$
\begin{aligned}
\delta \rho_{\mathbf{r}}= & p_{\mathbf{r}}\left[-4 \Delta_{0}-2 h_{0} \Pi_{00}^{z x}(0, \mathbf{Q})-4 \Pi_{0 x}^{z y}(0, \mathbf{Q})\right]+\Pi_{00}^{z z}(0,0) \frac{i}{2} \dot{q}_{\mathbf{r}} \\
& -\frac{i}{\Pi_{m^{\mathrm{HS}} m^{\mathrm{HS}}}}\left[\sin \theta_{0} \Pi_{00}^{z x}(0, \mathbf{Q})+\cos \theta_{0} \Pi_{00}^{z z}(0,0)\right]\left(\Pi_{m^{\mathrm{HS}}} p_{\mathbf{r}}+\Pi_{m^{\mathrm{HS}} q}^{r} i \dot{q}_{\mathbf{r}}+i \delta m_{\mathbf{r}}\right), \\
\delta\left|\Delta_{\mathbf{r}}\right|= & p_{\mathbf{r}}\left[-x-h_{0} \Pi_{00}^{x x}(\mathbf{Q}, \mathbf{Q})-2 \Pi_{0 x}^{x y}(\mathbf{Q}, \mathbf{Q})\right]+\Pi_{00}^{z x}(0, \mathbf{Q}) \frac{i}{4} \dot{q}_{\mathbf{r}} \\
& -\frac{i}{2 \Pi_{m^{\mathrm{HS}} m^{\mathrm{HS}}}}\left[\sin \theta_{0} \Pi_{00}^{x x}(\mathbf{Q}, \mathbf{Q})+\cos \theta_{0} \Pi_{00}^{z x}(0, \mathbf{Q})\right]\left(\Pi_{m^{\mathrm{HS}} p} p_{\mathbf{r}}+\Pi_{m^{\mathrm{HS}}}^{r} i \dot{q}_{\mathbf{r}}+i \delta m_{\mathbf{r}}\right), \\
\Theta_{\mathbf{r}}= & -q_{\mathbf{r}}
\end{aligned}
$$

\section{Strong-coupling limit}

In the strong-coupling limit, we can obtain a simple relation between $\rho_{\mathbf{r}}, \Delta_{\mathbf{r}}$ and $m_{\mathbf{r}} \boldsymbol{\Omega}_{\mathbf{r}}$. In Sec. IV we show that the Hubbard model reduces to the Heisenberg model when $U \gg 4 t$. We can carry out the same derivation in the presence of the source term (D1). Integrating out the fermions, we obtain the source-dependent term

$$
S_{J}=\int_{0}^{\beta} d \tau \sum_{\mathbf{r}}\left\langle\phi_{\mathbf{r}}^{\dagger} R_{\mathbf{r}}^{\dagger} \mathbf{J}_{\mathbf{r}} \cdot \boldsymbol{\sigma} R_{\mathbf{r}} \phi_{\mathbf{r}}\right\rangle_{\mathrm{at}}
$$


to leading order in $1 / U$. The average in (D7) is taken with respect to the atomic action 866). The matrix $R_{\mathbf{r}}$ satisfies $R_{\mathbf{r}} \sigma^{z} R_{\mathbf{r}}^{\dagger}=\boldsymbol{\sigma} \cdot \boldsymbol{\Omega}_{\mathbf{r}}$ (see Sec. IVA). Using $\left\langle\phi_{\mathbf{r}}^{\dagger} \sigma^{\nu} \phi_{\mathbf{r}}\right\rangle_{\text {at }}=\delta_{\nu, z}$ and $R_{\mathbf{r}}^{\dagger} \mathbf{J}_{\mathbf{r}} \cdot \boldsymbol{\sigma} R_{\mathbf{r}}=\boldsymbol{\sigma} \cdot \mathcal{R}_{\mathbf{r}}^{-1} \mathbf{J}_{\mathbf{r}}$, where

$$
\mathcal{R}_{\mathbf{r}}=\left(\begin{array}{ccc}
\cos \theta_{\mathbf{r}} \cos \varphi_{\mathbf{r}} & -\sin \varphi_{\mathbf{r}} & \sin \theta_{\mathbf{r}} \cos \varphi_{\mathbf{r}} \\
\cos \theta_{\mathbf{r}} \sin \varphi_{\mathbf{r}} & \cos \varphi_{\mathbf{r}} & \sin \theta_{\mathbf{r}} \sin \varphi_{\mathbf{r}} \\
-\sin \theta_{\mathbf{r}} & 0 & \cos \theta_{\mathbf{r}}
\end{array}\right)
$$

is the $\mathrm{SO}(3)$ rotation matrix which maps $\hat{\mathbf{z}}$ onto $\boldsymbol{\Omega}_{\mathbf{r}}$, we obtain

$$
S_{J}=\int_{0}^{\beta} d \tau \sum_{\mathbf{r}} \mathbf{J}_{\mathbf{r}} \cdot \boldsymbol{\Omega}_{\mathbf{r}}
$$

From Eqs. (36) we then deduce

$$
\begin{aligned}
\rho_{\mathbf{r}}-1 & =\Omega_{\mathbf{r}}^{z}, \\
\Delta_{\mathbf{r}} & =\frac{(-1)^{\mathbf{r}}}{2} \Omega_{\mathbf{r}}^{-},
\end{aligned}
$$

where $\Omega_{\mathbf{r}}^{ \pm}=\Omega_{\mathbf{r}}^{x} \pm i \Omega_{\mathbf{r}}^{y}$.

APPENDIX E: HF CUMULANTS $\left\langle S_{p}+S_{h_{0}}+S_{\delta m}\right\rangle$ AND $\left\langle\left(S_{h_{0}}+S_{\delta m}\right)^{2}+2\left(S_{h_{0}}+S_{\delta m}\right)\left(S_{p}+S_{l}\right)\right\rangle_{c}$

In this appendix, we calculate $\left\langle S_{p}+S_{h_{0}}+S_{\delta m}\right\rangle$ and $\left\langle\left(S_{h_{0}}+S_{\delta m}\right)^{2}+2\left(S_{h_{0}}+S_{\delta m}\right)\left(S_{p}+S_{l}\right)\right\rangle_{c}$ (Sec. III). The first-order cumulants read

$$
\begin{aligned}
\left\langle S_{p}\right\rangle & =-\int_{0}^{\beta} d \tau \sum_{\mathbf{r}} A_{0 \mathbf{r}}^{z}\left\langle j_{0 \mathbf{r}}^{z}\right\rangle \\
& =-i \frac{m_{0}}{U} \int_{0}^{\beta} d \tau \sum_{\mathbf{r}}(-1)^{\mathbf{r}}\left(\dot{\varphi}_{\mathbf{r}} \cos \theta_{\mathbf{r}}+\dot{\psi}_{\mathbf{r}}\right), \\
\left\langle S_{h_{0}}\right\rangle & =-h_{0} \int_{0}^{\beta} d \tau \sum_{\mathbf{r}} B_{0 \mathbf{r}}^{z}\left\langle j_{0 \mathbf{r}}^{z}\right\rangle \\
& =-\frac{2 h_{0} m_{0}}{U} \int_{0}^{\beta} d \tau \sum_{\mathbf{r}}(-1)^{\mathbf{r}} n_{\mathbf{r}}^{z},
\end{aligned}
$$

and

$$
\begin{aligned}
\left\langle S_{\delta m}\right\rangle & =-\int_{0}^{\beta} d \tau \sum_{\mathbf{r}}(-1)^{\mathbf{r}} \delta m_{\mathbf{r}}\left\langle j_{0 \mathbf{r}}^{z}\right\rangle \\
& =-\frac{2 m_{0}}{U} \int_{0}^{\beta} d \tau \sum_{\mathbf{r}} \delta m_{\mathbf{r}},
\end{aligned}
$$

where we have used $n_{\mathbf{r}}^{z}=\cos \theta_{\mathbf{r}}$ and the saddle-point equation $2 m_{0} / U=(-1)^{\mathbf{r}}\left\langle c_{\mathbf{r}}^{\dagger} \sigma^{z} c_{\mathbf{r}}\right\rangle$. Since $\mathbf{n}_{\mathbf{r}}$ is slowly varying, $\left\langle S_{h_{0}}\right\rangle$ vanishes. From (E2 69), we conclude that there is no linear contribution in $\delta m$.

Let us now consider the second-order cumulant

$$
\begin{aligned}
\left\langle S_{h_{0}} S_{\delta m}\right\rangle & =h_{0} \int_{0}^{\beta} d \tau d \tau^{\prime} \sum_{\substack{\mathbf{r}, \mathbf{r}^{\prime} \\
\nu=x, y, z}} B_{0 \mathbf{r}}^{\nu}(\tau) \Pi_{00}^{\nu z}\left(\mathbf{r}, \tau ; \mathbf{r}^{\prime}, \tau^{\prime}\right)(-1)^{\mathbf{r}^{\prime}} \delta m_{\mathbf{r}^{\prime}} \\
& =h_{0} \sum_{\substack{\tilde{q}, \tilde{q}^{\prime} \\
\nu=x, y, z}} B_{0}^{\nu}(-\tilde{q}) \Pi_{00}^{\nu z}\left(\tilde{q}, \tilde{q}^{\prime}\right) \delta m_{\tilde{q}^{\prime}+\mathbf{Q} .}
\end{aligned}
$$

$\Pi_{\mu \mu^{\prime}}^{\nu \nu^{\prime}}$ is the HF current-current correlation function for $h_{0}=0$ and an AF order parallel to the $z$ axis (i.e. $\Omega_{\mathbf{r}}^{\mathrm{cl}}=$ $\left.(-1)^{\mathbf{r}} \hat{\mathbf{z}}\right)$. It is given in Appendix 12 Since $B_{0 \mathbf{r}}^{\nu}$ is slowly varying, we can evaluate $\Pi_{\mu \mu^{\prime}}^{\nu \nu^{\prime}}\left(\tilde{q}, \tilde{q}^{\prime}\right)$ at $\tilde{q}=0$ in order to 
obtain the result to second order in $h_{0}, \partial_{\mu}$ and $\delta m$. Since $\Pi_{00}^{\nu z}\left(\tilde{q}=0, \tilde{q}^{\prime}\right)=0$ (Appendix $\left.\mathbf{A 2}_{2}\right),\left\langle S_{h_{0}} S_{\delta m}\right\rangle$ vanishes. A similar calculation shows that $\left\langle S_{p} S_{\delta m}\right\rangle=\left\langle S_{l} S_{\delta m}\right\rangle=0$. We therefore conclude that amplitude fluctuations decouple in the limit of a weak magnetic field.

The contribution due to $S_{h_{0}}^{2}$ is given by

$$
\begin{aligned}
\left\langle S_{h_{0}}^{2}\right\rangle & =h_{0}^{2} \int_{0}^{\beta} d \tau d \tau^{\prime} \sum_{\substack{\mathbf{r}, \mathbf{r}^{\prime} \\
\nu, \nu^{\prime}=x, y, z}} B_{0 \mathbf{r}}^{\nu}(\tau) \Pi_{00}^{\nu \nu^{\prime}}\left(\mathbf{r}, \tau ; \mathbf{r}^{\prime}, \tau^{\prime}\right) B_{0 \mathbf{r}^{\prime}}^{\nu^{\prime}}\left(\tau^{\prime}\right) \\
& =h_{0}^{2} \sum_{\substack{\tilde{q}, \tilde{q}^{\prime} \\
\nu, \nu^{\prime}=x, y, z}} B_{0}^{\nu}(-\tilde{q}) \Pi_{00}^{\nu \nu^{\prime}}\left(\tilde{q}, \tilde{q}^{\prime}\right) B_{0}^{\nu^{\prime}}\left(\tilde{q}^{\prime}\right) .
\end{aligned}
$$

Since $B_{0 \mathbf{r}}^{\nu}$ is slowly varying, we can evaluate $\Pi_{00}^{\nu \nu^{\prime}}\left(\tilde{q}, \tilde{q}^{\prime}\right)$ at $\tilde{q}=\tilde{q}^{\prime}=0$ :

$$
\left\langle S_{h_{0}}^{2}\right\rangle=h_{0}^{2} \Pi_{00}^{x x} \int_{0}^{\beta} d \tau \sum_{\mathbf{r}} \sin ^{2} \theta_{\mathbf{r}}
$$

Here and in the following, we use the notation $\Pi_{00}^{\nu \nu^{\prime}} \equiv \Pi_{00}^{\nu \nu^{\prime}}\left(\tilde{q}=0, \tilde{q}^{\prime}=0\right)$. We have used $\Pi_{00}^{\nu \nu^{\prime}} \propto \delta_{\nu, \nu^{\prime}}\left(\delta_{\nu, x}+\delta_{\nu, y}\right)$. A similar calculation gives

$$
\begin{aligned}
\left\langle S_{h_{0}} S_{p}\right\rangle & =h_{0} \Pi_{00}^{x x} \int_{0}^{\beta} d \tau \sum_{\mathbf{r}} \frac{i}{2} \sin ^{2} \theta_{\mathbf{r}} \dot{\varphi}_{\mathbf{r}}, \\
\left\langle S_{h_{0}} S_{l}\right\rangle & =h_{0} m_{0} \Pi_{00}^{x x} \int_{0}^{\beta} d \tau \sum_{\mathbf{r}} \sin \theta_{\mathbf{r}}\left(-\cos \psi_{\mathbf{r}} l_{\mathbf{r}}^{x}+\sin \psi_{\mathbf{r}} l_{\mathbf{r}}^{y}\right) .
\end{aligned}
$$

To express $\left\langle S_{h_{0}}^{2}\right\rangle,\left\langle S_{h_{0}} S_{p}\right\rangle$ and $\left\langle S_{h_{0}} S_{l}\right\rangle$ in terms of $\mathbf{n}$ and $\mathbf{l}$, we use

$$
\begin{aligned}
\left(\mathbf{h}_{0} \times \mathbf{n}_{\mathbf{r}}\right)^{2} & =h_{0}^{2} \sin ^{2} \theta_{\mathbf{r}}, \\
\mathbf{h}_{0} \cdot\left(\mathbf{n}_{\mathbf{r}} \times \dot{\mathbf{n}}_{\mathbf{r}}\right) & =h_{0}^{2} \dot{\varphi}_{\mathbf{r}} \sin ^{2} \theta_{\mathbf{r}}, \\
L_{\mathbf{r}}^{z} & =\sin \theta_{\mathbf{r}}\left(-\cos \psi_{\mathbf{r}} l_{\mathbf{r}}^{x}+\sin \psi_{\mathbf{r}} l_{\mathbf{r}}^{y}\right) .
\end{aligned}
$$

The last result follows from $\mathbf{L}_{\mathbf{r}}=\mathcal{R}_{\mathbf{r}} \mathbf{l}_{\mathbf{r}}$ and $l_{\mathbf{r}}^{z}=0$, where $\mathcal{R}_{\mathbf{r}}$ is given by

$$
\mathcal{R}_{\mathbf{r}}=\left(\begin{array}{ccc}
\cos \theta_{\mathbf{r}} \cos \varphi_{\mathbf{r}} \cos \psi_{\mathbf{r}}-\sin \varphi_{\mathbf{r}} \sin \psi_{\mathbf{r}} & -\cos \theta_{\mathbf{r}} \cos \varphi_{\mathbf{r}} \sin \psi_{\mathbf{r}}-\sin \varphi_{\mathbf{r}} \cos \psi_{\mathbf{r}} & \sin \theta_{\mathbf{r}} \cos \varphi_{\mathbf{r}} \\
\cos \theta_{\mathbf{r}} \sin \varphi_{\mathbf{r}} \cos \psi_{\mathbf{r}}+\cos \varphi_{\mathbf{r}} \sin \psi_{\mathbf{r}} & -\cos \theta_{\mathbf{r}} \sin \varphi_{\mathbf{r}} \sin \psi_{\mathbf{r}}+\cos \varphi_{\mathbf{r}} \cos \psi_{\mathbf{r}} & \sin \theta_{\mathbf{r}} \sin \varphi_{\mathbf{r}} \\
-\sin \theta_{\mathbf{r}} \cos \psi_{\mathbf{r}} & \sin \theta_{\mathbf{r}} \sin \psi_{\mathbf{r}} & \cos \theta_{\mathbf{r}}
\end{array}\right) .
$$

We therefore obtain

$$
\begin{aligned}
\left\langle S_{h_{0}}^{2}\right\rangle & =\Pi_{00}^{x x} \int_{0}^{\beta} d \tau \sum_{\mathbf{r}}\left(\mathbf{h}_{0} \times \mathbf{n}_{\mathbf{r}}\right)^{2}, \\
\left\langle S_{h_{0}} S_{p}\right\rangle & =\frac{i}{2} \Pi_{00}^{x x} \int_{0}^{\beta} d \tau \sum_{\mathbf{r}} \mathbf{h}_{0} \cdot\left(\mathbf{n}_{\mathbf{r}} \times \dot{\mathbf{n}}_{\mathbf{r}}\right), \\
\left\langle S_{h_{0}} S_{l}\right\rangle & =m_{0} \Pi_{00}^{x x} \int_{0}^{\beta} d \tau \sum_{\mathbf{r}} \mathbf{h}_{0} \cdot \mathbf{L}_{\mathbf{r}} .
\end{aligned}
$$

${ }^{1}$ For a review on phase fluctuations in 2D superconductors, see V.M. Loktev, R.M. Quick, and S.G. Sharapov, Phys. Rep. 349, 1 (2001).

2 See also V.J. Emery and S.A. Kivelson, Nature 374, 434
(1995).

3 For a review on the BCS-Bose crossover in Fermi superfluids, see M. Randeria in Bose-Einstein condensation, edited by A. Griffin, D.W. Snoke, and S. Strinagari (Cambridge 
University Press, 1995).

${ }^{4}$ For earlier works on the BCS-Bose crossover in Fermi superfluids, see D.M. Eagles, Phys. Rev. 186, 456 (1969); A.J. Leggett in Modern Trends in the Theory of Condensed Matter, edited by A. Peralski and J. Przystawa (SpringerVerlag, Berlin, 1980); P. Nozières and S. Schmitt-Rink, J. Low Temp. Phys. 59, 195 (1985).

5 See, for instance, M. Greiner, C.A. Regal, and D.S. Jin, Nature 426, 437 (2003); M.W. Zwierlein, C.A. Stan, C.H. Schunk, S.M.F. Raupach, S. Gupta, Z. Hadzibabic, and W. Ketterle, Phys. Rev. Lett. 91, 250401 (2003); S. Joachim, M. Bartenstein, A. Altmeyer. S. Riedl, C. Chin, J.H. Denschlag, and R. Grimm, Science 302, 2102 (2003); C.A. Regal, M. Greiner, and D.S. Jin, Phys. Rev. Lett. 92, 040403 (2004); J. Kinast, S.L. Hemmer, M.E. Gehm, A. Turpalov, and J.E. Thomas, Phys. Rev. Lett. 92, 150402 (2004); T. Bourdel, L. Khaykovich, J. Cubizolles, J. Zhang, F. Chevy, M. Teichmann, L. Tarruel, S.J.J.M.F Kokkelmans, and C. Salomon, cond-mat/0403091

${ }^{6}$ R.P. Feynman, Statistical Mechanics (Benjamin, 1972).

7 P. Ao, D.J. Thouless, and X.M. Zhu, Mod. Phys. Lett. B 9, 755 (1995).

8 E.P. Gross, Nuovo Cimento 20, 454 (1961).

9 L.P. Pitaevskii, Sov. Phys. JETP 12, 155 (1961).

10 M. Stone, Int. J. Mod. Phys. B 9, 1359 (1995).

11 I.J.R Aitchison, P. Ao, D.J. Thouless, and X.M. Zhu, Phys. Rev. B 51, 6531 (1995).

12 S. De Palo, C. Castellani, C. Di Castro, and B.K. Chakraverty, Phys. Rev. B 60, 564 (1999).

13 M. Drechsler and W. Zwerger, Ann. Physik 1, 15 (1992).

14 C. A. R. Sá de Melo, M. Randeria, and J. R. Engelbrecht, Phys. Rev. Lett. 71, 3202 (1993); J. R. Engelbrecht, M. Randeria, and C. A. R. Sá de Melo, Phys. Rev. B 55, 15153 (1997).

15 F. Pistolesi and G.C. Strinati, Phys. Rev. B 53, 15168 (1996).

16 S. Stintzing and W. Zwerger, Phys. Rev. B 56, 9004 (1997).

17 E. Babaev and H. Kleinert, Phys. Rev. B 59, 12083 (1999).

18 L. Benfatto, A. Toschi, and S. Caprara, Phys. Rev. B 69, 184510 (2004).

19 V.L. Berezinskii, Zh. Eksp. Teor. Fiz. 59, 907 (1970).

20 J. Kosterlitz and D. Thouless, J. Phys. C 6, 1181 (1973).

${ }^{21}$ R.T. Scalettar, E.Y. Loh, J.E. Gubernatis, A. Moreo, S.R. White, D.J. Scalapino, R.L. Sugar, and E. Dagotto, Phys. Rev. Lett. 62, 1407 (1989).

22 A. Moreo and D.J. Scalapino, Phys. Rev. Lett. 66, 946 (1991).

${ }^{23}$ K. Borejsza and N. Dupuis, EuroPhys. Lett. 63, 722 (2003).

${ }^{24}$ K. Borejsza and N. Dupuis, Phys. Rev. B 69, 085119 (2004).

25 N.D. Mermin and H. Wagner, Phys. Rev. Lett. 17, 1133 (1966).

${ }^{26}$ R. Micnas, J. Ranninger, and S. Robaszkiewicz, Rev. Mod. Phys. 62, 113 (1990).

27 Z.Y. Weng, C.S Ting and T.K. Lee, Phys. Rev. B 43, 3790 (1991).

${ }^{28}$ See also H.J. Schulz in The Hubbard Model, edited by D. Baeriswyl (Plenum, New York, 1995).

29 This procedure bears some analogies with the one used in Ref. 12 .

${ }^{30}$ P.W. Anderson, Phys. Rev. 112, 1900 (1958).

31 N.N. Bogoliubov, JETP 34, 41 (1958); 34, 51 (1958);

${ }^{32}$ I.J.R. Aitchison, G. Metikas, and D.J. Lee, Phys. Rev. B
62, 6638 (2000).

33 S.G. Sharapov, H. Beck, and V.M. Loktev, Phys. Rev. B 64, 134519 (2001).

34 Electromagnetic gauge invariance implies that the time component $A_{0}^{\text {el }}$ of the electromagnetic vector potential enters the effective action $S[\rho, \Theta]$ in the covariant derivative term $\left(i \dot{\Theta}_{\mathbf{r}}-2 e A_{0 \mathbf{r}}^{\mathrm{el}}\right)$, where $e$ is the fermion charge. Since $e \rho_{\mathbf{r}}=-\delta S / \delta A_{0 \mathbf{r}}^{\mathrm{el}}$, the action must contain the term $\int_{0}^{\beta} d \tau \int d^{2} r \frac{\rho_{\mathbf{r}}}{2}\left(i \dot{\Theta}_{\mathbf{r}}-2 e A_{0 \mathbf{r}}^{\mathrm{el}}\right)$, which identifies $\rho_{\mathbf{r}} / 2$ as the conjugate variable of $\Theta_{\mathbf{r}}$.

35 It seems easier to determine the coefficients $\Pi_{\rho \rho}, \Pi_{|\Delta||\Delta|}$ and $\Pi_{\rho|\Delta|}$ by working directly in the attractive Hubbard model and introducing Hubbard-Stratonovich fields both in the particle-particle and particle-hole channels as done in Ref. 12. This approach, however, suffers from the difficulties mentioned after Eqs. (7) in Sec. III

${ }^{36}$ See, for instance, N. Nagaosa, Quantum Field Theory in Condensed Matter Physics (Springer, 1999).

37 Here we use $\Pi_{00}^{x x}(\mathbf{Q}, \mathbf{Q}) \simeq-(2 / h) \Pi_{0 x}^{x y}(\mathbf{Q}, \mathbf{Q}) \simeq$ $-\left(2 / h_{0}\right) \Pi_{0 x}^{x y}(\mathbf{Q}, \mathbf{Q})$ in the weak-coupling limit.

38 A. Auerbach, Interacting Electrons and Quantum Magnetism (Springer-Verlag, New York, 1994).

39 For a derivation of the Magnus force acting on a vortex in a fermion superfluid, based on a Berry phase term calculation, see P. Ao and D.J. Thouless, Phys. Rev. Lett. 70, 2158 (1993).

40 L. Belkhir and M. Randeria, Phys. Rev. B 45, 5087 (1992).

41 L. Belkhir and M. Randeria, Phys. Rev. B 49, 6829 (1994).

42 T. Kostyrko and R. Micnas, Phys. Rev. B 46, 11025 (1992).

43 P.J.H. Denteneer, G. An, and J.M.J. van Leeuwen, EuroPhys. Lett. 16, 5 (1991).

44 P.J.H. Denteneer, G. An, and J.M.J. van Leeuwen, Phys. Rev. B 47, 6256 (1993).

45 J.M. Singer, T. Schneider, and M.H. Pedersen, Eur. Phys. J. B 2, 17 (1998).

46 J.J. Vicente Alvarez and C.A. Balseiro, Solid State Com. 98, 313 (1996).

47 A. Paramekanti, M. Randeria, T.V. Ramakrishnan, and S.S. Mandal, Phys. Rev. B 62, 6786 (2000).

48 J. Tobochnik and G.V. Chester, Phys. Rev. B 20, 3761 (1979).

49 J.F. Fernandez, M.F. Ferreira, and J. Stankiewicz, Phys. Rev. B 34, 292 (1986).

${ }^{50}$ R. Gupta, J. DeLapp, G.G. Batrouni, G.C. Fox, C.F. Baillie, and J. Apostolakis, Phys. Rev. Lett. 61, 1996 (1988).

51 B. Kyung, S. Allen, and A.M.-S. Tremblay, Phys. Rev. B 64, 075116 (2001).

${ }^{52}$ Y.M. Vilk and A.-M.S. Tremblay, J. Phys. I (France) 7, 1309 (1997).

53 J.M. Singer, T. Schneider, and P.F. Meier, Eur. Phys. J. B 7, 37 (1999).

${ }^{54}$ S. Allen, H. Touchette, S. Moukouri, Y.M. Vilk, and A.M.S. Tremblay, Phys. Rev. Lett. 83, 4128 (1999).

55 V.P. Gusynin, V.M. Loktev, and S.G. Sharapov, JETP 90, 993 (2000).

${ }^{56}$ V.P. Gusynin, V.M. Loktev, R.M. Quick, and S.G. Sharapov, Physica C 370, 239 (2002).

57 Note that the (self-consistent) $T$-matrix approximation fails to reproduce the pseudogap in the spectral function $A(\mathbf{k}, \omega)$. For a critical discussion and references see Ref. 51 .

58 S. Sachdev, Z. Physik B 94, 469 (1994).

59 S. Sachdev, Quantum Phase Transitions (Cambridge University, Cambridge, England, 1999). 
60 Note that the $m$ field in Sec. III plays the role of the $m^{\text {HS }}$ field introduced in Sec. II] See the discussion after Eq. [10]).

61 F.D.M. Haldane, Phys. Lett. 93A, 464 (1983).

${ }^{62}$ Note that in Ref. 24, the contribution $S_{\delta m}$ due to amplitude fluctuations was not considered. It is well known that for $h_{0}=0$, amplitude and direction fluctuations decouple in the long wavelength limit, as follows from $\left\langle S_{p} S_{\delta m}\right\rangle=0$ and $\left\langle S_{l} S_{\delta m}\right\rangle=0$ (Appendix E).

${ }^{63}$ It is instructive to write the ferromagnetic magnetization in the general case as a function of $\mathbf{n}_{\mathbf{r}}$ and $\mathbf{L}_{\mathbf{r}}$ using the action (74): $\left\langle c_{\mathbf{r}}^{\dagger} \sigma^{z} c_{\mathbf{r}}\right\rangle=(N \beta)^{-1} \partial_{h_{0}} \ln Z=\Pi_{00}^{x x}\left[m_{0}\left\langle L_{\mathbf{r}}^{z}\right\rangle+\right.$ $\left.\frac{i}{2}\left\langle\hat{\mathbf{z}} \cdot\left(\mathbf{n}_{\mathbf{r}} \times \dot{\mathbf{n}}_{\mathbf{r}}\right)\right\rangle+h_{0}\left\langle\left(\hat{\mathbf{z}} \times \mathbf{n}_{\mathbf{r}}\right)^{2}\right\rangle\right]$. In the strong-coupling limit, $m_{0}=U / 2$ and $\Pi_{00}^{x x}=2 / U$ so that $\left\langle c_{\mathbf{r}}^{\dagger} \sigma^{z} c_{\mathbf{r}}\right\rangle=\left\langle L_{\mathbf{r}}^{z}\right\rangle$ to leading order in $1 / U$, which is the result obtained in the Heisenberg model ${ }^{59}$ In the weak-coupling limit, $m_{0} \sim$ $t e^{-2 \pi \sqrt{t / U}}$ and $\Pi_{00}^{x x} \sim t^{-1}(t / U)^{1 / 2}$. The main contribution to $\left\langle c_{\mathbf{r}}^{\dagger} \sigma^{z} c_{\mathbf{r}}\right\rangle$ comes from the last two terms in the preceding expression of $\left\langle c_{\mathbf{r}}^{\dagger} \sigma^{z} c_{\mathbf{r}}\right\rangle$.

${ }^{64}$ S. Chakravarty, B.I.Halperin, and D.R. Nelson, Phys. Rev. B 39, 2344 (1989).

65 D.S. Fisher, Phys. Rev. B 39, 11783 (1989).

66 The mapping onto the Heisenberg model and the collective mode energy [Eq. (95)] have also been obtained in Ref. 42.

67 The effect of charge fluctuations at strong-coupling near half-filling has also been discussed by L. Benfatto, A. Toschi, S. Caprara, and C. Castellani, Phys. Rev. B 66, 054515 (2002).

68 P. Pieri and G.C. Strinati, Phys. Rev. Lett. 91, 030401 (2003).

69 G.C. Strinati and P. Pieri, Phys. Rev. A 69, 011601(R) (2004). 